Universidade de São Paulo

Instituto de Física e Química de São Carios

\title{
Instrumentação para \\ Implementação da Técnica \\ de Rotação da Amostra em \\ Torno do Ângulo Mágico para \\ Espectroscopia de Sólidos por $\mathrm{RMN}$
}

Gilberto Moretto

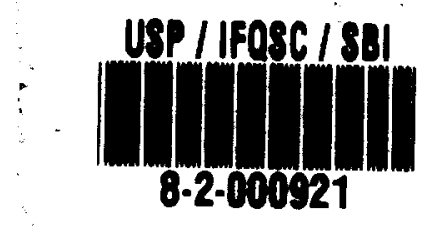

Dissertaçào apresentada ao Instituto de Física e Química de São Carios para obtençāo do título de Hestre em Física Aplicada.

Orientador: Prof.Dr. Horacıo Carios Panepucci.

Departamento de Física e Ciência dos Materiais

Sāo Carlos - 1991 
Esse texto foi preparado

com $\operatorname{IAT}_{E} X$ Version 2.09 .

Lamport, Leslie.

${ }^{A} T_{\mathrm{E}} \mathrm{X}$ : A Document Preparation System.

Special version of Donald Knuth's $T_{E} X$ program. 
MFARPOE CA COMISSAO ULGGAORA DA DISSERTAGAO DE MESTRADO DE GILBEPTO MORETTO APRESENTADÁ AO IMSTITIITO DF FISICA E OUIMTCA DE SAO CARLOS, DA UNIVERSIDADE DE SAO PAULO, EM 25.04.91

COMISSAO JULGHOORA:

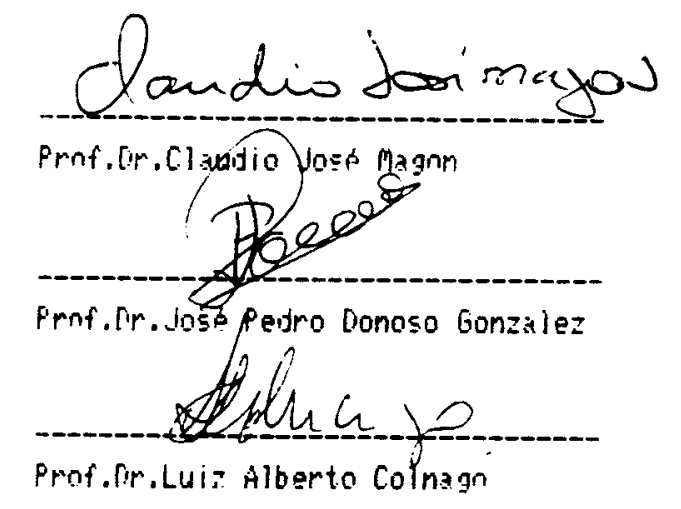


Dominus Illuminatio Mea 
$A$ meus pais, Orlando Moretto Antonia Breschiglicivo Moretto e a amiga e companheira Bartira Mendes Soares pelo carinho e fror tudo mais. 

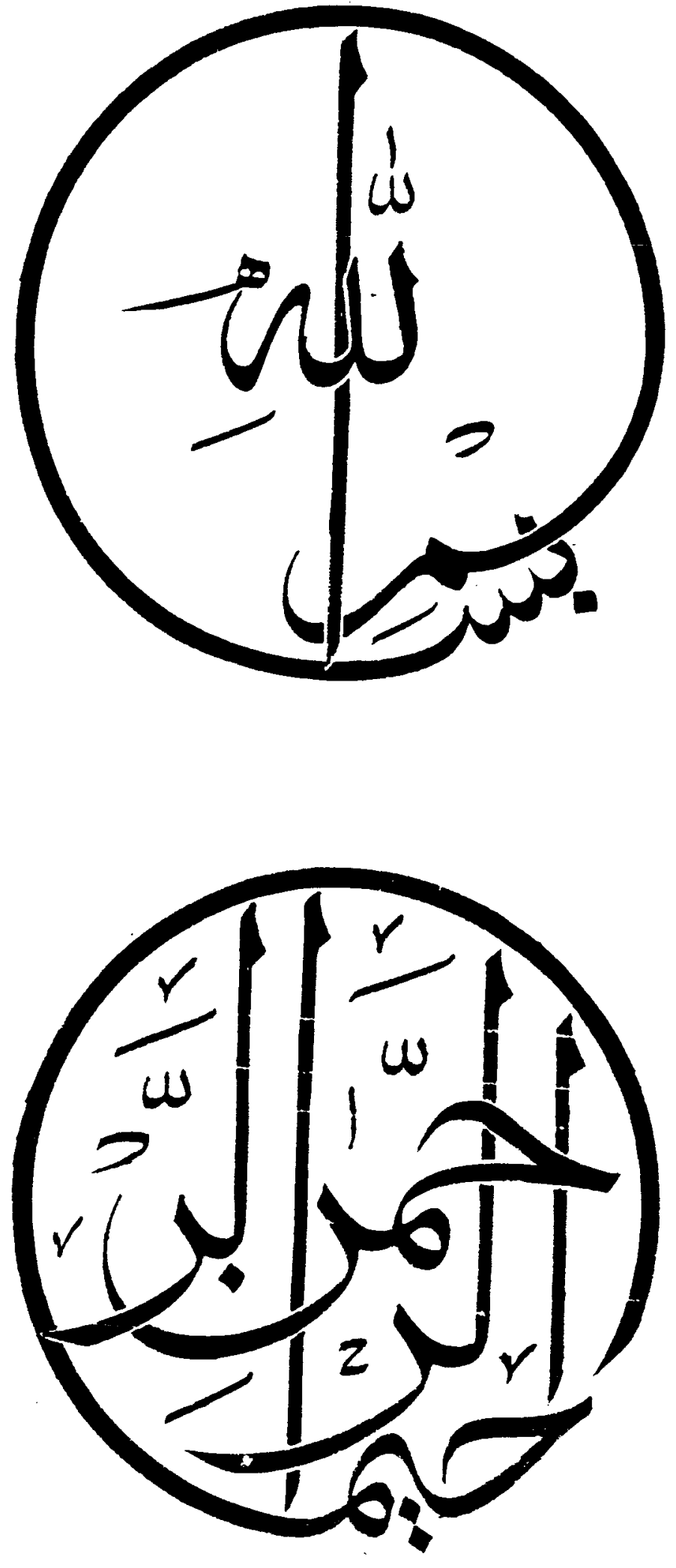


\section{Agradecimentos}

Gostaria, aqui, de manifestar meus sinceros agradecimentos a todas as pessoas que contribuiram para a realização desse trabalho e tornaram-no possível e agradável.

Prof. Tito J. Bonagamba e Prof. Dr. Horácio C. Panepucci pela orientação e discussões tấo frutíferas.

Prof. Dr. Claudio J. Magon que se dispos a substituir o Prof. Panepucci, a partir de dezembro de 1990, devido a sua viagem aos EUA.

Prof. Dr. Jarbas C. Castro Neto pela amizade, apoio, incentivo e preocupação com o futuro papel do Físico no Brasil.

Prof. Dr. Luis Dias da Fac. de Farmácia e Bioquímica da Universidade de Buenos Aires, que nos iniciou em sistema de rotação com mancais aerostáticos cilíndricos.

Engenheiro do grupo Edson L. G. Vidoto pelo auxílio técnico nesse projeto, sempre com carinho e paciência, característicos de sua pessoa.

Dr. Luiz A. Colnago da Embrapa pelo apoio, discussóes e pelas amostras.

Engenheiro André T. Neto da Embrapa pelo apoio e discussões.

Prof. Dr. Henrique Cunha Jr., (EESC-USP), pela orientação em robótica no início desse programa de mestrado; infelizmente, tal projeto não foi possível.

Técnicos do laboratório de Ressonância Magnética, Odir A. Canevarollo, João G. Silva Filho, José Menegazzo e Carlos Gazziro pelas discussóes técnicas tão importantes para a construção de Rotores e Cabeçotes de RMN.

Pessoal da Oficina Mecânica do DFCM, Celso E. Ferri, Manoel R. Roncon, Carlos N. Gonçalves, José R. Pelissari, Marcos J. Estefani e João C. Santos, pela confeç̧ão e sugestões tão frutíferas ao nosso sistema dé rotação e cabeçotes de $R M N$.

Bartira M. Soares e Jane Bonagamba pelas correções ortográficas.

Isabel C. V. Sertori, secretária do Grupo de Ótica, pela paciência, carinho que nos dedica no cumprimento de sua profissão.

Pessoal do Grupo de Ressonância, Telmo L. Euler, Maria Gorette Cavalcante, Valmir Fadel, Pedro Frare, Peter Krauss, Ricardo Giannoni, pela amizade $e$ companhia nesse trabalho.

Claudio Vincenzi, Prof. Dr. Jan F.W. Slaets, Prof. Dr. Carlos A. Ruggiero ("Toto"), Fredy J. Valente, Paulo Ruffino, Valdir C. Melchior, pelo apoio e esclarecimentos junto ao VAX 6420 do IFQSC-USP e do $\mathrm{IAT}_{\mathrm{E}} \mathrm{X}$. 
Italo Celestini e Eduardo P. Freitas, pela prontidão, e dedicação a nossos infindáveis trabalhos de gráfica e a "Tides" C. Vieira, pelas ... cópias xerox.

Pessoal da vigia, Paulo S. dos Santos, Silvio Aguiar, João A. Dotta, pela companhia de noites \& madrugadas adentro.

Dna. Ivoni Clemente, pelos, já tradicionais chás, cafés e sucos sempre dedicados a um dia mais agradável.

\section{Aos amigos}

Tai, sentimento indispensável para a harmonia do ser humano. Bem, alguns foram citados acima, outros agora virão.

"Caeco" Lutz, "Lu" Vencato, Katia Assolan, Carlos Godo, "Edu" Varjão, "Tuto" Lutz ... e todos mais que partilham do caminho.

Alexandre Martinez (Grenoble), Tomaz Catunda (Boston), Luis A. Cury (Toulouse), Marcelo Maialle (S. Diego), Valmor Mastellaro (Orsay), Tião J. N. Pádua (Stony Brook), Clisthenis P. Constantinidis \& Tera (S.Paulo), Marcos Manzocchi (Nice), Luis A. O. Nunes (Red Bank), J. Leonil Duarte (Londrina), todos agora longe, porém, perto em minhas lembranças.

Henrique Barcellos ${ }^{1}$ \& Karla, Almir A. Massambani, Peter William, "Paulinho" Andrade \& "Ro" Ghidelli, pelas discussões sobre a vida noites \& madrugadas adentro.

Cesar Oliveira, Reginaldo J. Napolitano \& Claudia Guaratini, Esmerindo "Gandhi" \& Fatima Sousa, Miled H. Y. Moussa, Wilson B. da Costa, Pedro Del Santoro, Nagib M. Hazbun, Fátima M. Yasuoka, Adão A. Silva, Newton La Scala Jr., Fernando Delboni, Braz Bello Jr., Dorotéia F. Bozano, Mauro Masili, Claudio Lima ..., pelos dia a dia tão agradáveis.

"Pessoar lá di casa", Ivo "Maionese" Vieira \& Harumi Nakahara, Nelson \& Bete Sato, "Lili" Tonetti, "Ge" B. Romanelli, Marco "Mangüeba" Brandão, Silvete M. Soares \& Marcos Chinellato, Dalton Pessoa e aos três pequenos.

Ao Amadeu pela arte de fazer um bom lanche, satisfazendo nosso apetite madrugadas adentro.

Bem, devo ter esquecido alguém. Por favor, mil desculpas.

\footnotetext{
${ }^{1}$ As coisas não são bem assim do jeito que nós pensamos que elas são mesmo!
} 


\section{Sumário}

Este trabalho experimental teve como objetivo o desenvolvimento instrumental necessário para implementação da técnica de Rotação em torno do Ângulo Mágico (MAS). Tal técnica tem por objetivo anular os efeitos das interações dipolares, bem como a interação de deslocamento químico anisotrópico, que provocam o alargamento das linhas de RMN em sólidos. Essas interações anisotrópicas possuem dependências geométricas do tipo $\left(3 \cos ^{2} \theta-1\right)$, sendo $\theta$ o ângulo entre o campo magnético externo $B_{0}$ e o eixo principal do Sistema de Eixos Principais (SEP). O efeito dessas interações podem ser canceladas através de um ajuste fino do ângulo de rotação da amostra com respeito a direção do campo magnético externo, quando a rotação da amostra for maior que a frequência tipica da interação. Isto acontece num ângulo de $54^{\circ} 44^{\prime}$, dito "ângulo mágico"

O cabeçote de RMN, que é composto de um ressonador e um sitema de alta rotação é descrito em detalhes. $O$ desenvolvimento de sistemas de rotação com mancais de ar cilindrico que obtemos pode atingir até $1 \mathrm{KHz}$ sendo usado nesse trabalho para o estudo de nucleos de $C^{13}$ e $H^{1}$.

Para avaliarmos a perfomance do nosso espectrometro apresentamos resultados experimentais obtidos com semente de soja $\left(H^{1}\right)$ e adamantano $\left(C^{13}\right)$. 


\begin{abstract}
The main goal of this work is to develop the necessary instrumentation for the Magic Angle Spinning (MAS) technique, which can be used to remove the dipolar broadening of the NMR spectra. This interaction and also the anysotropic Chemical Shift, have the angular dependence of the type $\left(3 \cos ^{2} \theta-1\right)$, when $\theta$ is the angle between the magnetic external field $\mathrm{B}_{o}$ and the principal axes of Principal Axis Frame. The effect of all these interactions can be canceled by a fine adjustment of the rotation angle of the sample with respect to the magnetic external field $\mathbf{B}_{o}$ direction when the spinning frame is higher than the typical interaction frequency. This happen at an angle of $54^{\circ} 44^{\prime}$ the so called "Magic Angle".

The NMR probe, which is composed by the resonator and the high speed spining system is described in detail. Developed to work with cilindrical air berings the rotation system can achieved speed of $1 \mathrm{KHz}$ and can used to study of nucleus such as $H^{1}, C^{13}$.

To evaluate the perfomance of the spectrometer is presented the experimental results obtained in soybean $\left(H^{1}\right)$ and adamantane $\left(C^{13}\right)$.
\end{abstract}




\section{Conteúdo}

$\begin{array}{ll}\text { Introdução } & 1\end{array}$

1 Hamiltoniano de Spin Nuclear em Sólidos 5

1.1 Introdução . . . . . . . . . . . . . . . . . 5

1.2 Rotações nos Hamiltonianos de Spin Nuclear . . . . . . . . . . . . . . 6

1.3 Hamiltoniano Dipolar ................... 8

1.3.1 Sistema de dois núcleos com $\left(\gamma_{1} \neq \gamma_{2}\right) \ldots \ldots \ldots 12$

1.3.2 Sistema de dois núcleos com $\gamma_{1}=\gamma_{2} \ldots \ldots \ldots \ldots 14$

1.3.3 Significado do Alfabeto Dipolar .............. . 18

1.4 Deslocamento Químico em Sólidos . . . . . . . . . . . . . . . 24

1.4.1 Introdução . . . . . . . . . . . . . . . 24

1.4.2 Interação de Deslocamento Químico . . . . . . . . . . . 25

1.5 Espectros de Pó . . . . . . . . . . . . . . . . . . 32

1.5.1 Formas de Linhas . . . . . . . . . . . . . . . . . . 32

1.5.2 Acoplamento Dipolar de um Sistema AX . . . . . . . . . 36

2 Rotação da amostra em Torno do Ângulo Mágico $4 \mathbf{4 1}$

2.1 Introdução . . . . . . . . . . . . . . . . . . . . 41

2.2 Rotaçōes e Sistemas de Referências . . . . . . . . . . . . . . . . 42

2.3 Ecos Rotacionais e Bandas Laterais . . . . . . . . . . . . . . . . . 44

3 Instrumentacão Básica $\quad \mathbf{5 5}$

3.1 Introdução . . . . . . . . . . . . . . . . . . . 55

3.2 O Magneto ........................ 57

3.3 Fonte de Radiação . . . . . . . . . . . . . . . . . . . 57

3.4 Recepção do Sinal . . . . . . . . . . . . . . . . . . . . . . 58

3.5 Sistema de Controle . . . . . . . . . . . . . . . . . . 58

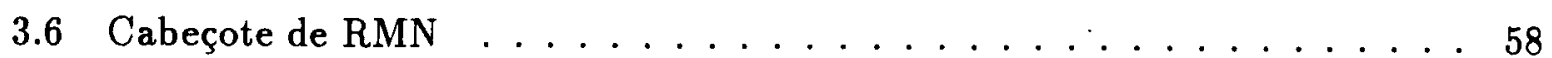

3.7 Cálculos para a Sonda de RF . . . . . . . . . . . . . . 59

3.8 Montagem da Sonda de RMN ................... 62 
4 Sistema de Rotação da Amostra 69

4.1 Introdução ......................... 69

4.2 Mancais Aerostáticos Radiais ................. 70

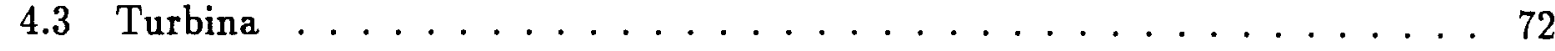

4.4 Seleção dos Materiais . . . . . . . . . . . . . . . . 73

4.5 Nosso Rotor . . . . . . . . . . . . . . . . . 75

5 Resultados Experimentais - $\quad \mathbf{8 3}$

5.1 Apresentação ... . . . . . . . . . . . . . . . 83

5.2 Bandas Laterais \& Ecos Rotacionais . . . . . . . . . . . . . . 83

5.3 Desacoplamento em Alta Potência \& RAAM . . . . . . . . . . . . 84

$\begin{array}{ll}\text { Conclusão } & 91\end{array}$

A Rotações $\quad 03$

A.1 Introdução . . . . . . . . . . . . . . . . . 93

A.2 Matriz dos Operadores Rotação . . . . . . . . . . . . . 94

A.3 Determinação das Matrizes de Rotação . . . . . . . . . . . . . . 96

B Tensores Irredutíveis 103

B.1 Introdução . . . . . . . . . . . . . . . . . . 103

B.2 Componentes de um Tensor Irredutível $\ell=2 \ldots \ldots$. . . . . . . . 104

C Projeto do Sistema de Rotação da Amostra $\quad 109$ 


\section{Introdução}

Nesse trabalho experimental enfocamos a espectroscopia de alta resolução em sólidos por RMN, utilizando amostras com núcleos de spin 1/2. Devido ao fato de amostras sólidas possuirem alta rigidez interna e um estado molecular congelado, resulta em sinais muito largos, da ordem de vários $\mathrm{kHz}$, que impossibilitam a observação de espectros isotrópicos.

Estes alargamentos de sinais provêm de interaçōes anisotrópicas que os vários núcleos de um sólido experimentam com suas vizinhanças. As interações que mais contribuem para tais alargamentos são as interações dipolares (homo e heteronucleares) e o fenômeno de deslocamento químico anisotrópico.

Nossa intenção é o estreitamento controlado dessas linhas espectrais, resultando num espectro isotrópico de alta resolução em sólidos que permita a obtenção de espectros que contenha informaçōes sobre a estrutura química da molécula, isto é, aquele no qual o deslocamento químico isotrópico seja a única interação relevante. Cabe aqui deixar claro que o alargamento do espectro traz também informações importantes porém essas são relevantes á própria interação.

Questionamentos sobre esse estreitamento de linhas espectrais, nos levam a espectros de amostras líquidas, que são muito mais estreitos quando comparados com espectros de amostras sólidas. Esse estreitamento em amostras líquidas devem-se aos movimentos rápidos, aleatórios e isotrópicos de reorientação e translação existentes intrinsecamente numa amostra líquida.

Um outro fator que se deve levar em conta é que, tanto a interação dipolar como o deslocamento químico anisotrópico possuem uma dependência geométrica do tipo $\left(3 \cos ^{2} \theta-1\right)$, onde $\theta$ é o ângulo entre o campo magnético externo $B_{0}$ e o eixo principal do sistema de eixos principais (SEP).

Considerando que os movimentos rápidos, aleatórios e isotrópicos em líquidos resultam em linhas de alta resolução, fica evidente o interesse em introduzí-los artificialmente em amostras sólidas, [1]. Para tal utilizamo-nos da técnica de rotação da amostra em torno de um determinado ângulo $\theta$ - dito ângulo mágico - que anula o termo $\left(3 \cos ^{2} \theta-1\right)$, e consequentemente cancelando os efeitos da interação dipolar e deslocamento químico anisotrópico.

Daí a motivação para o nosso trabalho experimental, onde nos preocupamos com o desenvolvimento do instrumental necessário para implementação de tal técnica, que consiste em prover à amostra uma rotação macroscópica em torno do ângulo dito mágico. Construímos, assim, cabeçotes de RMN que permitem à amostra tais rotaçōes e numa 
frequêencia suficiente para uma eliminação eficiente dos alargamentos de linhas, [3] - [5].

Nessa tese apresentamos no capítulo 1, um estudo das interaçōes anisotrópicas e como elas se manifestam nos espectros, através do formalismo de tensores irredutíveis esféricos, apêndice $A$ \& $B$.

No capítulo 2 estudamos o comportamento dessas interaçōes sob rotação e seus efeitos sobre os espectros. Por sua vez no capítulo 3 discutimos o equipamento básico necessário e no capítulo 4 a elaboração de rotores desenvolvidos em nosso laboratório para rotação da amostra. Finalmente, no capítulo 5 apresentamos resultados obtidos com o nosso rotor.

A elaborada exposição

de desenhos à nanquim, deve-se

ao esmero, paciência \&f talento de

Samuel Alvarez - Desenhista do DFCM

ao qual manifesto meus agradecimentos. 


\section{Bibliografia}

[1] E. R. Andrew, in Philosophical Transactions of The Royal Society of Lc ndon - Mathematical and Physical Sciences - Nuclear Magnetic Resonance Spectroscopy in Solids, 299, 505, No 1452, (1981).

[2] I. I. Lowe, in Phys. Rev. Lett., 2, 285, (1959).

[3] J. S. Waugh, M. M. Maricq, and R. Cantor, in J. Magn. Reson., 29, 183, (1978).

[4] E. O. Stejskal, J. Schaefer, R. A. McKay, in J. Magn. Reson., 25, 256, (1977).

[5] M. M. Mariq and J. S. Waugh, in J. Chem. Phys., 70, 3300 (1979). 
BIBLIOGRAFIA 


\section{Capítulo 1}

\section{Hamiltoniano de Spin Nuclear em Sólidos}

\subsection{Introdução}

Quando nos propomos a analisar interaçōes de spins nucleares em sólidos nos defrontamos com alta rigidez interna e um estado molecular congelado, que resultam em sinais muito largos, da ordem de vários $\mathrm{KHz}$. Estes alargamentos de sinais provêm de interações anisotrópicas ${ }^{1}$ que os vários núcleos de um sólido experimentam com as suas vizinhanças.

Em amostras sólidas de spin nuclear $\frac{1}{2}$, as interação que mais contribuem para o alargamento de espectro de RMN são as interações dipolares, $\mathcal{H}_{D}$ E deslocamento químico anisotrópico, $\mathcal{H}_{D Q}$.

$$
\mathcal{H}_{D Q}=\sum_{i} \gamma_{n}^{i} \mathbf{I}^{i} \cdot \sigma^{i} \cdot \mathbf{B}_{o}
$$

que descreve a interação dos spins nucleares com o campo magnético induzido, devido ao movimento orbital dos elétrons. O tensor $\sigma^{i}$ é o tensor desvio químico anisotrópico no sítio do i-ésimo núcleo e $\gamma_{n}$ é a constante giromagnética, característica do núcleo.

$$
\begin{aligned}
\mathcal{H}_{D} & =\sum_{i<k}\left(-\frac{\gamma_{i} \gamma_{k} \hbar}{r_{i k}^{3}}\right)\left[\frac{3\left(\mathbf{I}^{i} \cdot \mathbf{r}_{i k}\right)\left(\mathbf{I}^{k} \cdot \mathbf{r}_{i k}\right)}{r_{i k}^{3}}-\mathbf{I}^{i} \cdot \mathrm{I}^{k}\right] \\
& =\sum_{i<k}\left(-2 \gamma_{n}^{i} \gamma_{n}^{k} \hbar^{2}\right) \sum_{\alpha, \beta=1}^{3} \mathbf{I}_{\alpha}^{i} \cdot \mathbf{R}_{\alpha \beta}^{i k} \cdot \mathbf{I}_{\beta}^{k}
\end{aligned}
$$

que descreve a interação de spins nucleares entre si, através de seu momento de dipolo magnético, sendo $\vec{r}_{i k}$ o vetor internuclear, vide figura (1.1) e $\mathbf{R}_{\alpha \beta}^{i k}$ componentes do tensor $\mathbf{R}^{i k}$ que são justamente os coeficientes que advem das variaveis geométricas.

\footnotetext{
${ }^{1}$ Dependem da orientação dos eixos cristalográficos relativamente ao campo magnético externo.
} 
Por isso, nesse capítulo iremos estudar essas duas interações, verificando como elas se manifestam sobre os espectros de RMN.

\subsection{Rotações nos Hamiltonianos de Spin Nuclear}

Introduziremos aqui uma estrutura em comum, dada por Haeberlen [2], para expressar os Hamiltonianos de spin nuclear. Isto.é feito em termos de componentes de operadores esféricos irredutíveis, segundo bases de harmônicos esféricos (apêndices A \& B), pois quando a amostra estiver em rotação, esse formalismo se mostrará mais conveniente.

Segundo Haeberlen [2], se desprezarmos a soma sobre os núcleos $i$ ou pares $i, k$ os Hamiltonianos de spin nuclear podem ser expressos por:

$$
\mathcal{H}_{\lambda}=C^{\lambda} \sum_{\alpha, \beta=1}^{3} R_{\alpha, \beta}^{\lambda} T_{\beta \alpha}^{\lambda}
$$

sendo $C^{\lambda}$ uma constante que depende das propriedades nucleares, tais como:

- Interação Dipolar $\rightarrow C^{D}=-2 \gamma_{n}^{i} \gamma_{n}^{k} \hbar$

- Deslocamento Químico $\rightarrow C^{D Q}=\gamma_{n}^{i}$

Os termos $T_{\beta \alpha}^{\lambda}=I_{\alpha} A_{\beta}^{\lambda}$ são produtos de diádicas construídas de dois vetores, onde I é sempre um vetor de spin nuclear, enquanto $\mathrm{A}^{\lambda}$ pode ser um outro spin nuclear, no caso de $\lambda=D$ ou o campo magnético externo, no caso de $\lambda=D Q$.

Por sua vez, $\mathbf{R}^{\lambda}$ que é função de $\sigma^{i}, \mathbf{R}^{i, k}$, depende do estado eletrônico, da configuração geométrica nuclear, estados vibracionais e rotacionais da molécula.

Observe que essa forma compacta de escrever as interaçōes de spin nuclear traz sempre uma parte dependente das variáveis de spin e outra das coordenadas nucleares (parte geométrica), sendo que ambas transformam-se como um tensor de segunda ordem. De (B.2) e (B.4) vimos que dois tensores irredutíveis de ordem iguais podem ser contraidos resultando num invariante. Para os nossos dois tensores irredutíveis de ordem dois, resulta o invariante:

$$
\mathcal{H}_{\lambda}=C^{\lambda} \sum_{l} \sum_{m=-l}^{(\ell)}(-1)^{m} R_{l,-m}^{\lambda} T_{l m}^{\lambda}
$$

onde $R_{l,-m}^{\lambda}$ deriva de $R_{\alpha \beta}^{\lambda}$ e $T_{l m}^{\lambda}$ deriva de $T_{\alpha \beta}^{\lambda}$.

Todo tensor $\mathbf{R}_{\lambda}$ de segunda ordem pode ser decomposto numa soma de tensores irredutíveis de ordem zero, um e dois (Apêndice B):

$$
\mathbf{R}=\mathbf{R}^{0}+\mathbf{R}^{1}+\mathbf{R}^{2}
$$

sendo a parte isotrópica de $\mathbf{R}^{\lambda}$ dada por $\mathbf{R}^{0}=\frac{1}{3} \operatorname{Tr}(\varrho \mathbf{R})=\varrho R$, onde $\varrho$ é a unidade diádica. 
Considerando-se os tensores das interaçōes como simétricos ${ }^{2}$, dependendo da estrutura eletrônica molecular e/ou da simetria do meio cristalino, existe um sistema de eixos no qual esses tensores são diagonais. Esse sistema é dito Sistema de Eixos Principais (SEP), e tais valores diagonais são denominados valores principais do tensor dado por $R_{a a}, R_{b b}, R_{c c}$, (bases $\hat{\mathbf{a}}, \hat{\mathbf{b}}, \hat{\mathbf{c}}$ ).

Para trabalhar com $\mathbf{R}$ simétrico, juntamos a componente isotrópica $\mathbf{R}^{0}$ mais dois parâmetros $\delta$ e $\eta$, definidos por:

$$
\begin{aligned}
\delta & =R_{c c}-\frac{1}{3} \operatorname{Tr} \mathbf{R}=R_{c c}-R \\
\eta & =\frac{R_{a a}-R_{b b}}{R_{c c}-\frac{1}{3} \operatorname{Tr} \mathbf{R}}=\frac{R_{a a}-R_{b b}}{\delta}
\end{aligned}
$$

resultando no sistema de eixos principais (SEP):

$$
\mathbf{R}(S E P)=\left(\begin{array}{ccc}
R_{a a} & 0 & 0 \\
0 & R_{b b} & 0 \\
0 & 0 & R_{c c}
\end{array}\right)=\varrho R+\delta\left(\begin{array}{ccc}
-\frac{1}{2}(1-\eta) & 0 & 0 \\
0 & -\frac{1}{2}(1+\eta) & 0 \\
0 & 0 & 1
\end{array}\right)
$$

A partir dessas consideraçōes vem de (B.9), que $R_{l m}^{\lambda}$ só será diferente de zero se $l=0$ e $l=2$. Agora se estivermos no (SEP) podemos verificar de (B.19)-(B.24) que somente os elementos para $m=0, \pm 2$ serão diferentes de zero, resultando nesse sistema para as componentes do operador tensor esférico irredutíveis $R_{l m}$, denotadas por $\vartheta_{l m}$ :

$$
\begin{aligned}
\vartheta_{00} & =\frac{1}{3} \operatorname{Tr} \mathbf{R}=R \\
\vartheta_{20} & =\sqrt{\frac{3}{2}}\left(R_{c c}-\frac{1}{3} \operatorname{Tr} R\right)=\sqrt{\frac{3}{2}} \delta \\
\vartheta_{2 \pm 2} & =\frac{1}{2}\left(R_{a a}-R_{b b}\right)=\frac{1}{2} \eta \delta .
\end{aligned}
$$

Quanto ao tensor $T_{\hat{\beta} \gamma}^{\lambda}$, precisamos lembrar que $I_{\alpha}$ e $A_{\beta}^{\lambda}$ estão ligados ao sistema de referência do laboratório (LAB); isso é óbvio para $A_{\beta}^{\lambda}$ que pode ser componente do campo estático ou oscilatório e também é verdade para $I_{\alpha}$ que está ligado às variáveis de spin que são as variáveis dinâmicas num experimento de RMN. É sua evolução temporal que observamos utilizando-se de equipamentos de laboratório e portanto do sistema de referência do laboratório. Devemos assim expressar $R_{l m}^{\lambda}$ no referencial do laboratório.

Vimos no apêndice B que sendo $R_{l m}^{\lambda}$ componente de um operador tensor esférico irredutível podemos expressá-lo no LAB em termos de $\vartheta_{l m}$, e das matrizes de rotação de

\footnotetext{
${ }^{2}$ Veremos à frente que os tensores dipolares (D) possuem traços nulos e são simétricos. Por sua vez os tensores de deslocamento químico $(\sigma)$, possuem as suas componentes isotrópicas, simétrica, de traço nulo e a anti-simétrica de traço nulo, porém somente a parte isotrópica e simétrica de traço nulo é mensurável pelas técnicas espectroscópicas de RMN.
} 
Wigner $\mathcal{D}_{m^{\prime} m}^{(\ell)}\left(\alpha^{\lambda} \beta^{\lambda} \gamma^{\lambda}\right)$ por:

$$
R_{l m}^{\lambda}=\sum_{m^{\prime}} \mathcal{D}_{m^{\prime} m}^{(\ell)}\left(\alpha^{\lambda} \beta^{\lambda} \gamma^{\lambda}\right) \vartheta_{l m}^{\lambda}
$$

onde $\alpha^{\lambda}, \beta^{\lambda}, \gamma^{\lambda}$ são os ângulos de Euler que levam o sistema do laboratório a coincidir com o sistema de eixos principais. Como estamos tratando de tensores de segunda ordem somente iremos precisar da matriz $\mathcal{D}_{m}^{(2)}$, expressa no apêndice B.

\subsection{Hamiltoniano Dipolar}

Estaremos agora interessados em discutir explicitamente $\mathcal{H}_{D}$. A situação é a interação de dois núcleos de spin $\frac{1}{2}$ com momentos de dipolo magnético $\mu_{1}$ e $\mu_{2}$, dados por: $\mu_{1}=\gamma_{n}^{1} \hbar \mathbf{I}^{1}$ e $\mu_{2}=\gamma_{n}^{2} \hbar \mathrm{I}^{2}$, figura (1.1). Os spins nucleares "veem" não somente o campo magnético aplicado mas também os campos dipolares que se originam dos momentos magnéticos nucleares associados com os spins de todos os vizinhos do núcleo. Será então a soma dos vetores de todos os campos magnéticos no sítio de um núcleo que irá determinar onde esse núcleo irá "aparecer" num espectro de RMN. O campo local criado por cada núcleo no sítio do outro núcleo acoplando-os é dado por:

$$
\begin{aligned}
& \frac{4 \pi}{\mu_{o}} \mathbf{B}_{1}(r)=\frac{\gamma_{1}}{r^{5}} \hbar\left[3\left(\mathbf{I}^{1} \cdot \mathbf{r}\right) \mathbf{r}-r^{2} \mathbf{I}^{1}\right] \\
& \frac{4 \pi}{\mu_{o}} \mathbf{B}_{2}(r)=\frac{\gamma_{2}}{r^{5}} \hbar\left[3\left(\mathbf{I}^{2} \cdot \mathbf{r}\right) \mathbf{r}-r^{2} \mathbf{I}^{2}\right]
\end{aligned}
$$

resultando para o Hamiltoniano de interação dipolar entre esses dois núcleos ${ }^{3}$ :

$$
\hbar \mathcal{H}_{D}=\left(-\frac{\gamma_{1} \gamma_{2} \hbar^{2}}{r_{12}^{3}}\right)\left[\frac{3\left(\mathbf{I}^{1} \cdot \mathbf{r}_{12}\right)\left(\mathbf{I}^{2} \cdot \mathbf{r}_{12}\right)}{r_{12}^{2}}-\mathbf{I}^{1} \cdot \mathbf{I}^{2}\right]
$$

onde $\mathbf{r}_{12}$ é o vetor entre os núcleos 1 e 2 . No caso geral, somamos sobre todos os núcleos $i, k$ possíveis, resultando:

$$
\hbar \mathcal{H}_{D}=\sum_{i<k}\left(-\frac{\gamma_{i} \gamma_{k} \hbar^{2}}{r_{i k}^{3}}\right)\left[\frac{3\left(\mathbf{I}^{i} \cdot \mathbf{r}_{i k}\right)\left(\mathbf{I}^{k} \cdot \mathbf{r}_{i k}\right)}{r_{i k}^{3}}-\mathrm{I}^{i} \cdot \mathbf{I}^{k}\right]
$$

Precisamos agora analisar o termo vetorial $\left(\mathbf{I}^{i} \cdot \mathbf{r}_{i k}\right)\left(I^{k} \cdot \mathbf{r}_{i k}\right)$, para tal lembramo-nos da contração de dois tensores cartesianos:

\footnotetext{
${ }^{3}$ Da interação Zeeman $\mathcal{H}_{z}=-\mu \cdot \mathbf{B}$, sendo $\mathbf{B}=\mathbf{B}_{0}+\mathbf{B}_{1}$ no sitio do núcleo 2 e $\mathbf{B}=\mathbf{B}_{0}+\mathbf{B}_{2}$ no sítio do núcleo 1 .
} 


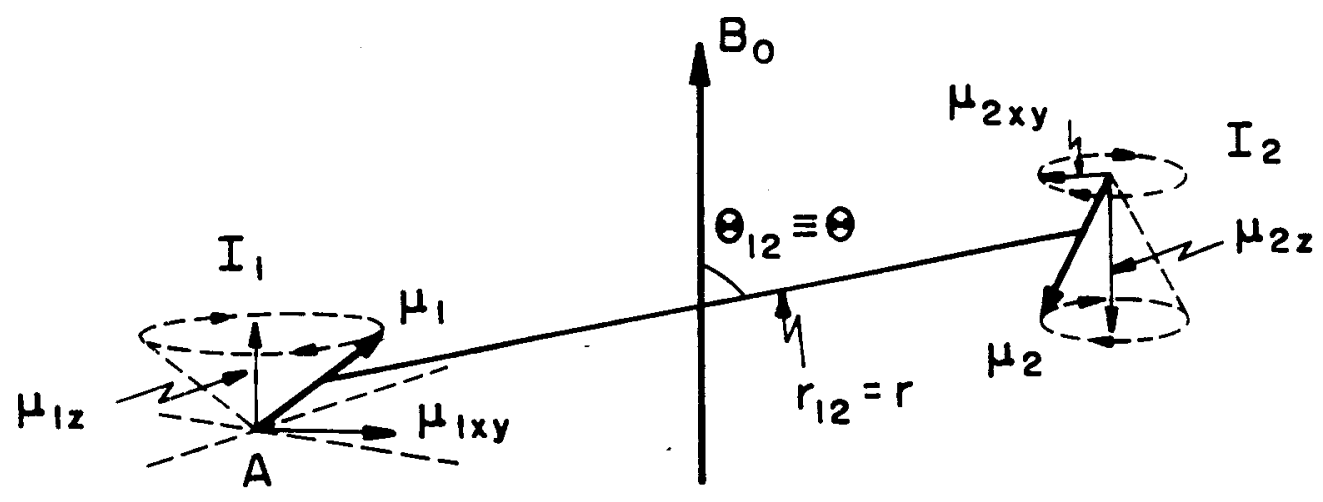

Figura 1.1: Interação Dipolar entre os momentos de dipolo magnético $\mu_{1}$ e $\mu_{2}$ na presença do campo estático $\mathrm{B}_{0}$.

(i) A, B tensores de ordem um, resultando $\mathrm{H}$ invariante dado por:

$$
H=\mathbf{A} \cdot \mathbf{B}=\tilde{\mathbf{A}} \varrho \mathbf{B}=\sum_{\nu=1}^{n} A_{\nu} B_{\nu}
$$

onde $\varrho=\ell \times \ell$ matriz unitária, A é um vetor coluna de dimensão $\ell$ e $\tilde{\mathbf{A}}$ é um vetor linha de dimensão $\ell$.

(ii) $\mathbf{R}, \mathrm{T}$ tensores de ordem dois, resultando $\mathrm{H}$ invariante dado por:

$$
H=\operatorname{Tr}(\tilde{\tilde{\mathbf{R}}} \overline{\tilde{\mathbf{T}}})=\sum_{\nu \epsilon} R_{\nu \epsilon} T_{\epsilon \nu}
$$

Obtemos assim tensores de ordem dois do acoplamento de tensores de ordem um A e $\mathbf{B}$, sendo $\tilde{\tilde{\mathbf{R}}}=\mathbf{A} \tilde{\mathbf{B}}, \tilde{\tilde{\mathbf{T}}}=\mathbf{C} \tilde{\mathbf{D}}$, que resulta:

$$
\begin{aligned}
H & =\operatorname{Tr}(\tilde{\tilde{\mathbf{R}}} \tilde{\tilde{\mathbf{T}}})=\operatorname{Tr}(\mathbf{A} \tilde{\mathbf{B}} \mathbf{C} \tilde{\mathbf{D}})=\sum_{\gamma \lambda}(\mathbf{A} \tilde{\mathbf{B}})_{\gamma \lambda}(\mathbf{C} \tilde{\mathbf{D}})_{\lambda \gamma}=\sum_{\gamma \lambda} A_{\gamma} B_{\lambda} C_{\lambda} D_{\gamma} \\
& =\sum_{\gamma \lambda} D_{\gamma} A_{\gamma} B_{\lambda} C_{\lambda}=\sum_{\gamma \lambda} D_{\gamma}(\mathbf{A} \tilde{\mathbf{B}})_{\gamma \lambda} C_{\lambda}=\tilde{\mathbf{D}}(\mathbf{A} \tilde{\mathbf{B}}) \mathbf{C} \equiv \mathbf{D} \cdot \tilde{\tilde{\mathbf{R}}} \cdot \mathbf{C} \\
& =(\tilde{\mathbf{D}} \mathbf{A})(\tilde{\mathbf{B}} \mathbf{C})=(\mathbf{D} \cdot \mathbf{A})(\mathbf{B} \cdot \mathbf{C})
\end{aligned}
$$

que resulta em várias formas equivalentes de contração de um tensor de ordem dois.

Utilizando-se das relaçōes $(1.18),(1.21)$ e (1.22) resulta para o hamiltoniano dipolar: 


$$
\mathcal{H}_{D}=-2 \gamma_{1} \gamma_{2} \hbar^{2} \mathbf{I}^{1} \cdot \mathbf{R} \cdot \mathbf{I}^{2},
$$

onde $\mathbf{R}=\frac{1}{2 r_{12}^{3}}(3 \hat{\mathbf{r}} \hat{\mathbf{r}}-\varrho)$, é simétrica e de traço nulo, e $\hat{\mathbf{r}}$ é o vetor unitário internuclear no sistema de referência do operador $\mathrm{I}^{1}$ e $\mathrm{I}^{2}$. Para transformar (1.23) na forma de uma contração de tensores esféricos, fazemos $T \equiv \tilde{\mathbf{I}}^{2} \mathbf{I}^{1}$ ou $\mathbf{T} \equiv \mathbf{I}^{1} \tilde{\mathbf{I}}^{2}$ resultando $\mathbf{I}^{1} \cdot \mathbf{R} \cdot \mathbf{I}^{2} \equiv$ $\tilde{\mathbf{I}}^{1} \mathbf{R} \mathbf{I}^{2}=\operatorname{Tr}(\mathbf{R T})$, disso e de (B.4) resulta para o hamiltoniano dipolar:

$$
\begin{aligned}
\mathcal{H}_{D} & =-2 \gamma_{1} \gamma_{2} \hbar^{2} \operatorname{Tr}(\mathbf{R T}) \\
& =-2 \gamma_{1} \gamma_{2} \hbar^{2} \sum_{m}(-1)^{m} R_{2-m} T_{2 m}
\end{aligned}
$$

Retornando para o sistema de eixos principais $(\hat{\mathbf{a}}, \hat{\mathbf{b}}, \hat{\mathbf{c}})$ no sistema cartesiano, colocamos $\hat{\mathbf{r}}_{12}$ sobre $\hat{\mathbf{c}}$, isto é $\left(\hat{\mathbf{r}}_{12}=\hat{\mathbf{c}}\right)$, resultando:

$$
\mathbf{R}=\frac{1}{\mathbf{r}_{12}^{3}}\left(\begin{array}{ccc}
-\frac{1}{2} & 0 & 0 \\
0 & -\frac{1}{2} & 0 \\
0 & 0 & 1
\end{array}\right) .
$$

Conforme (1.7), (1.8), temos $\delta^{D, 12}=\mathbf{r}_{12}^{-3}$ e $\eta^{D, 12}=0$, resultando para as componentes de $\vartheta_{l m}$ :

$$
\begin{aligned}
\vartheta_{00} & =0 \\
\vartheta_{20} & =\sqrt{\frac{3}{2}} r_{12}^{-3}, \\
\vartheta_{2 m^{\prime}} & =0, \text { para } m^{\prime} \neq 0 .
\end{aligned}
$$

Utilizando-se dessas componentes expressamos $R_{l m}$ de (1.13) no LAB, resultando $R_{2 m}=\mathcal{D}_{0 m}^{(2)}\left(\Omega^{D, 12}\right) \sqrt{\frac{3}{2}} r_{12}^{-3}$ e por sua vez para $(1.24)$ :

$$
\mathcal{H}_{D}=-\frac{\sqrt{6} \gamma^{1} \gamma^{2} \hbar^{2}}{r_{12}^{-3}} \sum_{m=-2}^{2}(-1)^{m} \mathcal{D}_{0 m}^{(2)}\left(\Omega^{D, 12}\right) T_{2 m}^{D, 12}
$$

Para as componentes $T_{2 m}^{D, 12}$, lembramos de (B.9), que resulta:

$$
\begin{aligned}
T_{20}^{D, 12} & =\frac{1}{\sqrt{6}}\left[2 U_{10} V_{10}+U_{11} V_{1-1}+U_{1-1} V_{11}\right] \\
T_{2 \pm 1}^{D, 12} & =\frac{1}{\sqrt{2}}\left[U_{1 \pm 1} V_{10}+U_{10} V_{1 \pm 1}\right] \\
T_{2 \pm 2}^{D, 12} & =U_{1 \pm 1} V_{1 \pm 1},
\end{aligned}
$$

onde:

$$
U_{10}=I_{z}^{1}
$$




$$
\begin{gathered}
U_{11}=-\frac{I_{x}^{1}+i I_{y}^{1}}{\sqrt{2}}=-\frac{I_{+}^{1}}{\sqrt{2}}, \\
U_{1-1}=-\frac{I_{x}^{1}-i I_{y}^{1}}{\sqrt{2}}=\frac{I_{-}^{1}}{\sqrt{2}},
\end{gathered}
$$

e da mesma maneira para $\mathrm{V} \equiv \mathrm{I}_{2}$, resultando de (1.30), (1.31) e (1.32):

$$
\begin{aligned}
T_{20}^{D, 12} & =\frac{1}{\sqrt{6}}\left[I_{z}^{1} I_{z}^{2}-\frac{I_{+}^{1} I_{-}^{2}}{2}-\frac{I_{-}^{1} I_{+}^{2}}{2}\right], \\
T_{2 \pm 1}^{D, 12} & =\frac{1}{\sqrt{2}}\left[\mp I_{ \pm}^{1} I_{z}^{2} \mp I_{z}^{1} I_{ \pm}^{2}\right], \\
T_{2 \pm 2}^{D, 12} & =I_{ \pm}^{1} I_{ \pm}^{2} .
\end{aligned}
$$

Quanto aos elementos de $\mathbf{R}(L A B)$, se faz necessário notar que $\beta^{D, i k}$ e $\alpha^{D, i k}$ são respectivamente idênticos aos ângulos polares $\theta^{i k}$ e $\varphi^{i k}$ do vetor internuclear $\mathbf{r}_{i k}$ no referencial do laboratório conforme a figura (1.2). Resulta então para os elementos $R_{2 m}$, (1.13) e utilizando-se dos elementos de Wigner $\mathcal{D}_{0 m}^{(2)}\left(\Omega^{D, 12}\right)$, para tensores de segunda ordem (Apêndice B):

$$
\begin{aligned}
R_{20}(L A B) & =\sqrt{\frac{3}{8}} \delta\left(3 \cos \theta_{12}-1\right) \\
R_{2 \pm 1}(L A B) & = \pm \frac{3}{4} \delta \sin 2 \theta_{12} e^{\mp i \varphi_{12}} \\
R_{2 \pm 2}(L A B) & =\frac{3}{4} \delta \sin ^{2} \theta_{12} e^{\mp i \varphi_{12}} .
\end{aligned}
$$

Voltando ao Hamiltoniano Dipolar, com os elementos $R_{2 m}^{D, 12}$ e $T_{2 m}^{D, 12}$, obtemos $\mathcal{H}_{D}$ na forma de "Alfabeto Dipolar"

$$
\mathcal{H}_{D}=\frac{\gamma_{1} \gamma_{2} \hbar^{2}}{r_{12}^{3}}\{\mathbf{A}+\mathbf{B}+\mathbf{C}+\mathbf{D}+\mathbf{E}+\mathbf{F}\}
$$

onde

$$
\begin{aligned}
& A=\left(\mathbf{I}_{z}^{1} \mathbf{I}_{z}^{2}\right) \times\left(3 \cos ^{2} \theta_{12}-1\right) \\
& B=\left(\mathbf{I}_{+}^{1} \mathbf{I}_{-}^{2}+\mathbf{I}_{-}^{1} \mathbf{I}_{+}^{2}\right) \times \frac{1}{2}\left(3 \cos ^{2} \theta_{12}-1\right) \\
& C=\left(\mathbf{I}_{+}^{1} \mathbf{I}_{z}^{2}+\mathbf{I}_{z}^{1} \mathbf{I}_{+}^{2}\right) \times\left[-\frac{3}{2 \sqrt{2}}\left(\sin 2 \theta_{12} e^{i \varphi_{12}}\right)\right] \\
& D=\left(\mathbf{I}_{-}^{1} \mathbf{I}_{z}^{2}+\mathbf{I}_{z}^{1} \mathbf{I}_{-}^{2}\right) \times \frac{3}{2 \sqrt{2}}\left(\sin 2 \theta_{12} e^{-\varphi_{12}}\right) \\
& E=\left(\mathbf{I}_{+}^{1} \mathbf{I}_{+}^{2}\right) \times \frac{3}{2}\left(\sin ^{2} \theta_{12} e^{2 i \varphi_{12}}\right) \\
& F=\left(\mathbf{I}_{-}^{1} \mathbf{I}_{-}^{2}\right) \times \frac{3}{2}\left(\sin ^{2} \theta_{12} e^{-2 i \varphi_{12}}\right)
\end{aligned}
$$




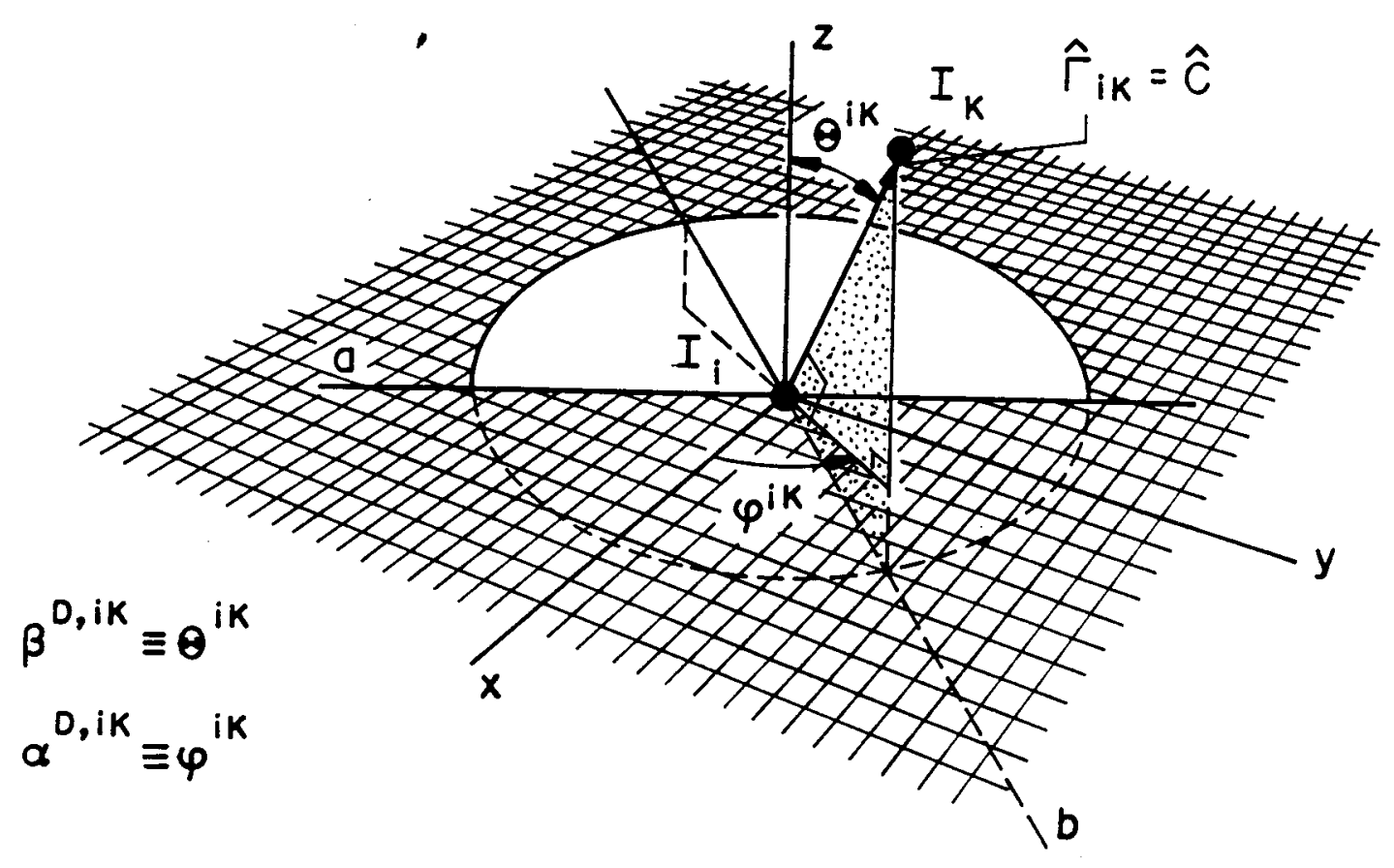

Figura 1.2: Relação entre o sistema do laboratório (LAB) e o sistema de eixos principais $(S E P)$.

Observe que a presença da interaçāo dipolar atua como um efeito pertubativo no hamiltoniano do sistema, pois $\left(\frac{\gamma_{1} \gamma_{2} \hbar^{2}}{r_{12}^{3}}\right)$ corresponde a interação do momentum nuclear com um campo de aproximadamente 1 gauss $\left(10^{-4} T\right)$, enquanto o Hamiltoniano Zeeman $\mathcal{H}_{Z}=-\gamma_{1} \hbar B_{0} I_{z}^{1}-\gamma_{2} \hbar B_{0} \mathbf{I}_{z}^{2}$ corresponde a uma interaçāo com um campo da ordem de $10^{4}$ gauss $(1 T)$.

No sistema de um monocristal, isto é com um só valor para $r_{12}$ entre dois núcleos de spin $\frac{1}{2}$ colocados no campo estático $\mathbf{B}_{0}$, acoplados via $\mathcal{H}_{D}$, teremos dois casos a serem analisados.

\subsubsection{Sistema de dois núcleos $\operatorname{com}\left(\uparrow_{1} \neq \uparrow_{2}\right)$.}

$\mathrm{Na}$ ausência da interação dipolar temos:

$$
\mathcal{H}_{Z}\left|m_{1} m_{2}\right\rangle=\hbar\left(m_{1} \omega_{1}+m_{2} \omega_{2}\right)\left|m_{1} m_{2}\right\rangle
$$

onde $\omega_{1}>\omega_{2}$ sendo $\omega_{i}=-\gamma_{i} \mathbf{B}_{0}$ e $E_{12}=-\hbar B_{0}\left(\gamma_{1} m_{1}+\gamma_{2} m_{2}\right)$, que está esquematizado ${ }^{4}$ na figura (1.3).

Quando aplicamos um campo de rádio-freqüência $B_{1} \cos \omega t$ paralelo ao eixo $x$ do LAB, obtemos uma série de linhas de ressonância, figura (1.3). A linha cheia na figura (1.3) liga

\footnotetext{
${ }^{4}$ Usaremos a notação $\left|\varepsilon_{1}, \varepsilon_{2}\right\rangle$, tal que $\left|m_{1} m_{2}\right\rangle=\frac{1}{2}\left|\varepsilon_{1}, \varepsilon_{2}\right\rangle$ onde $\left\{\left|\varepsilon_{1}, \varepsilon_{2}\right\rangle\right\} \rightarrow\{|++\rangle,|+-\rangle,|-+\rangle,|--\rangle\}$, que formam o conjunto de bases ortonormais do nosso sistema de dois núcleos de spin $\frac{1}{2}$.
} 


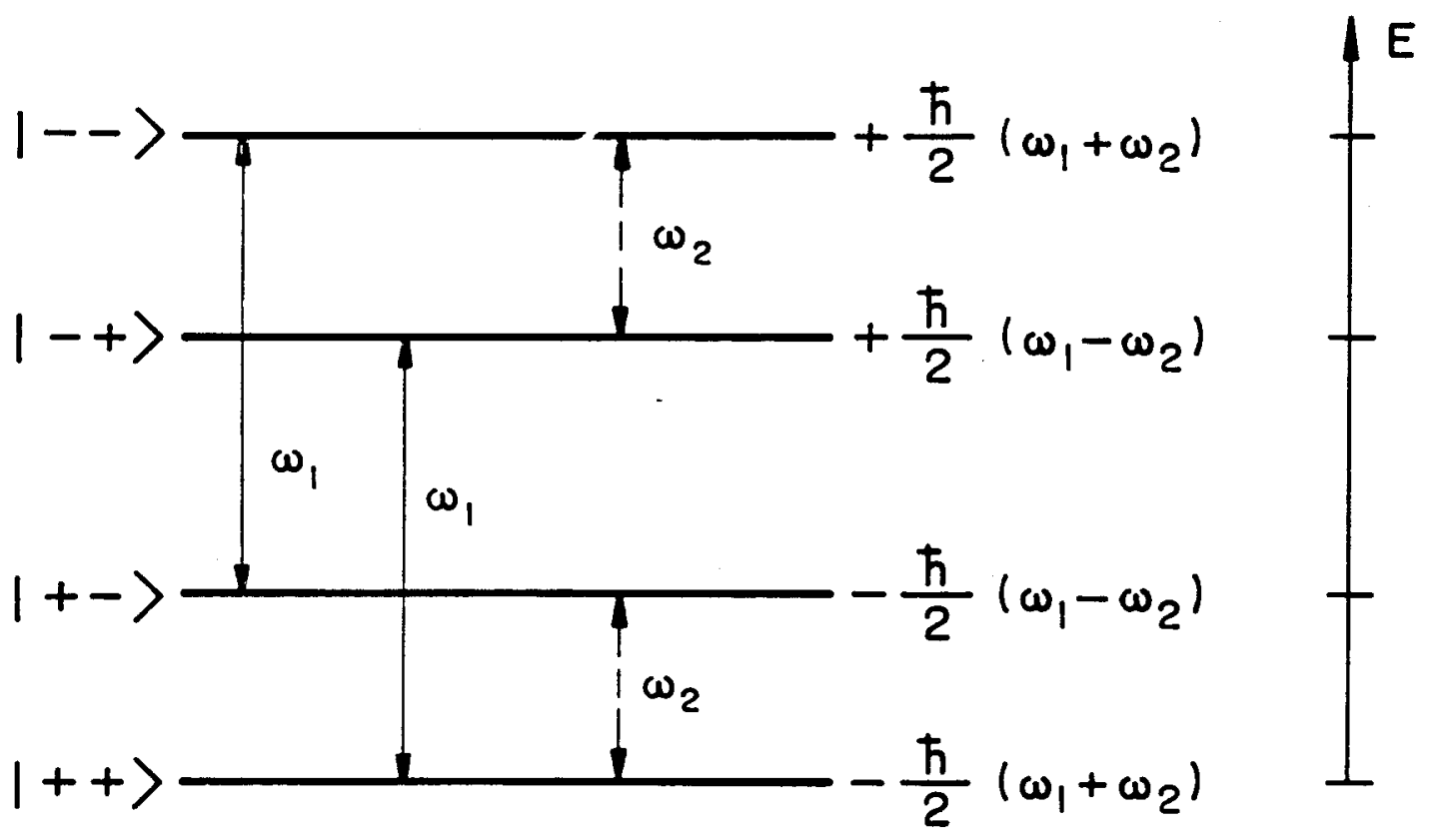

Figura 1.3: Níveis de energia de um sistema de dois núcleos de spin $\frac{1}{2}$, com $\gamma_{1} \neq \gamma_{2}$, num campo magnético externo $\mathbf{B}_{0}=B_{0} \hat{\mathbf{z}}$.

níveis para os quais $\left\langle m_{1} m_{2}\left|I_{1 x}\right| m_{1} m_{2}\right\rangle \neq 0^{5}$ e por sua vez a linha pontilhada liga níveis para os quais $\left\langle m_{1} m_{2}\left|I_{2 x}\right| m_{1} m_{2}\right\rangle \neq 0$. Verifica-se a existência de duas freqüências distintas de Bohr $\omega_{1}$ e $\omega_{2}$, correspondendo às ressonâncias individuais de cada spin nuclear.

Agora, levando-se em conta $\mathcal{H}_{D}$ e o fato dos níveis não serem degenerados, o efeito da interação dipolar é obtido em primeira ordem calculando-se os elementos diagonais de $\mathcal{H}_{D},\left\langle m_{1} m_{2}\left|\mathcal{H}_{D}\right| m_{1} m_{2}\right\rangle$, que de (1.42) fica claro que só o termo $\mathbf{A}$ tem uma contribuição diferente de zero para os elementos da diagonal, resultando:

$$
\left\langle m_{1} m_{2}\left|\mathcal{H}_{D}\right| m_{1} m_{2}\right\rangle=\Omega \hbar m_{1} m_{2}
$$

onde $\Omega=\frac{\hbar \mu_{0}}{16 \pi} \frac{\gamma_{1} \gamma_{2}}{r_{12}^{3}}\left(1-3 \cos ^{2} \theta_{12}\right)$ e por sua vez sendo $\mathcal{H}_{D} \ll \mathcal{H}_{z}$, temos $\Omega \ll \omega_{1}-\omega_{2}$, que resulta nos seguintes deslocamentos de níveis:

$$
\begin{aligned}
& \left\langle--\left|\mathcal{H}_{D}\right|--\right\rangle=+\hbar \Omega, \\
& \left\langle-+\left|\mathcal{H}_{D}\right|-+\right\rangle=-\hbar \Omega, \\
& \left\langle+-\left|\mathcal{H}_{D}\right|+-\right\rangle=-\hbar \Omega, \\
& \left\langle++\left|\mathcal{H}_{D}\right|++\right\rangle=+\hbar \Omega .
\end{aligned}
$$

$\mathrm{Na}$ figura (1.4), linhas cheias representam transições correspondentes a:

$$
\left\langle m_{1} m_{2}\left|I_{1 x}\right| m_{1} m_{2}\right\rangle \neq 0
$$

\footnotetext{
${ }^{5}$ A Probabilidade de transição entre os níveis m e n é dada por $P_{m n} \propto \gamma_{1} \gamma_{2} B_{1}^{2}\left(\left\langle\psi_{m}\left|I_{1 x}\right| \psi_{n}\right\rangle\right)^{2}$.
} 
com freqüências $\omega_{1}+2 \Omega$ e $\omega_{1}-2 \Omega$. Para as linhas pontilhadas temos transições correspondentes a:

$$
\left\langle m_{1} m_{2}\left|I_{2 x}\right| m_{1} m_{2}\right\rangle \neq 0
$$

com freqüências $\omega_{2}+2 \Omega$ e $\omega_{2}-2 \Omega$. Resulta que o espectro de ressonância é composto por dois "doublets" centrados em $\omega_{1}$ e $\omega_{2}$, espaçados de $4 \Omega$; conforme figura (1.5).

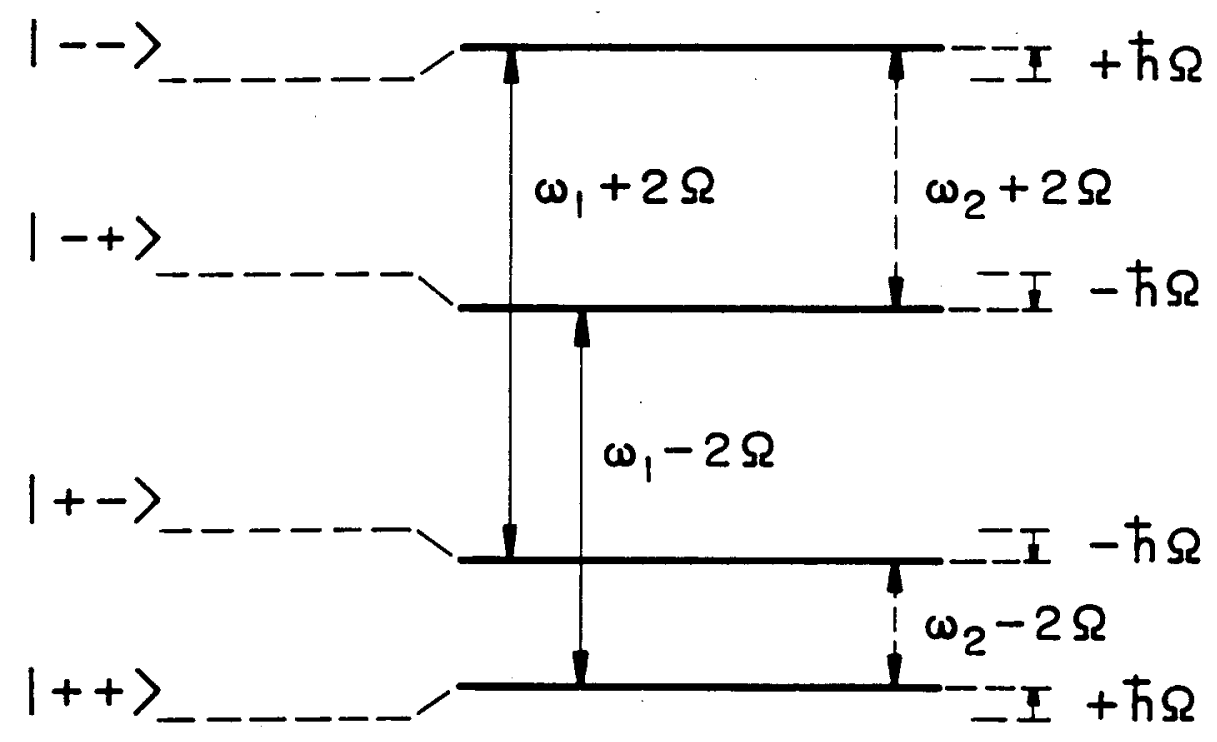

Figura 1.4: Niveis de energia de um sistema de dois núcleos de spin $\frac{1}{2}$, num campo magnético externo $\mathbf{B}_{0}$ e com $\gamma_{1} \neq \gamma_{2}$ levando-se em conta o termo de primeira ordem da interação dipolar entre os spins nucleares. Para as linhas cheias temos transiçóes correspondentes $\grave{a}\left\langle m_{1} m_{2}\left|I_{1 x}\right| m_{1} m_{2}\right\rangle \neq 0$ e para linhas pontilhadas transições correspondentes $\grave{a}$ $\left\langle m_{1} m_{2}\left|I_{2 x}\right| m_{1} m_{2}\right\rangle \neq 0$.

Temos assim que a interação dipolo-dipolo resulta numa estrutura fina no espectro de ressonância magnética.

\subsubsection{Sistema de dois núcleos com $\eta_{1}=\gamma_{2}$.}

$\mathrm{Na}$ ausência da interação dipolar temos

$$
\mathcal{H}_{Z}\left|m_{1} m_{2}\right\rangle=\hbar \omega\left(m_{1}+m_{2}\right)\left|m_{1} m_{2}\right\rangle
$$

onde $\omega_{1}=\omega_{2}=\omega=-\gamma \mathbf{B}_{0}$ e $E_{12}=-\hbar \gamma B_{0}\left(m_{1}+m_{2}\right)$.

Na figura (1.6) vemos quatro transiçōes, que correspondem a uma frequêencia angular $\omega$, que resulta por sua vez no espectro de uma única linha, como representado na figura $(1.7-\mathbf{a})$. 

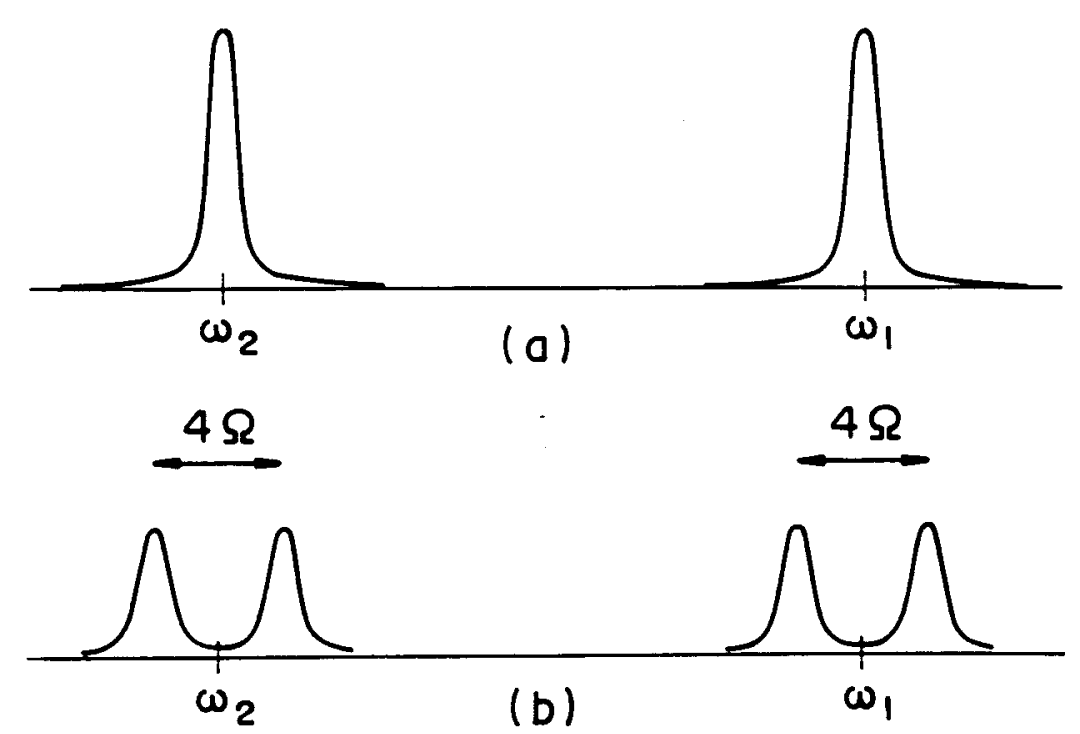

Figura 1.5: Posições das linhas de ressonância que são observadas para um sistema de dois spins nucleares com $\gamma_{1} \neq \gamma_{2}$, para os casos: (a) na ausência de interação dipolar $e$ (b) com a presença de interação dipolar (transições correspondentes as setas da figura (1.4)).

Quando introduzimos a interação dipolar criamos modificações que podem ser analisadas a partir da (1.50), com resultados dados pela tabela (1.1) e figura (1.8):

\begin{tabular}{|l|c|c|c|c|}
\hline $\mathcal{H}_{D}$ & $|++\rangle$ & $|-+\rangle$ & $|+-\rangle$ & $|\overline{\boldsymbol{T}}-\rangle$ \\
\hline$|++\rangle$ & $\hbar \Omega$ & 0 & 0 & 0 \\
\hline$|-+\rangle$ & 0 & $-\hbar \Omega$ & $-\hbar \Omega$ & 0 \\
\hline+-$\rangle$ & 0 & $-\hbar \Omega$ & $-\hbar \Omega$ & 0 \\
\hline$|--\rangle$ & 0 & 0 & 0 & $\hbar \Omega$ \\
\hline
\end{tabular}

Tabela 1.1: Valores de $\left\langle m_{1} m_{2}\left|\mathcal{H}_{D}\right| m_{1} m_{2}\right\rangle=\Omega \hbar m_{1} m_{2}$.

Observe que nesse caso nāo estamos somente considerando o termo $\mathrm{A}$, pois nesse caso temos um sistema degenerado precisamos então considerar também o termo $B$, resultando nos termos fora da diagonal $\left\langle+-\left|\mathcal{H}_{D}\right|-+\right\rangle$ e $\left\langle-+\left|\mathcal{H}_{D}\right|+-\right\rangle$, pois:

$$
\begin{aligned}
& \langle+-|B|-+\rangle=-\hbar \Omega\langle+-\mid+-\rangle, \\
& \langle-+|B|+-\rangle=-\hbar \Omega\langle-+\mid-+\rangle,
\end{aligned}
$$

isso mostra a degenerescência dos níveis $|+-\rangle$ e $|-+\rangle$ do nosso sistema nas bases:

$$
\left\{\left|\varepsilon_{1}, \varepsilon_{2}\right\rangle\right\}=\{|++\rangle,|-+\rangle,|+-\rangle,|--\rangle\} \text {. }
$$




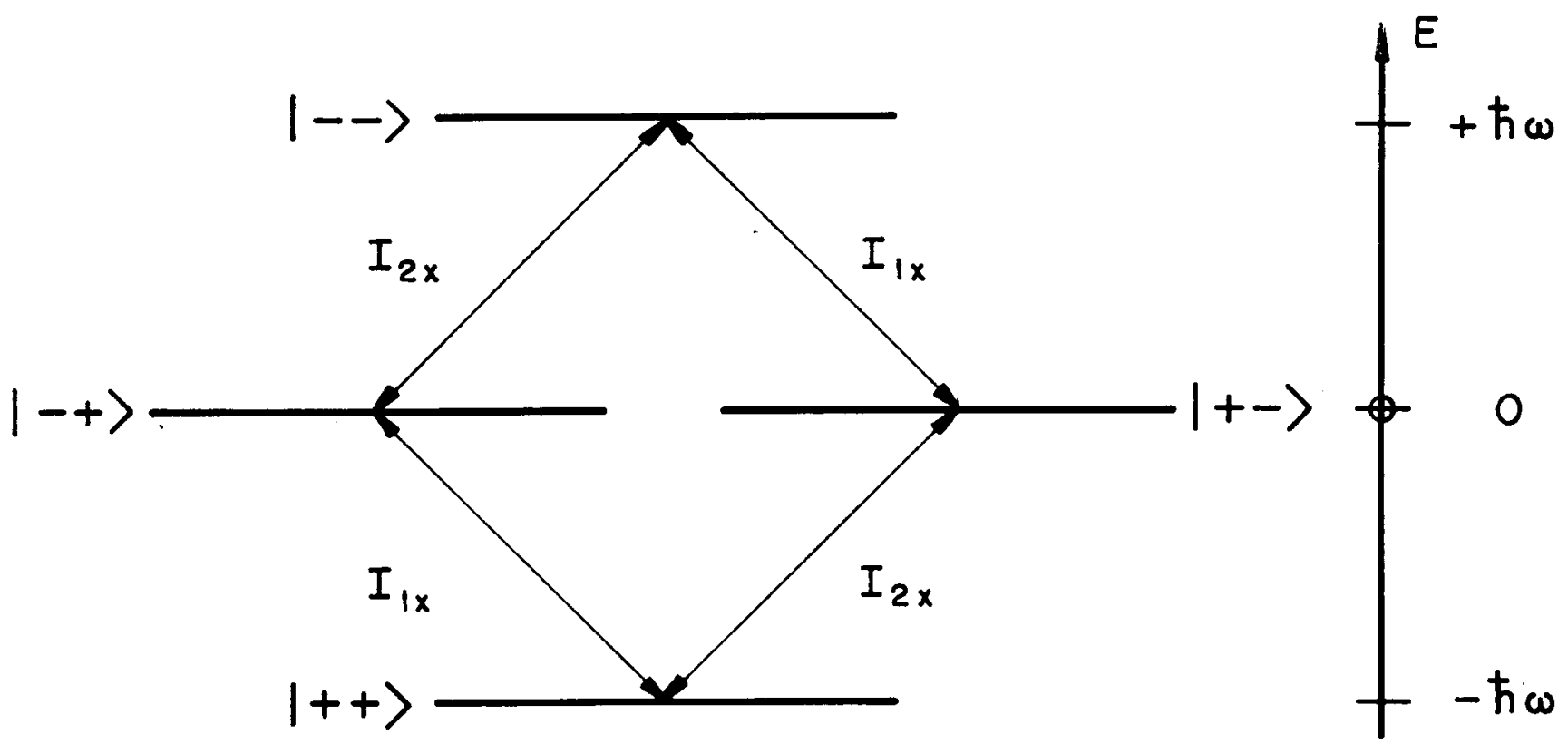

Figura 1.6: Sistema de dois núcleos de spin $\frac{1}{2} \operatorname{com}\left(\gamma_{1}=\gamma_{2}\right)$, na ausência de interação dipolar, resultando numa mesma freqüência ângular de Bohr $\omega_{0}=-\gamma B_{0}$ e num nível duplamente degeneracio.

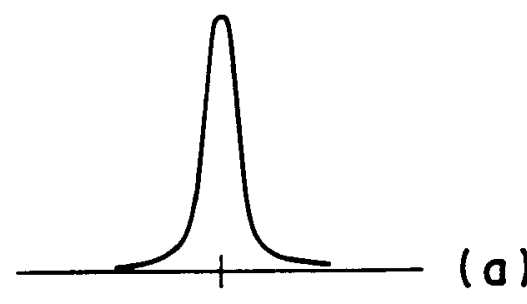

(1)

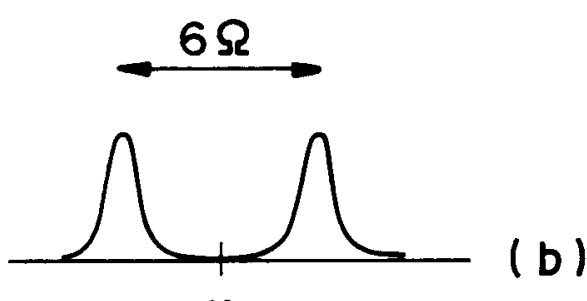

$\omega$

Figura 1.7: Forma do espectro de RMN que é observado para um sistema de dois núcleos de spin $\frac{1}{2}$, que possuem $\gamma_{1}=\gamma_{2}$, colocado num campo magnético estático $\mathbf{B}_{0}$. Na ausencia de interação dipolar observamos um espectro de uma única linha (a). Na presença da interação dipolar (b) a linha se divide com separação de $6 \Omega$. 
Para analisarmos os efeitos criados pela introdução de $\mathcal{H}_{D}$, precisamos quebrar essa degenerescência. Para tal criamos bases a partir da combinação linear das bases antigas $|-+\rangle \mathrm{e}|+-\rangle$; em outras palavras, limitamos-nos ao sub-espaço $\{|+-\rangle,|-+\rangle\}$.

Lembrando-se que $\mathcal{H}_{D}, \mathrm{~S}_{z}$ e $\mathrm{S}^{2}$ comutam:

$$
\left[\mathcal{H}_{D}, \mathrm{~S}^{2}\right]=\left[\mathcal{H}_{D}, \mathrm{~S}_{z}\right]=0
$$

podemos construir a nova base a partir dos auto-estados comuns a $\mathbf{S}^{2}$ e $\mathbf{S}_{z}$, onde $\mathbf{S}=$ $S_{1}+S_{2}$ é o spin nuclear total do sistema e $S^{2}=S_{1}^{2}+S_{2}^{2}+2 S_{1} \cdot S_{2}$, onde:

$$
\mathbf{S}^{2}=\hbar_{2}\left(\begin{array}{cccc}
2 & 0 & 0 & 0 \\
0 & 1 & 1 & 0 \\
0 & 1 & 1 & 0 \\
0 & 0 & 0 & 2
\end{array}\right)
$$

Observe que assim como $\mathcal{H}_{D}, \mathrm{~S}^{2}$ também possui uma degenerescência nos estados $|+-\rangle$ e $|-+\rangle$ que deve ser quebrada. Para tal diagonalizamos essa sub-matriz comum a $S^{2}$ e $\mathcal{H}_{D}$, definindo-se nova base $|S M\rangle$, tal que satisfaça:

$$
\begin{aligned}
& \mathbf{S}_{1}^{2}|S M\rangle=\mathbf{S}_{2}^{2}|S M\rangle=\frac{3}{4} \hbar|S M\rangle, \\
& \mathbf{S}^{2}|S M\rangle=S(S+1) \hbar^{2}|S M\rangle, \\
& \mathbf{S}_{z}|S M\rangle=M \hbar|S M\rangle,
\end{aligned}
$$

onde $-S \leq M \leq+S$.

Obtemos assim para os auto-vetores:

$$
\begin{aligned}
& \left|\psi_{1}\right\rangle=\frac{1}{\sqrt{2}}[|+-\rangle+|-+\rangle], \\
& \left|\psi_{2}\right\rangle=\frac{1}{\sqrt{2}}[|+-\rangle-|-+\rangle],
\end{aligned}
$$

que de (1.63), (1.64) e (1.65) resulta:

\begin{tabular}{|c|c|c|c|c|}
\hline \multirow{2}{*}{ auto-vetor } & \multicolumn{3}{|c|}{ auto-valores } & $|S M\rangle$ \\
\cline { 2 - 5 } & $S(S+1) \hbar^{2}$ & $\delta E$ & $M \hbar$ & \\
\hline$\left|\psi_{1}\right\rangle=\frac{1}{\sqrt{2}}[|+-\rangle+|-+\rangle]$ & $2 \hbar^{2}$ & $-2 \hbar \Omega$ & 0 & $|10\rangle$ \\
$\left|\psi_{2}\right\rangle=\frac{1}{\sqrt{2}}[|+-\rangle-|-+\rangle]$ & 0 & 0 & 0 & $|00\rangle$ \\
\hline
\end{tabular}

Observamos aqui a degenerescência dupla associada a $M=0$, para $S_{z}$. Para o caso de $S=1$, existem ainda as posssibilidades de $M= \pm 1$, que são associados às duas 
sub-matrizes unidimensionais restantes:

$$
\begin{aligned}
\left\langle++\left|\mathcal{H}_{D}\right|++\right\rangle & =2 \hbar^{2}, \\
\left\langle--\left|\mathcal{H}_{D}\right|--\right\rangle & =2 \hbar^{2}, \\
\left\langle++\left|S^{2}\right|++\right\rangle & =\hbar \Omega \\
\left\langle--\left|S^{2}\right|--\right\rangle & =\hbar \Omega
\end{aligned}
$$

que possuem os mesmos auto-valores e auto-vetores tanto em $\left|\varepsilon_{1}, \varepsilon_{2}\right\rangle$ com $|S, M\rangle$. Resulta assim a nova base ortonormal $|S, M\rangle$, comum a $S^{2}, S_{z}$ e $\mathcal{H}_{D}$, dadas por:

$$
\begin{aligned}
|00\rangle & =\frac{1}{\sqrt{2}}[|+-\rangle-|-+\rangle], \\
|10\rangle & =\frac{1}{\sqrt{2}}[|+-\rangle+|-+\rangle], \\
|11\rangle & =|++\rangle, \\
|1-1\rangle & =|--\rangle,
\end{aligned}
$$

onde o conjunto $|1 M\rangle$ constitui os estados tripletos, que são simétricos com respeito à mudança de dois spins e $|00\rangle$ é denominado singleto que é antisimétrico com relação à mudança de dois spins.

Desses novos estados resulta o esquema apresentado na figura (1.8), onde observamos duas transiçōes possíveis correspondente às freqüências de $(\omega+3 \Omega)$ e $(\omega-3 \Omega)$, que resulta num espectro de RMN com um dubleto centrado em $\omega$ com separação de $6 \omega$, conforme figura (1.7-b).

\subsubsection{Significado do Alfabeto Dipolar}

Considerando-se o caso anterior onde $\gamma_{1}=\gamma_{2}$, questionamos que pares de estado são conectados pelos termos do alfabeto dipolar.

Inicialmente vemos que o termo $A$, que é proporcional a $I_{1 z} I_{2 z}$, é um termo diagonal tornando-se responsável por conexões de estados $\left|m_{1}, m_{2}\right\rangle$ com $\left\langle m_{1}, m_{2}\right|$, conforme é mostrado na figura (1.9). Por sua vez o termo $\mathbf{B}$, que é proporcional a $\mathbf{I}_{1}^{+} \mathbf{I}_{2}^{-}+\mathbf{I}_{1}^{-} \mathbf{I}_{2}^{+}$, conecta estados $\left|m_{1}, m_{2}\right\rangle$ com estados $\left\langle m_{1}+1, m_{2}-1\right|$ ou $\left\langle m_{1}-1, m_{2}+1\right|$, pois:

$$
\begin{aligned}
\left\langle m_{1}, m_{2}|\mathbf{B}| m_{1}, m_{2}\right\rangle= & \frac{1}{4}\left(1-3 \cos ^{2} \theta\right) \\
& \times\left\{\left[I(I+1)-m_{1}\left(m_{1}+1\right)\right]^{\frac{1}{2}}\right. \\
& \times\left[I(I+1)-m_{2}\left(m_{2}-1\right)\right]^{\frac{1}{2}}\left\langle m_{1}, m_{2} \mid m_{1}+1, m_{2}-1\right\rangle \\
& +\left[I(I+1)-m_{1}\left(m_{1}-1\right)\right]^{\frac{1}{2}} \\
& \left.\times\left[I(I+1)-m_{2}\left(m_{2}+1\right)\right]^{\frac{1}{2}}\left\langle m_{1}, m_{2} \mid m_{1}-1, m_{2}+1\right\rangle\right\} .
\end{aligned}
$$




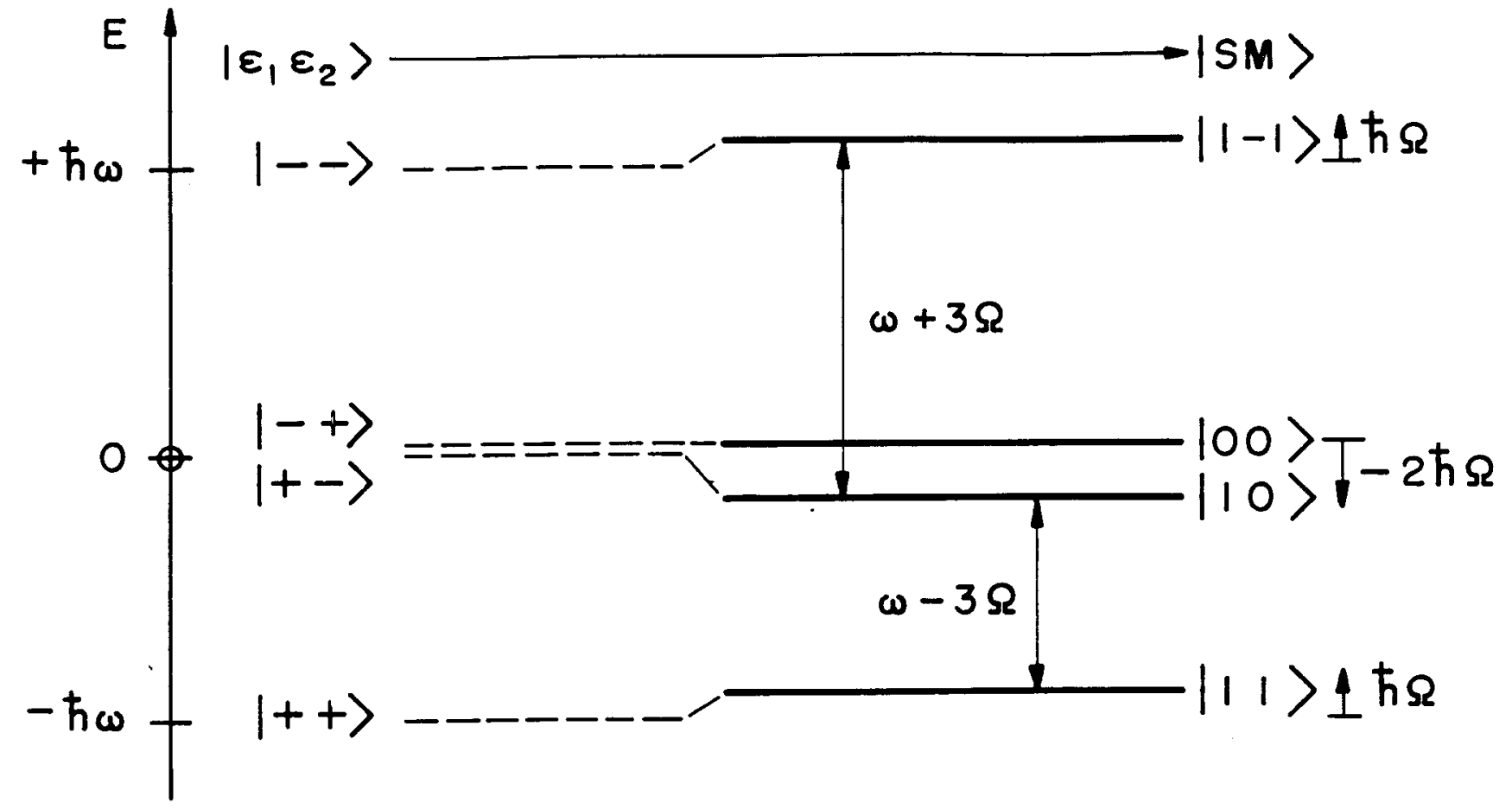

Figura 1.8: Sistema de dois núcleos de spin $\frac{1}{2}$ com $\gamma_{1}=\gamma_{2}$, na presença da interação dipolar. Os níveis se deslocam numa aproximação de primeira ordem conforme indicado no lado direito da figura. Para zero-ordem em $\mathcal{H}_{D}$, os estados estacionários são auto-estados $|S M\rangle$ do spin total. As setas ligam niveis onde $\left\langle S M\left|I_{1 x}+I_{2 x}\right| S M\right\rangle \neq 0$.

Dizemos que o termo $B$ "flipa" os spins nucleares simultaneamente um para cima e outro para baixo, figura (1.9). Observe que esse termo não possui elementos de matriz diagonal nas bases $m_{1}, m_{2}$, mas possui elementos fora da diagonal, entre os estados que são degenerados, contribuindo para a transição entre eles. Vimos também que o termo $\mathbf{B}$ serviu para quebrar a degenerescência dos estados degenerados $|+-\rangle \mathrm{e}|-+\rangle$.

Os termos $\mathbf{C} \& \mathbf{D}$ são proporcionais a $\left(I_{2 z} I_{1}^{+}+I_{2}^{+} I_{1 z}\right)$ e $\left(I_{1 z} I_{2}^{-}+I_{1}^{-} I_{2 z}\right)$, sendo $\tilde{\mathbf{D}}=\mathbf{C}$, conectando estados $\left|m_{1}, m_{2}\right\rangle$ com estados $\left|m_{1}, m_{2}-1\right\rangle+\left|m_{1}+1, m_{2}\right\rangle$, pois :

$$
\begin{aligned}
\left\langle m_{1}, m_{2}|\mathbf{C}| m_{1}, m_{2}\right\rangle= & -\frac{3}{2 \sqrt{2}}\left(\sin 2 \theta_{12} e^{i \varphi}\right) \\
& \times\left\{m _ { 2 } \left[\left(I(I+1)-m_{1}\left(m_{1}+1\right)\right]^{\frac{1}{2}}\left\langle m_{1}, m_{2} \mid m_{1}+1, m_{2}\right\rangle\right.\right. \\
& \left.+m_{1}\left[I(I+1)-m_{2}\left(m_{2}+1\right)\right]^{\frac{1}{2}}\left\langle m_{1}, m_{2} \mid m_{1}, m_{2}+1\right\rangle\right\}, \\
\left\langle m_{1}, m_{2}|\mathbf{D}| m_{1}, m_{2}\right\rangle= & +\frac{3}{2 \sqrt{2}}\left(\sin 2 \theta_{12} e^{-i \varphi}\right) \\
& \times\left\{m _ { 2 } \left[\left(I(I+1)-m_{1}\left(m_{1}-1\right)\right]^{\frac{1}{2}}\left\langle m_{1}, m_{2} \mid m_{1}-1, m_{2}\right\rangle\right.\right. \\
& \left.+m_{1}\left[I(I+1)-m_{2}\left(m_{2}-1\right)\right]^{\frac{1}{2}}\left\langle m_{1}, m_{2} \mid m_{1}, m_{2}-1\right\rangle\right\} .
\end{aligned}
$$

Isto é, os termos C e D "flipam" somente um spin conectando estados que diferem de energia $\hbar \omega$, figura (1.10).

Os termos $E \& \mathbf{F}$ sāo proporcionais a $I_{1}^{+} I_{2}^{+}$e $I_{1}^{-} I_{2}^{-}$, sendo $\bar{E}=F$, conectando estados $\left|m_{1}, m_{2}\right\rangle$ a estados $\left|m_{1} \pm 1, m_{2} \pm 1\right\rangle$, pois: 


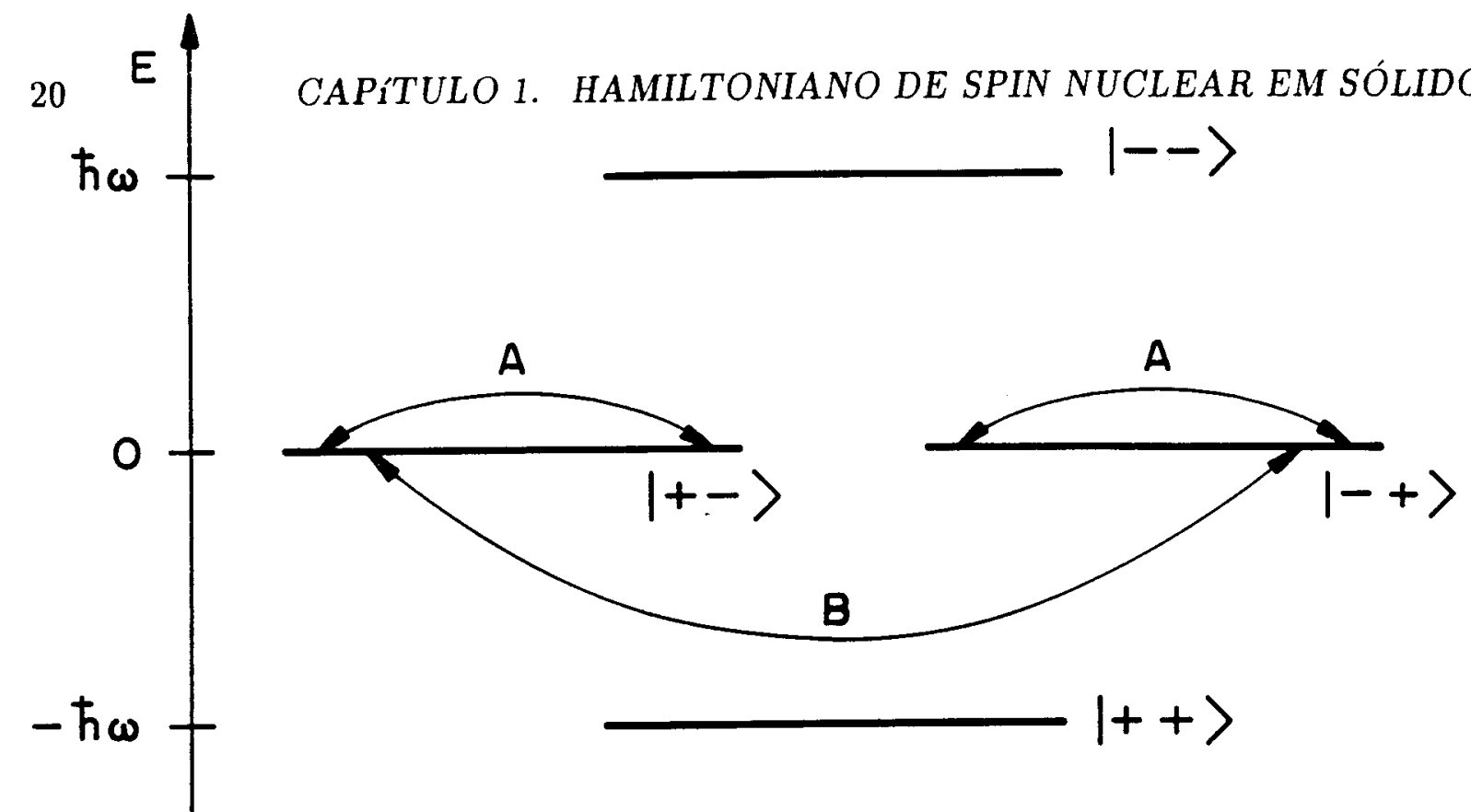

Figura 1.9: Transiçóes entre estados proporcionadas pelo campo de rádio-freqüência via termos A\&B da interação dipolar, para um sistema de dois núcleos de $\operatorname{spin} \frac{1}{2} \operatorname{com} \gamma_{1}=\gamma_{2}$.

$$
\begin{aligned}
\left\langle m_{1}, m_{2}|\mathbf{E}| m_{1}, m_{2}\right\rangle= & \frac{3}{2}\left(\sin ^{2} \theta_{12} e^{2 i \varphi}\right) \\
& \times\left[I(I+1)-m_{1}\left(m_{1}+1\right)\right]^{\frac{1}{2}} \\
& \times\left[I(I+1)-m_{2}\left(m_{2}+1\right)\right]^{\frac{1}{2}}\left(m_{1}, m_{2}\left|m_{1}+1, m_{2}+1\right\rangle,(1.78)\right. \\
\left\langle m_{1}, m_{2}|\mathbf{F}| m_{1}, m_{2}\right\rangle= & \frac{3}{2}\left(\sin ^{2} \theta_{12} e^{2 i \varphi}\right) \\
& \times\left[\left(I(I+1)-m_{1}\left(m_{1}-1\right)\right]^{\frac{1}{2}}\right. \\
& \times\left[I(I+1)-m_{2}\left(m_{2}-1\right)\right]^{\frac{1}{2}}\left\langle m_{1}, m_{2} \mid m_{1}-1, m_{2}-1\right\rangle .
\end{aligned}
$$

Isto é, os termos E\&F "flipam" ambos os spins, conectando estados que diferem na energia de $2 \hbar \omega_{o}$, figura (1.10).

Desses resultados observa-se que os termos do Hamiltoniano Dipolar são termos que provocam mistura de estados através de transiçōes induzidas pela rádio-freqüência. Os termos $\mathbf{C , D}, \mathbf{E}$ e $\mathbf{F}$, em particular, são termos fora da diagonal permitindo misturas de estados exatos $|++\rangle,|--\rangle$ e estados de primeira ordem $|10\rangle$ e $|00\rangle$.

Para calcularmos a mistura de estados, obtemos inicialmente a função de onda de ordem zero corretas, isto é consideramos para a pertubação $\mathcal{H}_{D}$ o sistema já com os níveis degenerados abertos em $|00\rangle$ e $|10\rangle$, conforme (1.72) e (1.73), resultando:

$$
\psi_{n} \approx \varphi_{n}^{(0)}+\sum_{m \neq n} \frac{\left\langle\varphi_{m}^{(0)}\left|\mathcal{H}_{D}\right| \varphi_{n}^{(0)}\right\rangle}{E_{n}^{0}-E_{m}^{(0)}} \varphi_{m}^{(0)},
$$




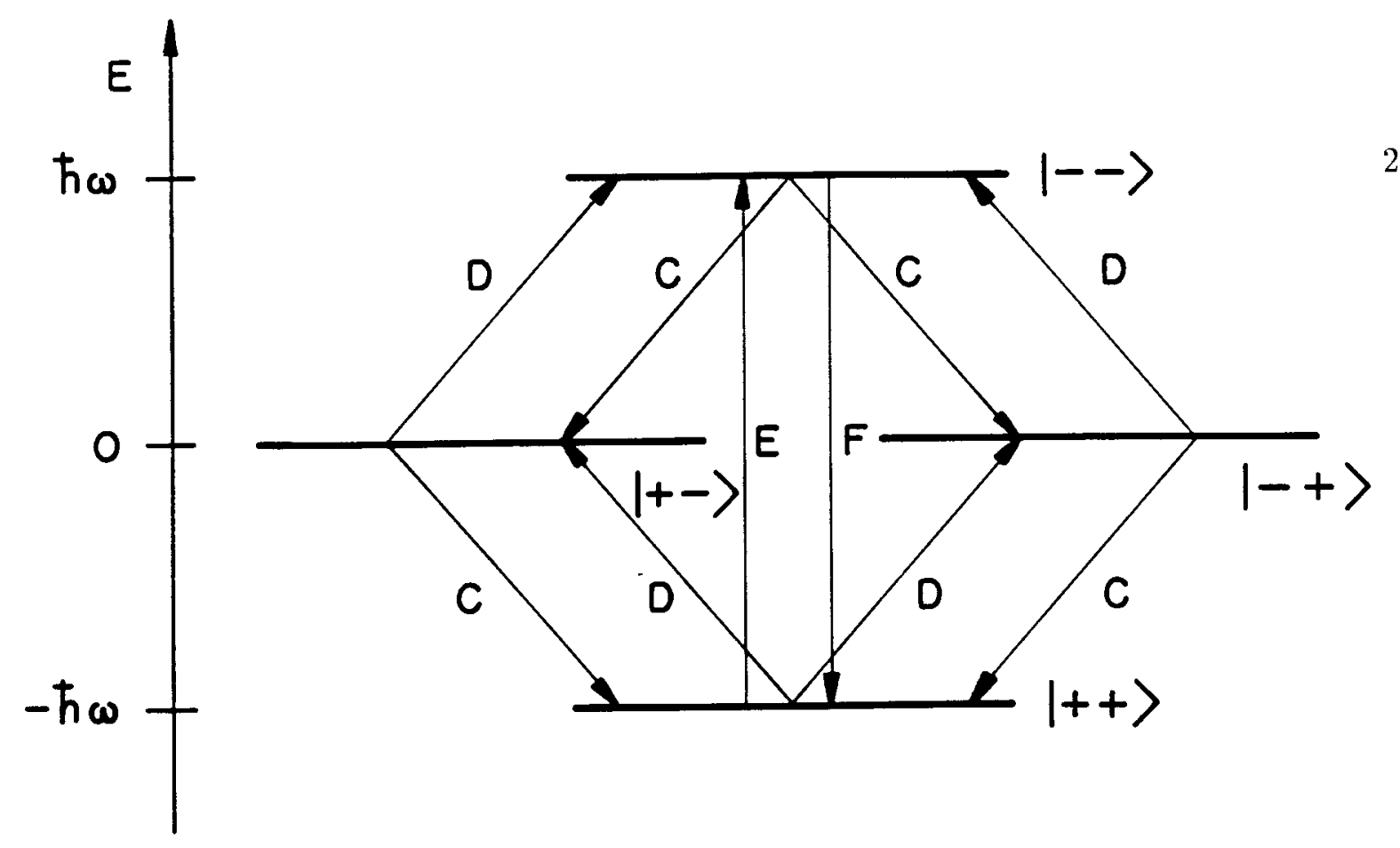

Figura 1.10: Transições entre estados proporcionadas pelo campo de rádio-freqüência via termos $\mathbf{C}, \mathbf{D}, \mathbf{E}$ e $\mathbf{F}$ da interação dipolar, para um sistema de dois núcleos de spin $\frac{1}{2}$ com $\gamma_{1}=\gamma_{2}$.

onde:

$$
\begin{array}{ll}
\varphi_{1}^{(0)}=|11\rangle, & E_{1}^{(0)}=\hbar\left(\omega_{0}+\Omega\right), \\
\varphi_{2}^{(0)}=|00\rangle, & E_{2}^{(0)}=0, \\
\varphi_{3}^{(0)}=|10\rangle, & E_{3}^{(0)}=-2 \hbar \Omega, \\
\varphi_{4}^{(0)}=|1-1\rangle, & E_{4}^{(0)}=\hbar\left(\Omega+\omega_{0}\right) .
\end{array}
$$

Realizando-se as contas obtemos os seguintes estados misturados:

$$
\begin{aligned}
\left|\psi_{1}\right\rangle & =\alpha_{1}|++\rangle+\alpha_{2}|+-\rangle+\alpha_{3}|-+\rangle+\alpha_{4}|--\rangle, \\
\left|\psi_{2}\right\rangle & =\beta_{1}|++\rangle+\beta_{2}|+-\rangle+\beta_{3}|-+\rangle+\beta_{4}|--\rangle, \\
\left|\psi_{3}\right\rangle & =\gamma_{2}|+-\rangle+\gamma_{3}|-+\rangle \\
\rightarrow\left|\psi_{4}\right\rangle & =\delta_{1}|++\rangle+\delta_{2}|+-\rangle+\delta_{3}|-+\rangle+\delta_{4}|--\rangle .
\end{aligned}
$$


onde:

$$
\begin{array}{ll}
\alpha_{1}=-\frac{\gamma^{2} \hbar E(\theta, \varphi)}{2 r^{3} \omega_{0}} & \alpha_{2}=-\frac{\gamma^{2} \hbar C(\theta, \varphi)}{2 r^{3}\left(\omega_{0}+\Omega\right)} \\
\alpha_{3}=\alpha_{2} & \alpha_{4}=1 \\
\beta_{1}=-\frac{\gamma^{2} \hbar C(\theta, \varphi)}{r^{3}\left(\omega_{0}+3 n\right)} & \beta_{2}=-\frac{1}{2 \sqrt{2} \Omega}\left\{1-\frac{\gamma^{2} \hbar}{r^{3}}\left[\frac{A(\theta)}{2}+B(\theta)\right]\right\} \\
\beta_{3}=-\beta_{2} & \beta_{4}=-\frac{\gamma^{2} \hbar D(\theta, \varphi)}{r^{3}\left(\omega_{0}-3 \Omega\right)} \\
\gamma_{2}=\frac{1}{\sqrt{2}} & \gamma_{3}=-\frac{1}{\sqrt{2}} \\
\delta_{1}=1 & \delta_{2}=-\frac{2 \gamma^{2} \hbar D(\theta, \varphi)}{\sqrt{2} r^{3}\left(\omega_{0}+3 \Omega\right)} \\
\delta_{3}=\delta_{2} & \delta_{4}=-\frac{\gamma^{2} \hbar F(\theta, \varphi)}{2 r^{3} \omega_{0}}
\end{array}
$$

Para analisarmos a regra de seleção das transições entre níveis pensamos num único núcleo, onde a hamiltonina da rádio-freqüência é tada por:

$$
\mathcal{H}_{r f}(t)=2 \gamma B_{1} \cos \omega t \mathrm{I}_{1 x}
$$

onde:

$$
\mathbf{I}_{1 x}=\frac{1}{2}\left(\mathbf{I}_{1}^{+}+\mathrm{I}_{1}^{-}\right)
$$

Resulta assim para a probabilidade de transição $P_{m n}$, do nosso sistema com estados misturados $\left|\psi_{n}\right\rangle$ :

$$
\begin{gathered}
P_{m n} \propto B_{1}^{2}\left[\left\langle\psi_{m}\left|\mathrm{I}_{1 x}\right| \psi_{n}\right\rangle\right]^{2} . \\
P_{12}=P_{23}=P_{14}=P_{24} \propto \gamma^{2} \frac{B_{1}^{2} B_{l o c}^{2}}{B_{0}^{2}} \sim 10^{-14} \\
P_{34}=P_{13} \propto \gamma^{2} B_{1}^{2} \sim 10^{-7} .
\end{gathered}
$$

Observe que a mistura de estados provocada pelos termos C, D, E e F da interação dipolar propicia à rádio-freqüência induzir transiçōes de estados $|++\rangle$ e $|--\rangle$, que antes eram proibidas. De (1.89) vemos porém que essas transições possuem probabilidades muito pequena, são ditas transições fracas, que ocorrem numa freqüência de $-2 \Omega$ e $2 \omega$, figura (1.11). Por sua vez, para as transições fortes (1.90) vimos que ocorrem numa freqüência de $\omega \pm 3 \Omega$. O efeito desses termos $C, D, E$ e $F$ é então permitir absorções perto de $-2 \Omega$ e $2 \omega$, conforme figura (1.12), que são desprezíveis para a nossa análise. 


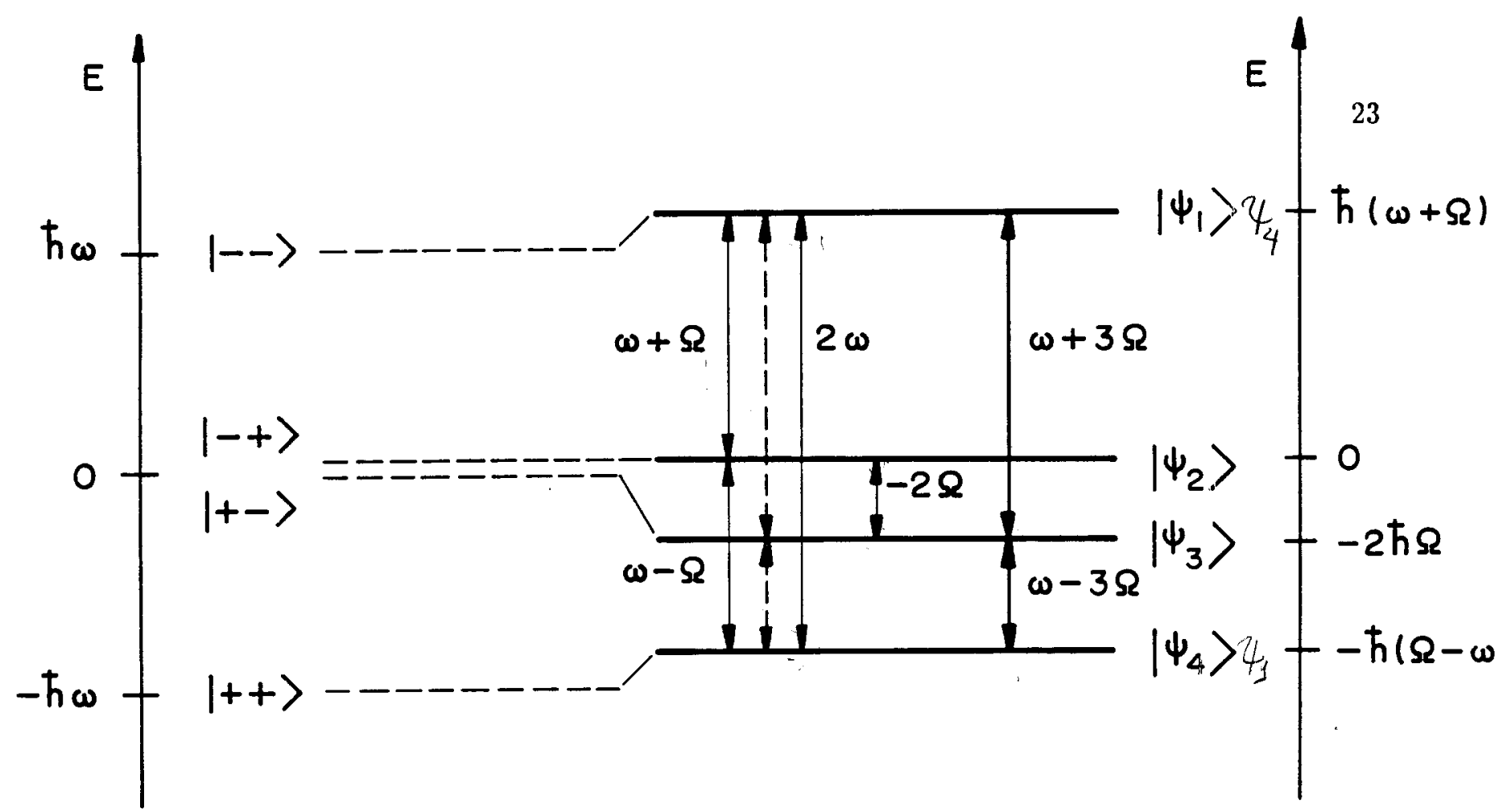

Figura 1.11: Transições de um núcleo do sistema de dois núcleos com $\gamma_{1}=\gamma_{2}=\gamma$. A transição indicada pela linha cheia e linha dupla são transições propiciadas pela mistura de estados devidos à interação dipolar. A linha pontilhada é a transição devida à rádio-freqüência sem levar em conta a mistura de estados.

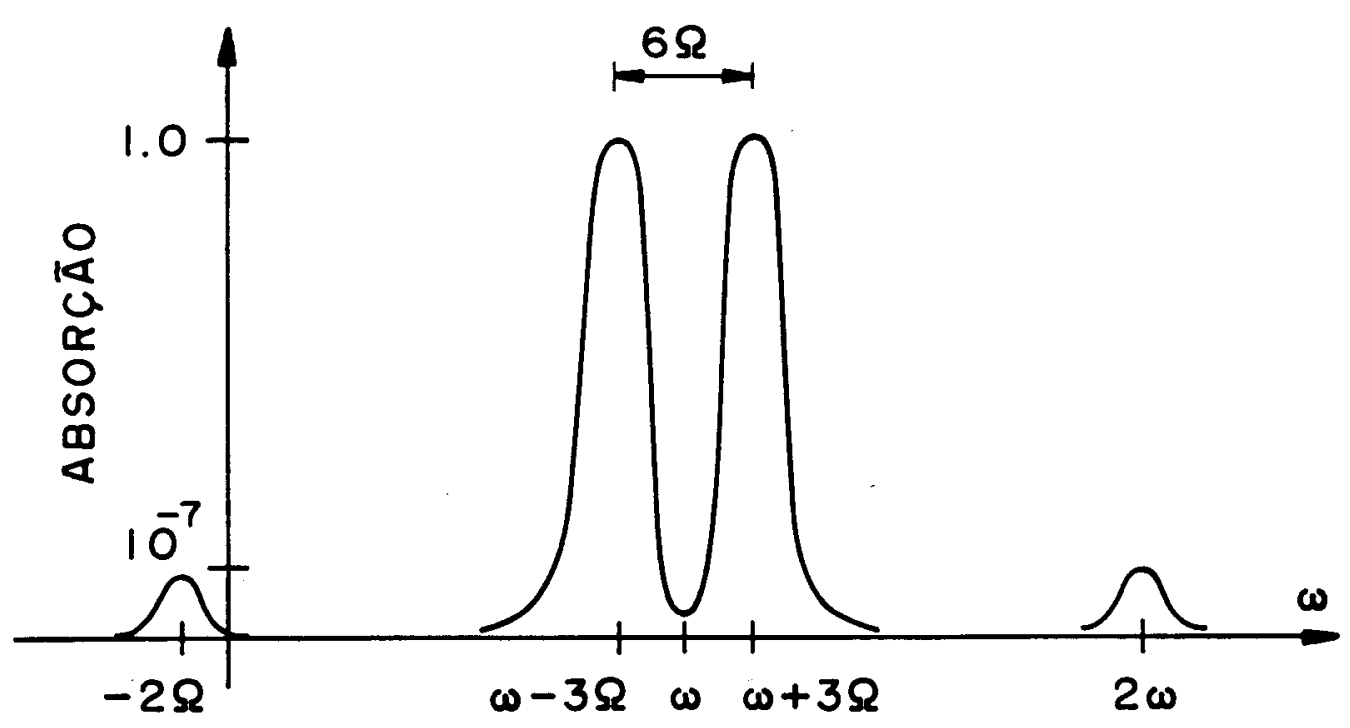

Figura 1.12: Absorção versus freqüência incluindo a interação dipolar. 


\subsection{Deslocamento Químico em Sólidos}

\subsubsection{Introdução}

Estaremos agora interessados em discutir a interação anisotrópica de proteção magnética nuclear, que é responsável pelos deslocamentos químicos. A principal razão para ocorrência dos deslocamentos químicos em RMN reside nos elétrons.

Num experimento de RMN, a freqüência de um spin nuclear não é somente determinada pela constante giromagnética $\gamma$, mas também pela interação do spin nuclear com os elétrons circundantes, que são responsáveis por correntes de elétrons induzidas nas moléculas da amostra pelo campo aplicado ou, por outras palavras, em termos de al teração (polarização) ${ }^{6}$ do comportamento orbital dos elétrons produzidos pelo campo aplicado. Isto é, o campo magnético no sítio do núcleo é alterado e inferior ao campo aplicado ${ }^{7}$, resultando:

$$
\mathbf{B}=(1-\sigma) \mathbf{B}_{0},
$$

onde $\sigma$, dito tensor de proteção magnética, adimensional, simétrico e de segunda ordem, representa os deslocamentos anisotrópicos da freqüência de ressonância de um núcleo numa molécula com relação ao núcleo nú.

Esse deslocamento químico é representado pelo hamiltoniano:

$$
\mathcal{H}_{n Q}=\hbar \gamma \mathbf{I} \cdot \sigma \cdot \mathbf{B}_{0} .
$$

Em líquidos de baixa viscosidade, a rotação isotrópica anula a parte anisotrópica do tensor de deslocamento químico, resultando para a parte isotrópica:

$$
\sigma=\frac{1}{3} t r \sigma=\frac{1}{3}\left(\sigma_{a a}+\sigma_{b b}+\sigma_{c c}\right)
$$

onde $\sigma_{a a}, \sigma_{b b}$ e $\sigma_{c c}$ são os elementos principais do tensor de deslocamento químico. Porém, em sólidos, usualmente não existe rotação molecular isotrópica suficiente para anular a parte anisotropica deslocamento químico. Assim a freqüência de ressonância de um certo spin num sólido depende da direção do campo externo com relação à amostra. Isto nada mais é que uma manisfestação experimental de que a nuvem de elétrons em volta de um núcleo geralmente não possui simetria esférica. Portanto, o tensor de deslocarnento químico nos fornece informações sobre a simetria local da nuvem de elétrons em torno do núcleo e este, por sua vez, apresenta uma descrição muito mais detalhada das ligaçōes químicas de certos átomos, do que o deslocamento químico obtido em solução.

\footnotetext{
${ }^{8}$ Sendo extremamente complexas as circulações dos elétrons induzidas por um campo magnético numa molécula, recorre-se freqüentemente à aproximaçāo de considerar o efeito total como o resultado da polarização do movimento orbital dos elétrons associados formalmente aos vários átomos e ligações químicas.

${ }^{7}$ Tal fenômeno é dito diamagnetismo, isso só é possível por estarmos tratando de material diamagnético (moléculas que possuem elétrons com spins compensados).
} 
Apesar de estarmos interessados em anular essas interações anisotrópicas, elas possuem informações sobre a geometria, estrutura e movimento no sólido, que refletem em outro aspecto de espectroscopia revelando informações sobre a própria interação. Convém aqui lembrar que nosso interesse são espectros isotrópicos de alta resolução permitindo a obtenção de informações sobre a estrutura química da molécula, isto é prevalece o deslocamento químico isotrópico.

\subsubsection{Interação de Deslocamento Químico}

Como sempre, definimos a direção do campo magnético externo $\mathbf{B}_{0}$, sobre a direção do eixo $z$ do referencial do laboratório (LAB). Nesse sistema de referência o tensor deslocamento químico é representado por:

$$
\sigma=\left(\begin{array}{lll}
\sigma_{x x} & \sigma_{x y} & \sigma_{x z} \\
\sigma_{y x} & \sigma_{y y} & \sigma_{y z} \\
\sigma_{z x} & \sigma_{z y} & \sigma_{z z}
\end{array}\right),
$$

que resulta para o $\mathcal{H}_{D Q}$ na condição de $\mathbf{B}_{0}=B_{0} \hat{\mathbf{z}}$ :

$$
\mathcal{H}_{D Q}=\hbar \gamma B_{0}\left(I_{z} \sigma_{z z}+I_{x} \sigma_{x z}+I_{y} \sigma_{y z}\right)
$$

Em geral $\sigma$ não é necessariamente simétrico, porém suas componentes antisimétricas contribuem em segunda ordem para o deslocamento da freqüência de ressonância, podendo ser ignoradas mesmo no caso de $\sigma$ ser antisimétrico. Assim, $\sigma_{z z}$ é que rege o comportamento do deslocamento da freqüência de ressonância que depende da orientação de $\mathbf{B}_{0}$ com relação aos eixos principais do tensor $\sigma$.

Uma maneira de verificar essa dependência geométrica é escrever $\sigma_{z z}$ em função das componentes principais $\sigma_{i i}$. Sendo o elemento de tensor;

$$
\sigma_{z z}(L A B)=\sum_{i=1}^{3} a_{i j} a_{i z} \sigma_{i i}(S E P)
$$

onde $a_{i z}$ e $a_{k z}$ são cossenos diretores dado por:

$$
a_{i z}=\hat{\mathbf{e}}_{i} \cdot \hat{\mathbf{z}}=\cos \beta_{i z}=\cos \beta_{i}
$$

conforme figura(1.13), resulta para $\sigma_{z z}$ :

$$
\begin{aligned}
\sigma_{z z}(L A B) & =(\hat{\mathbf{a}} \cdot \hat{\mathbf{z}})^{2} \sigma_{a a}+(\hat{\mathbf{b}} \cdot \hat{\mathbf{z}})^{2} \sigma_{b b}+(\hat{\mathbf{c}} \cdot \hat{\mathbf{z}})^{2} \sigma_{c c} \\
& =\sin ^{2} \beta \cos ^{2} \gamma \sigma_{a a}+\sin ^{2} \beta \sin ^{2} \gamma \sigma_{b b}+\cos ^{2} \beta \sigma_{c c} .
\end{aligned}
$$

Para um sistema de estrutura com simetria axial, temos $\sigma_{a a}=\sigma_{b b}=\sigma_{\perp}$ e $\sigma_{c c}=\sigma_{\|}$, resulta para (1.98):

$$
\sigma_{z z}(L A B)=\sigma+\frac{1}{2} \delta\left[3 \cos ^{2} \beta-1+\eta \sin ^{2} \beta \cos 2 \gamma\right]
$$



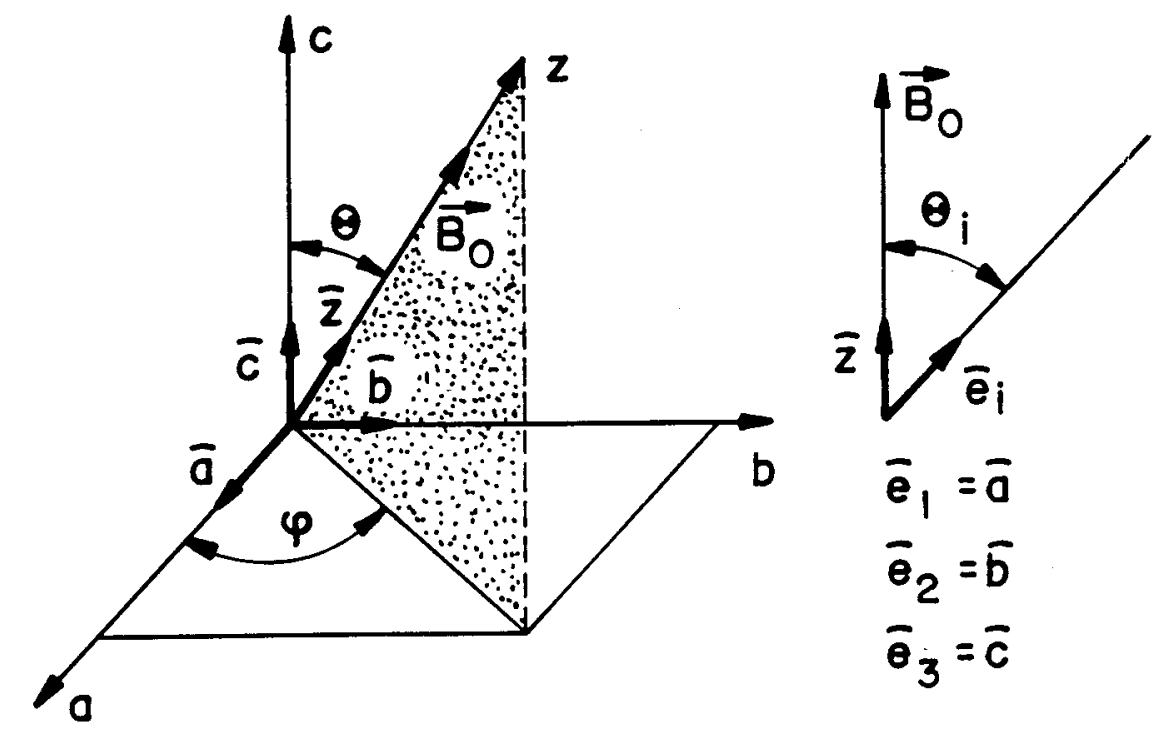

Figura 1.13: Cossenos diretores, onde: $(\hat{\mathbf{a}} \cdot \hat{\mathbf{z}})=\sin \beta \cos \gamma,(\hat{\mathbf{b}} \cdot \hat{\mathbf{z}})=\sin \beta \sin \gamma$, $(\hat{\mathbf{a}} \cdot \hat{\mathbf{z}})=\cos \gamma$.

Tratando-se agora do tensor de deslocamento químico no sistema de eixos principais (SEP), vimos no ínicio desse capítulo que o tensor $\mathbf{R}=\sigma$ de segunda ordem é descrito pela componente isotrópica $\sigma$ mais dois parâmetros $\delta$ e $\eta$, onde:

$$
\begin{aligned}
\delta & =\sigma_{c c}-\sigma \\
\eta & =\frac{\sigma_{a c}-\sigma_{b b}}{\delta}
\end{aligned}
$$

onde os elementos diagonais são ordenados de maneira que $\left|\sigma_{c c}-\sigma\right| \geq\left|\sigma_{a a}-\sigma\right| \geq\left|\sigma_{b b}-\sigma\right|$.

Observe que $\delta$ é responsável pela anisotropia do deslocamento químico e $\eta$ pela assimetria, da condição de estrutura com simetria axial, resulta de (1.100) e (1.101), que $\delta=\frac{2}{3}\left(\sigma_{\|}-\sigma_{\perp}\right)$.

De $(1.10),(1.11)$ e (1.12) vimos que as componentes esféricas são expressas em termos de três parâmetros:

$$
\begin{aligned}
\sigma_{00}(S E P) & =\sigma, \\
\sigma_{20}(S E P) & =\sqrt{\frac{3}{2}} \eta, \\
\sigma_{2 \pm 1}(S E P) & =\frac{1}{2} \eta \delta .
\end{aligned}
$$

Na notação de tensores irredutíveis, o Hamiltoniano de Deslocamento Químico de um dado núcleo $(n)$ assume a forma ${ }^{8}$ :

\footnotetext{
${ }^{8}$ Vimóos no apêndice B, equaçōes (B.19)-(B.24) que somente $\ell=0,2$ resultam em $R_{\ell, m}^{D Q} \neq 0$, para um R simétrico.
} 


$$
\mathcal{H}_{D Q}=\gamma_{n} \sum_{\ell=0}^{2} \sum_{m=-\ell}^{+\ell}(-1)^{m} \sigma_{\ell,-m}^{D Q} T_{\ell, m}^{D Q} .
$$

onde $T_{\ell, m}^{D Q}=\mathrm{IB}$ em coordenadas esféricas são dadas de (B.38) por $^{9}$ :

$$
\begin{array}{ll}
T_{00}^{D Q}=\frac{1}{\sqrt{3}}\left(T_{x x}+T_{y y}+T_{z z}\right) & =\frac{1}{\sqrt{3}} I_{0} B_{0} \\
T_{02}^{D Q}=\frac{1}{\sqrt{6}}\left(2 T_{z z}-T_{x x}-T_{y y}\right) & =\sqrt{\frac{2}{3}} I_{0} B_{0} \\
T_{2 \pm 1}^{D Q}=\mp \sqrt{\frac{1}{2}}\left(T_{x z} \pm i T_{y z}\right) & =\frac{1}{\sqrt{2}} I_{ \pm 1} B_{0} \\
T_{2 \pm 2}^{D Q}=\frac{1}{2}\left(T_{x x}-T_{y y} \pm i\left(T_{x y}+T_{y x}\right)\right. & =0 \\
T_{1 \pm 1}^{D Q}=\frac{1}{2}\left(T_{z x}-T_{x z} \pm i\left(T_{z y}-T_{y z}\right)\right. & =0 \\
T_{10}^{D Q}=\frac{i}{\sqrt{2}}\left(T_{x y}-T_{y x}\right) & =0 .
\end{array}
$$

Transformando $\sigma_{\ell m}(S E P)$ para o sistema do laboratório (LAB), temos:

$$
\sigma_{\ell m}(L A B)=\sum_{m^{\prime}} \mathcal{D}_{m^{\prime},-m}^{\ell} \sigma_{\ell m^{\prime}}(S E P)
$$

resultando para $(1.105)$ :

$$
\begin{array}{rlrl}
\mathcal{H}_{D Q} & =\gamma_{n} \sum_{\ell=0,2} \sum_{m=-\ell}^{+\ell}(-1)^{m} T_{\ell, m} \sum_{m^{\prime}} \mathcal{D}_{m^{\prime},-m}^{\ell} \sigma_{\ell, m} & \\
& =\gamma_{n} T_{00} \sigma_{00}+\gamma_{n} \sum_{m=-2}^{+2}(-1)^{m} T_{2 m} \sum_{m^{\prime}} \mathcal{D}_{m^{\prime},-m}^{2} \sigma_{2 m^{\prime}} & \underline{\ell} & \underline{m} \\
& =\omega_{0} \sigma I_{0} & 0 & 0 \\
& +\omega_{0}\left\{\sqrt{\frac{2}{3}} I_{0} \delta\left[\sqrt{\frac{3}{2}} \mathcal{D}_{00}^{2}+\frac{\eta}{2}\left(\mathcal{D}_{20}^{2}+\mathcal{D}_{-20}^{2}\right)\right]\right. & 2 & 0 \\
& -\frac{1}{\sqrt{2}} I_{-1} \delta\left[\sqrt{\frac{3}{2}} \mathcal{D}_{01}^{2}+\frac{\eta}{2}\left(\mathcal{D}_{21}^{2}+\mathcal{D}_{-21}^{2}\right)\right] & 2 & -1 \\
& \left.-\frac{1}{\sqrt{2}} I_{+1} \delta\left[\sqrt{\frac{3}{2}} \mathcal{D}_{0-1}^{2}+\frac{\eta}{2}\left(\mathcal{D}_{2,-1}^{2}+\mathcal{D}_{-2,-1}^{2}\right)\right]\right\} . & 2 & 1
\end{array}
$$

Não existem os termos $\ell=2, m= \pm 2$, porque vimos que $T_{2, \pm 2}^{D Q}=0$. Considerando-se somente as componentes do campo induzido pelos elétrons $\left(\sigma \cdot \mathbf{B}_{0}\right)$ no sítio do núcleo, que sāo paralelas ou anti-paralelas ao campo aplicado $\mathbf{B}_{0}=B_{o} \hat{\mathbf{z}}$, restringimos o Hamiltoniano aos termos seculares, $\mathcal{H}_{D Q \text {, sec }}$. Utilizando-se dos elementos da matriz de rotação de

\footnotetext{
${ }^{\theta}$ Definimos o campo magnético externo $\mathbf{B}_{0}$ paralelo ao eixo $\mathbf{z}, \mathbf{B}_{0}=B_{0} \hat{\mathbf{z}}$, e também $\mathbf{I}_{z}=\mathbf{I}_{0}$.
} 
Wigner, $\mathcal{D}_{\ell, m}^{(2)}$ (Apêndice A), resulta:

$$
\begin{aligned}
\mathcal{H}_{D Q, \text { sec }} & =\omega_{0} I_{0}\left\{\sigma+\sqrt{\frac{2}{3}} \sigma_{20}(L A B)\right\}, \\
\sigma_{20}(L A B) & =\sqrt{\frac{3}{8}} \delta\left[\left(3 \cos ^{2} \beta-1\right)+\eta \sin ^{2} \beta \cos 2 \gamma\right] .
\end{aligned}
$$

Comparando-se (1.109), (1.110) com (1.99) vemos que:

$$
\mathcal{H}_{D Q, \text { sec }}=\omega_{0} I_{0} \sigma_{z z}
$$

onde $\sigma_{z z}$ é a componente de "shielding" no sistema de laboratório. Observe que $\mathcal{H}_{D Q \text {,sec }}$ não depende do ângulo de Euler $\alpha$. Isto reflete o fato de que experimentos de RMN são invariantes quando sofrem rotações em torno do campo externo aplicado $\mathbf{B}_{\mathbf{0}}$, figura (A.1).

Considerando-se agora um sistema de dois níveis, $|+\rangle$ e $|-\rangle$, com $\sigma_{z z}>0$, levando-se em conta o termo Zeeman resulta para o Hamiltoniano:

$$
\mathcal{H}=-\omega_{0} \hbar\left(1-\sigma_{z z}\right) \mathbf{I}_{z},
$$

com deslocamentos dos níveis de energia, figura (1.14) dado por:

$$
\begin{aligned}
& \langle+|\mathcal{H}|+\rangle=-\frac{\omega_{0} \hbar}{2}\left(1-\sigma_{z z}\right), \\
& \langle-|\mathcal{H}|-\rangle=+\frac{\omega_{0} \hbar}{2}\left(1-\sigma_{z z}\right)
\end{aligned}
$$

A posição de uma linha no espectro para um núcleo $i$ é governada por $\mathcal{H}_{D Q, s e c}^{i}$, conforme (1.109). Porém precisamos de um referencial para obtermos a posição de linha de $\omega^{i}$ no espectro. Para tal utilizamos-nos de uma $\omega^{\text {ref }}$, que é a posição de linha de uma amostra de referência para o qual $\sigma_{20}^{i}$ é nulo ${ }^{10}$. Assim obtemos de (1.109):

$$
\omega^{i}-\omega^{\text {ref }}=-\omega_{0}\left\{\sigma^{i}+\sqrt{\frac{2}{3}} \sigma_{20}^{i}(L A B)-\sigma_{\text {ref }}\right\}
$$

sendo que $\omega^{i}-\omega^{\text {ref }}$ é o que medimos numa experiência de RMN, pois o deslocamento escalar que medimos não é $\sigma_{z z}^{i}$, mas $\operatorname{sim} \sigma_{z z}^{i}-\sigma_{r e f}^{i}$, conforme figura (1.15). Agora para um núcleo $i$ numa amostra com $\sigma$ axialmente simétrico e com $\sigma_{\text {ref }}=0$, resulta para (1.115):

$$
\frac{\omega^{r e f}-\omega^{i}}{\omega_{0}}=\sigma_{\|}^{i} \cos ^{2} \beta^{i}+\sigma_{\perp}^{i} \sin ^{2} \beta^{i}
$$

donde variando-se $\beta^{i}$, revelamos as posições das linhas $\sigma_{\|}$e $\sigma_{\perp}$, conforme figura (1.16).

\footnotetext{
${ }^{10}$ Como faremos isto, será visto no capítulo 2 .
} 


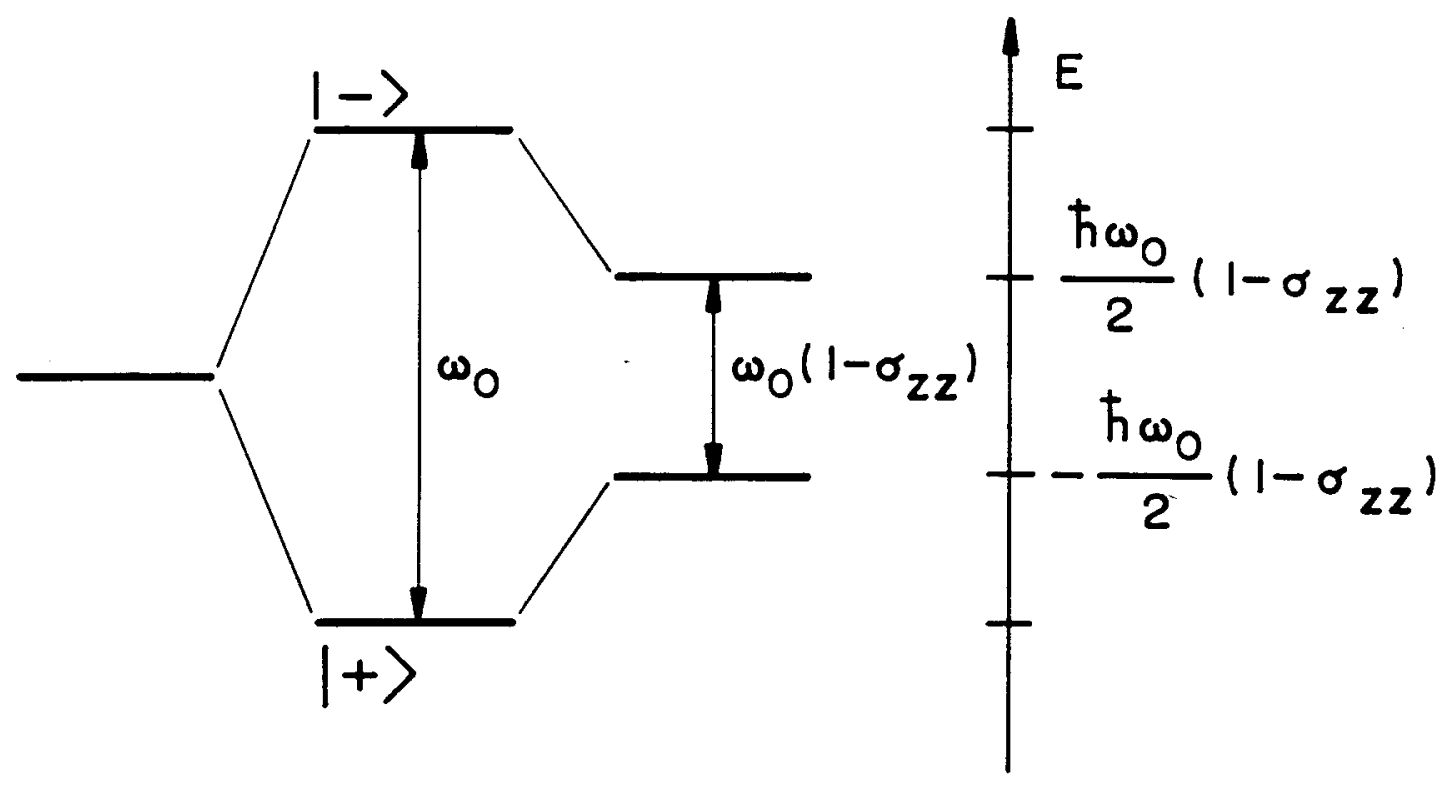

Figura 1.14: Niveis de energia para um sistema de dois níveis sujeitos a interação Zeeman e deslocamento químico.

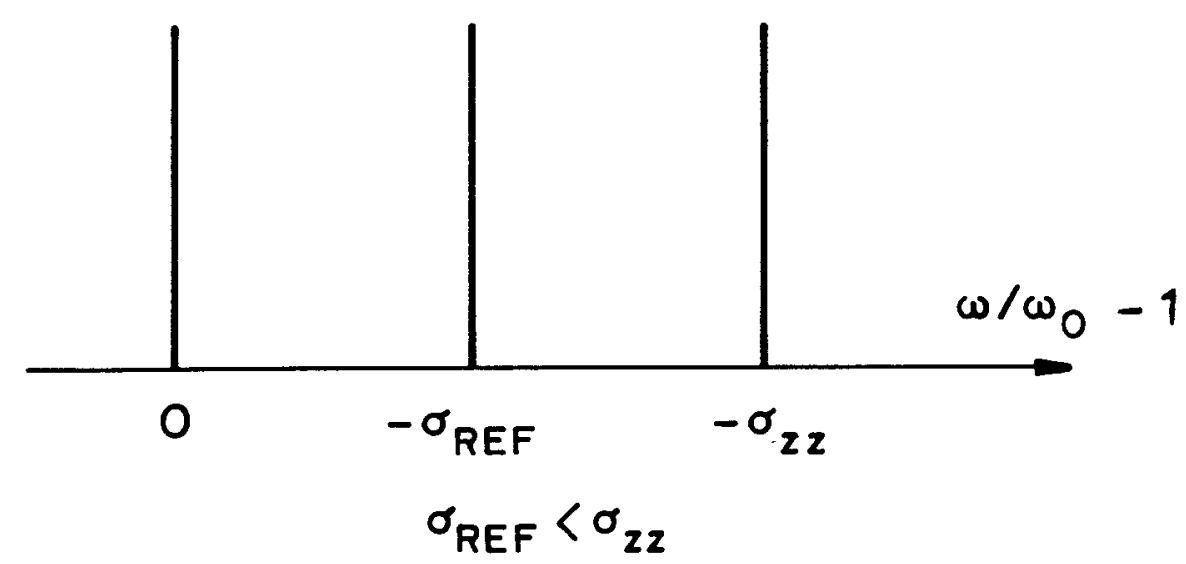

Figura 1.15: Posições das linhas para o núcleo " $n u$ ", $\omega_{0}$, para o núcleo de referência, $\omega_{0}\left(1-\sigma_{r e f}\right)$ e para um núcleo desconhecido, $\omega_{0}\left(1-\sigma_{z z}\right)$. 


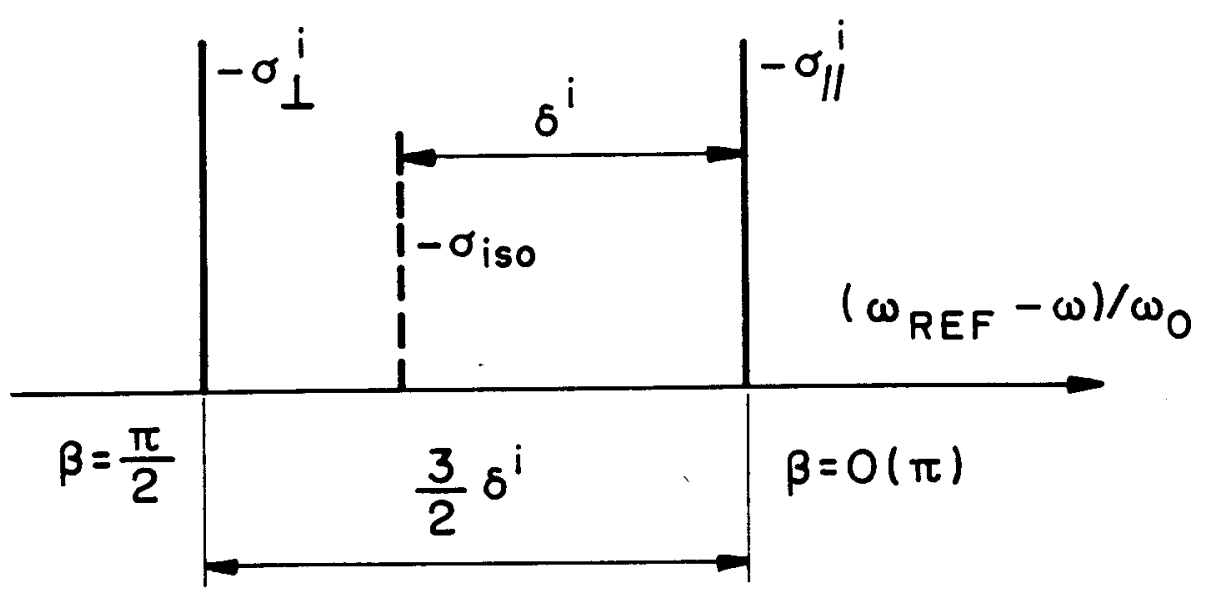

Figura 1.16: Posições das linhas $\sigma_{\perp}$ e $\sigma_{\|}$, onde $\sigma_{\|}>\sigma_{\perp}$ e $\sigma_{i s o} \ll \sigma_{\|} \& \sigma_{\perp}$.

Obtemos assim os parâmetros de "shildeing" das posiçōes espectrais dessa linha, que depende do parâmetro externo $\beta^{i}$, que é a orientação da amostra com relação a $\mathbf{B}_{0}$. Num caso geral (não axialmente simétrico), temos que $\sigma_{20}^{i}(L A B)$ é expresso em termos de $\delta^{i}, \eta^{i}, \beta^{i}, \gamma^{i}$. Embora $\beta^{i}$ e $\gamma^{i}$ são parâmetros que $\omega^{i}-\omega^{\text {ref }}$ depende diretamente, esses não são os parâmetros que realmente estamos interessados.

Os parâmetros que nos interessa realmente são $\sigma^{i}-\delta_{\text {ref }}, \delta^{i}, \eta_{i}$ e os ângulos de Euler $\alpha_{i}^{\prime}, \beta_{i}^{\prime}, \gamma_{i}^{\prime}\left(\equiv \Omega^{\prime}\right)$, que relaciona o sistema da amostra (AMO) arbitrariamente escolhido com o sistema de eixos principais (SEP) do tensor $\sigma^{i}$.

Nessas condiçôes precisamos expressar $\sigma_{20}^{i}$ em termos de $\delta^{i}, \eta^{i}, \Omega_{i}^{\prime}$ e de $\alpha ", \beta ", \gamma "$, que relaciona o sistema de eixos da amostra com o sistema do laboratório, figura (1.17).

Para facilitar nossa vizualização utilizamo-nos dos ângulos polares $\theta$ e $\varphi$ que especificam a direção de um campo magnético externo no referencial da amostra, sendo:

$$
\begin{aligned}
& \beta^{\prime \prime}=\theta, \\
& \gamma^{\prime \prime}=\varphi .
\end{aligned}
$$

Para tal utilizamos-no da matriz de rotação de Wigner $\mathcal{D}_{m^{\prime} 0}^{2}\left(\Omega^{\prime \prime}\right)$, onde $\Omega$ " são os ângulos de Euler que levam o sistema da amostra (AMO) a coincidir com o sistema do laboratório (LAB), resultando:

$$
\sigma_{20}^{i}(L A B)=\sum_{m^{\prime}} \mathcal{D}_{m^{\prime} 0}^{(2)}\left(\Omega^{\prime \prime}\right) \sigma_{2 m^{\prime}}^{i}(A M O)
$$

agora do sistema da amostra (AMO) para o sistema de eixos principais (SEP), utilizandose de (1.102), (1.103) e (1.104):

$$
\sigma_{20}^{i}(L A B)=\sum_{m^{\prime}} \mathcal{D}_{m^{\prime} 0}^{(2)}\left(\Omega^{\prime \prime}\right) \sum_{m^{\prime \prime}} \mathcal{D}_{m^{\prime \prime} m^{\prime}}^{(2)}\left(\Omega_{i}^{\prime}\right) \sigma_{2 m^{\prime \prime}}^{i}(S E P)
$$



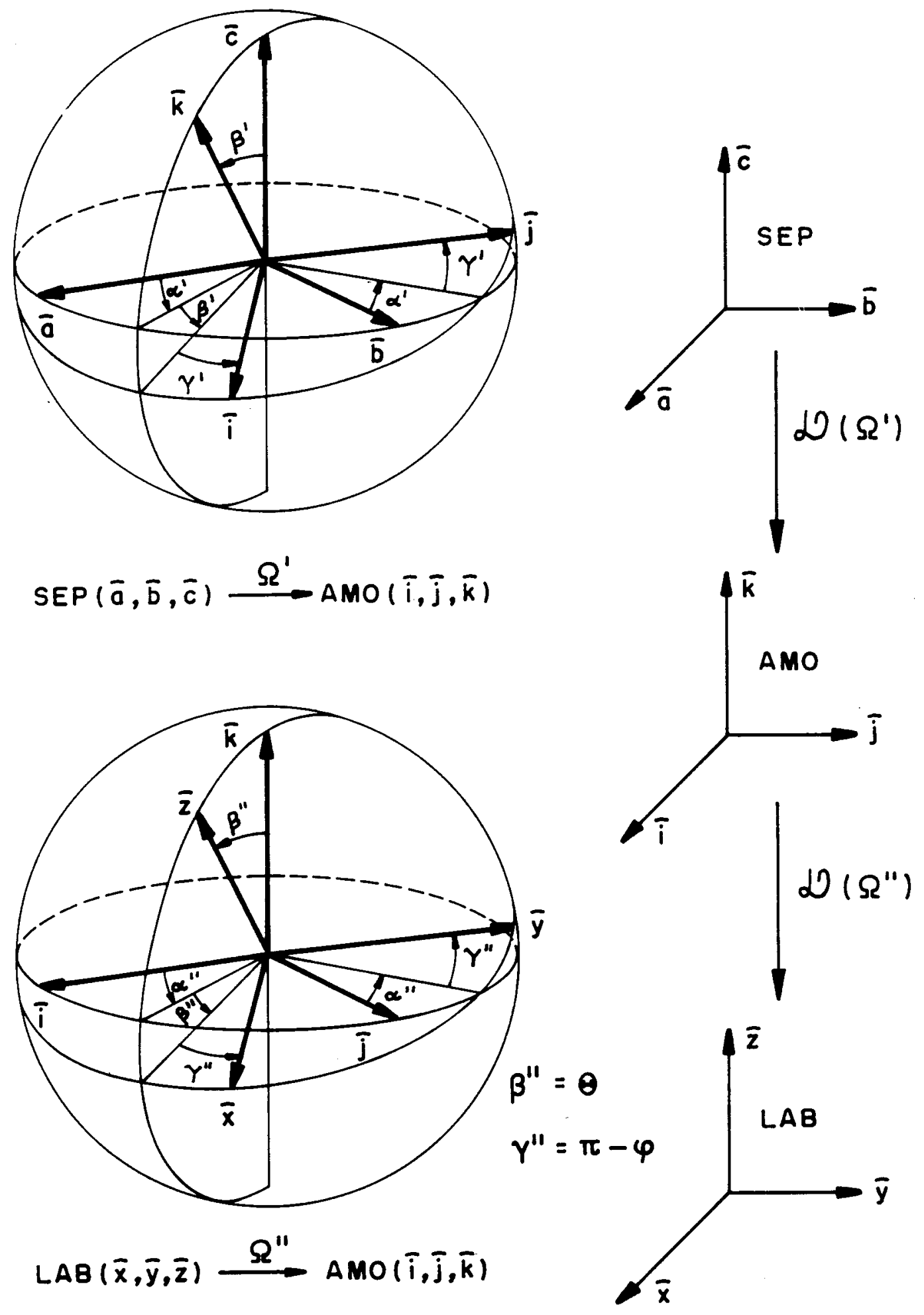

Figura 1.17: Relação dos sistema de eixos principais [(SEP) $(\hat{a}, \hat{b}, \hat{c})]$ e sistema da amostra [(AMO) $(\hat{\imath}, \hat{\jmath}, \hat{k})]$ com orientação relativa ao $(S E P)$ dada pelos ângulos de Euler $\alpha^{\prime}, \beta^{\prime}, \gamma^{\prime}\left(\equiv \Omega^{\prime}\right)$ e por sua vez o sistema do laboratório $\left.[(L A B)](\hat{x}, \hat{y}, \hat{z})\right]$ com orientação de $\hat{z}$ relativa $a(\hat{\imath}, \hat{\jmath}, \hat{k})$ especificado por dois ângulos de Euler, aqui denominado $\theta=\beta$ " $e$ $\varphi=\pi+\gamma "$, de $\alpha ", \beta ", \gamma "$. 


$$
=\sum_{m^{\prime}} \mathcal{D}_{m^{\prime} 0}^{(2)}\left(\Omega^{\prime \prime}\right) \delta^{i}\left\{\sqrt{\frac{3}{2}} \mathcal{D}_{0 m^{\prime}}^{(2)}\left(\Omega_{i}^{\prime}\right)+\frac{1}{2} \eta^{i}\left[\mathcal{D}_{2 m^{\prime}}^{(2)}\left(\Omega_{i}^{\prime}\right)+\mathcal{D}_{-2 m^{\prime}}^{(2)}\left(\Omega_{i}^{\prime}\right)\right]\right\}
$$

Das matrizes de Wigner, verificamos que $\mathcal{D}_{m^{\prime} 0}^{(2)}(\Omega$ ") não depende de $\alpha$ " mas sim de $\beta$ " e $\gamma^{\prime \prime}$. Isto vem dos harmônicos esféricos, que resulta para $\mathcal{D}_{-m^{\prime}, 0}^{(2)}\left(\Omega^{\prime \prime}\right)^{11}$ :

$$
\begin{aligned}
\mathcal{D}_{m^{\prime} 0}^{(2)}\left(\Omega^{\prime \prime}\right) & =(-1)^{m^{\prime}} \sqrt{\frac{4 \pi}{5}} Y_{2 m^{\prime}}\left(\beta^{\prime \prime}, \gamma^{\prime \prime}\right) \\
& =(-1)^{m^{\prime}} \sqrt{\frac{4 \pi}{5}} Y_{2 m^{\prime}}(\theta, \pi-\varphi) \\
& =\sqrt{\frac{4 \pi}{5}} Y_{2-m^{\prime}}(\theta, \varphi) .
\end{aligned}
$$

Por sua vez, resulta para a posição das linhas em função do parâmetros $\delta^{i}, \eta^{i}, \Omega_{i}^{\prime}$ e $\sigma^{i}-\delta_{\text {ref }}$, de (1.121), (1.103), (1.104) e (1.115) com $\sigma_{\text {ref }}=0$ :

$$
\begin{aligned}
\frac{\omega^{\text {ref }}-\omega^{i}}{\omega_{0}}= & \sigma^{i}-\sigma_{\text {ref }}+\sqrt{\frac{8 \pi}{15}} \delta^{i} \sum_{m^{\prime}} Y_{2,-m^{\prime}}(\theta, \varphi) \\
& \times\left\{\sqrt{\frac{3}{2}} \mathcal{D}_{0 m^{\prime}}^{2}\left(\Omega_{i}^{\prime}\right)+\frac{1}{2} \eta^{i}\left[\mathcal{D}_{2 m^{\prime}}^{(2)}\left(\Omega_{i}^{\prime}\right)+\mathcal{D}_{-2 m^{\prime}}^{(2)}\left(\Omega_{i}^{\prime}\right)\right]\right\}
\end{aligned}
$$

que dá as posiçōes das linhas para o caso geral, dependente dos parâmetros externos $\theta$ e $\varphi$. Sua forma de linha será analisada na próxima seção.

\subsection{Espectros de Pó}

\subsubsection{Formas de Linhas}

Uma amostra em pó consiste de um aglomerado de monocristais, aleatoriamente orientados, resultando em todas orientações possíveis dos eixos principais de $\sigma$ em relação a $\mathbf{B}_{0}$. Fazendo-se o sistema de eixos principais (SEP) do tensor $\sigma$ coincidir com os eixos da amostra, isto é $\Omega_{i}^{\prime}=(0,0,0)$. Desde que $\mathcal{D}_{m^{\prime} m^{\prime \prime}}^{2}(0)=\delta_{m^{\prime \prime} m^{\prime}}$, resulta para $(1.122)$ :

$$
\begin{aligned}
\frac{\omega^{\text {ref }}-\omega^{i}}{\omega_{0}} & =\sigma^{i}-\sigma_{\text {ref }}+\sqrt{\frac{8 \pi}{15}} \delta^{i}\left[\sqrt{\frac{3}{2}} Y_{20}(\theta, \varphi)+\frac{1}{2} \eta^{i}\left(Y_{22}(\theta, \varphi)+Y_{2,-2}(\theta, \varphi)\right]\right. \\
& =\sigma^{i}-\sigma_{\text {ref }}+\delta^{i}\left[\frac{1}{2}\left(3 \cos ^{2} \theta-1\right)+\frac{1}{2} \eta_{i} \sin ^{2} \theta \cos 2 \varphi\right]
\end{aligned}
$$

${ }^{11}$ Utilizamo-nos de $\mathcal{D}_{-m 0}^{\ell}\left(\alpha^{\prime \prime}, \beta^{\prime \prime}, \gamma^{\prime \prime}\right)=(-1)^{m} \sqrt{\frac{4 \pi}{2 \ell+1}} Y_{2 m}\left(\beta^{\prime \prime}, \gamma^{\prime \prime}\right)$ conforme Rose [1], pg 60 
donde é facil verificar que a dependência orientacional da posição de linha e dada pelo termo:

$$
\omega=\omega_{0} \delta\left[\frac{1}{2}\left(3 \cos ^{2} \theta-1\right)+\frac{1}{2} \eta \sin ^{2} \theta \cos 2 \varphi\right] .
$$

O espectro de RMN de uma amostra em pó será a superposição das linhas de RMN para todos os núcleos de todos os grãos da amostra. Por sua vez, as direções de um único eixo de todos os tensores $\sigma_{i}$ podem ser graficado sobre uma esfera de raio unitário, resultando numa distribuição constante sobre essa.

A intensidade $I(\omega)$ do espectro quando integrado num intervalo $\omega_{i n i}$ à $\omega_{\text {fin }}$ é proporcional ao número de núcleos cuja linha de $\mathrm{RMN}$ caem nesse intervalo, que é uma cinta circular de espessura $\left(\omega_{f i n}-\omega_{i n i}\right)$, sendo $\omega_{i n i}$ e $\omega_{f i n}$ constantes sobre essa esfra, conforme (1.123). Integrando-se em $\omega$ nesse intervalo constante, obtemos $I(\omega)$ proporcional ao número de núcleos (número de orientaçōes de $\sigma$ ), cuja linha de RMN caem nesse intervalo de freqüência, isto é:

$$
\int_{\omega_{i n i}}^{\omega_{f i n}} I(\omega) d \omega=N \underbrace{\iint d \Omega}_{\omega_{i n i}-\omega_{f i n}}=N \iint \sin \theta d \theta d \varphi,
$$

onde $N$ é um fator de normalização tal que $\int_{-\infty}^{\infty} I(\omega) d \omega=1$. Isto significa que será proporcional a área entre as curvas $\omega=\omega_{i n i}$ e $\omega_{f i n}$ na superfície esférica (orientaçōes de $\sigma$ entre o ângulos sólidos $\Omega$ e $\Omega+d \Omega$ ).

Se $p(\Omega) d \Omega$ for a probabilidade de ocorrência das tais orientaçōes de $\sigma$, correspondente a $\omega$ na variação de $\omega$ e $\omega+d \Omega$, teremos:

$$
p(\Omega) d \Omega=I(\omega) d \omega
$$

lembrando que numa amostra em pó todos os ângulos $\Omega$ são igualmente prováveis, resulta para (1.125):

$$
I(\omega)=\frac{1}{4 \pi} \frac{d \Omega}{d \omega}
$$

No caso de $\sigma$ ser axialmente simétrico $(\eta=0)$, temos a dependência de $\omega$ somente do ângulo $\theta$, resultando para (1.124) e conseqüentemente para (1.125), integrada de 0 à $\frac{\pi}{2}$ e $N=\frac{2}{\pi}$ :

$$
\begin{aligned}
\omega & =\frac{\omega_{0} \delta}{2}\left(3 \cos ^{2} \theta-1\right) \\
\int_{\omega_{i}}^{\omega_{f}} I(\omega) d \omega & =\sqrt{\frac{1}{3 \omega_{0} \delta}} \int_{\omega_{i}}^{\omega_{f}} \frac{d \omega}{\left[1+\left(\frac{2 \omega}{\omega_{0} \delta}\right)\right]^{\frac{1}{2}}}
\end{aligned}
$$

Resultando para $I(\omega)$, conforme apresentado na figura (1.18-a)

$$
I(\omega)=\frac{1}{\sqrt{3 \omega_{0} \delta\left[1+2\left(\frac{\omega}{\left.\omega_{0} \delta\right)}\right)\right]}} \text { onde: }-\omega_{0} \frac{\delta}{2} \leq \omega \leq \omega_{0} \delta
$$



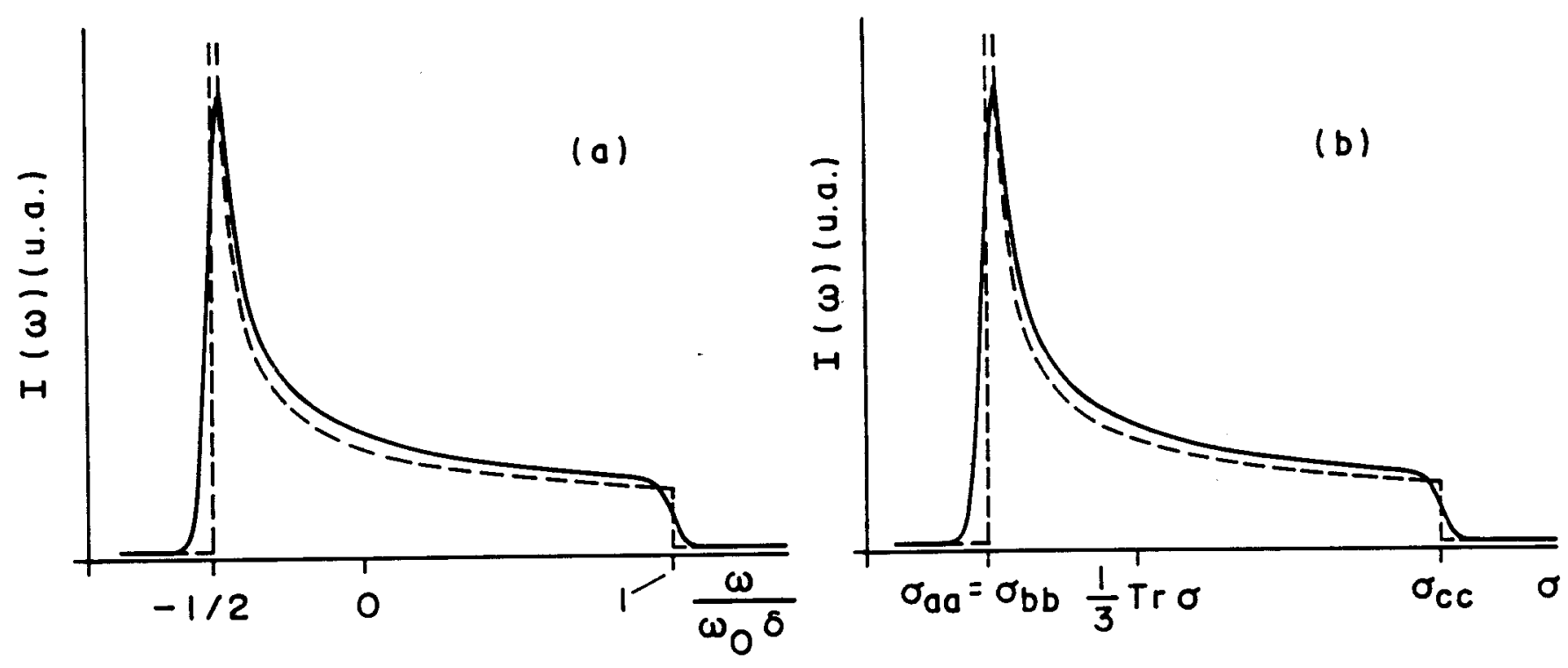

Figura 1.18: Forma da linha de absorção para uma amostra em pó com tensor de deslocamento químico com simetria axial.

Paralelamente, vimos de (1.99), no caso axial que:

$$
\begin{aligned}
\sigma_{z z} & =\left(\sigma_{\|}-\sigma_{\perp}\right) \cos ^{2} \theta+\sigma_{\perp}, \\
\omega_{z z} & =\left(\omega_{\|}-\omega_{\perp}\right) \cos ^{2} \theta+\omega_{\perp},
\end{aligned}
$$

resultando de (1.127), conforme apresentado na figura (1.18-b):

$$
I(\omega)=\frac{1}{4}\left[\left(\omega_{\|}-\omega_{\perp}\right)\left(\omega-\omega_{\perp}\right)\right]^{\frac{1}{2}}
$$

Aqui cabe uma análise da variação de $\omega$, através de (1.128):

- $\omega \rightarrow-\frac{1}{2} \omega_{0} \delta \Rightarrow \theta \rightarrow \frac{\pi}{2}$ e de (1.123) temos $\frac{\omega^{\text {ref }}-\omega}{\omega_{0}}=\sigma_{\perp}$,

- $\omega \rightarrow \omega_{0} \delta \Rightarrow \theta \rightarrow 0$ e de (1.123) temos $\frac{\omega^{r e f}-\omega}{\omega_{0}}=\sigma_{\| 2}$

- $\omega \rightarrow 0 \Rightarrow \cos ^{2} \theta=\frac{1}{3}$ e de (1.123) temos $\frac{\omega^{\text {ref }}-\omega}{\omega_{0}}=\sigma^{i}$.

Tais resultados também podem ser obtidos de (1.133) e são representados na figura (1.18). Observe que tais resultados são os mesmos obtidos na seção anterior e representados na figura (1.16).

No caso de uma simetria qualquer temos $\eta \neq 0$. Utilizando-nos do método de Haeberlen, [2], pag.27, onde é definido:

$$
\omega_{1}=-\frac{1}{2}\left(\omega_{0} \delta\right)(1+\eta)=\omega_{0} \sigma_{a a}
$$




$$
\begin{aligned}
& \omega_{2}=-\frac{1}{2}\left(\omega_{0} \delta\right)(1-\eta)=\omega_{0} \sigma_{b b}, \\
& \omega_{3}=\omega_{0} \delta .
\end{aligned}
$$

A intensidade $I(\omega)$ do espectro de RMN como função de $\omega$ e dado por:

$$
\begin{aligned}
I(\omega) & =\pi^{-1}\left[\left(\omega_{3}-\omega_{2}\right)\left(\omega-\omega_{1}\right)\right]^{-\frac{1}{2}} F\left\{\left[\frac{\left(\omega_{3}-\omega\right)\left(\omega_{2}-\omega_{1}\right)}{\left(\omega_{3}-\omega_{2}\right)\left(\omega-\omega_{1}\right)}\right]^{\frac{1}{2}}, \frac{\pi}{2}\right\} \\
& =\pi^{-1}\left[\left(\omega_{3}-\omega_{2}\right)\left(\omega-\omega_{1}\right)\right]^{-\frac{1}{2}} K\left\{\arcsin \left[\frac{\left(\omega_{3}-\omega\right)\left(\omega_{2}-\omega_{1}\right)}{\left(\omega_{3}-\omega_{2}\right)\left(\omega-\omega_{1}\right)}\right]^{\frac{1}{2}}\right\}
\end{aligned}
$$

para $\omega_{2} \leq \omega \leq \omega_{3}$. Por sua vez para $\omega_{1} \leq \omega \leq \omega_{2}$, temos:

$$
\begin{aligned}
I(\omega) & =\pi^{-1}\left[\left(\omega_{3}-\omega\right)\left(\omega_{2}-\omega_{1}\right)\right]^{-\frac{1}{2}} F\left\{\left(\frac{\left(\omega_{3}-\omega_{2}\right)\left(\omega-\omega_{1}\right)}{\left(\omega_{3}-\omega\right)\left(\omega_{2}-\omega_{1}\right)}\right]^{\frac{1}{2}}, \frac{\pi}{2}\right. \\
& =\pi^{-1}\left[\left(\omega_{3}-\omega\right)\left(\omega_{2}-\omega_{1}\right)\right]^{-\frac{1}{2}} K\left\{\arcsin \left[\frac{\left(\omega_{3}-\omega_{2}\right)\left(\omega-\omega_{1}\right)}{\left(\omega_{3}-\omega\right)\left(\omega_{2}-\omega_{1}\right)}\right]^{\frac{1}{2}}\right\}
\end{aligned}
$$

$F(k, \phi)$ é uma integral eliptica (incompleta) de primeira ordem, e $K(\arcsin k)=$ $F\left(k, \frac{\pi}{2}\right)$ e a integral eliptica completa de primeira ordem. Normalizando-se $I(\omega)$ de maneira que a área sob $I(\omega)$ seja unitária, resulta num espectro de pó apresentado pela figura (1.19).

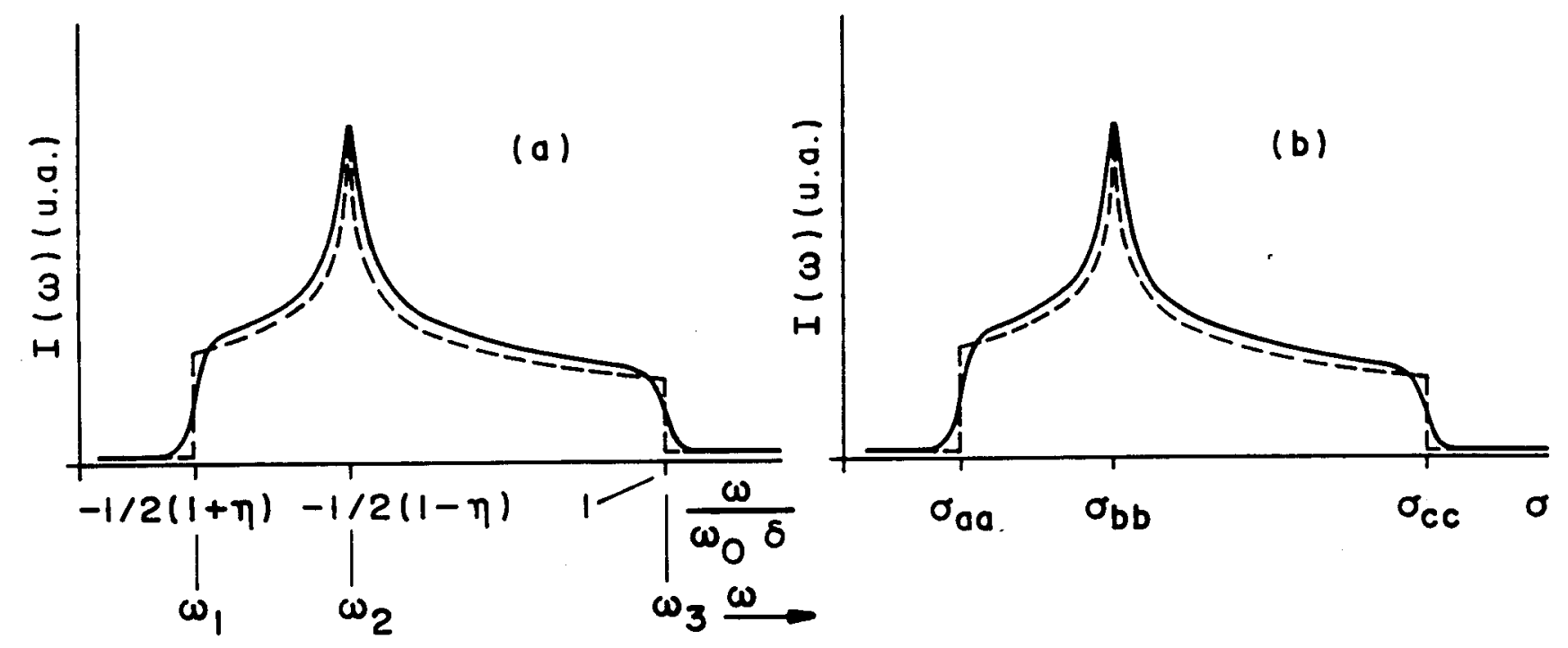

Figura 1.19: Forma de linha de absorção para uma amostra em pó, com tensor de deslocamento químico sem simetria axial $\left(\eta=\frac{2}{3}\right)$.

Observe que as descontinuidades $\omega_{3}, \omega_{2}, \omega_{1}$ resultam em $\sigma_{c c}, \sigma_{b b}, \sigma_{a a}$ ou $\delta, \eta, \sigma$ conforme (1.134), (1.135) e (1.136). Tais componentes podem ser obtidas desses espectros, porém o mesmo não acontece com as orientações dos eixos principais de $\sigma$ com relação aos eixos cristalográficos. 


\subsubsection{Acoplamento Dipolar de um Sistema AX}

Para um sistema heteronuclear de dois núcleos de spin (AX), onde ignoramos quaisquer outras interações, é possível escrever o hamiltoniano de spin como a soma do termo de Zeeman e dipolar truncada no termo A:

$$
\mathcal{H}_{A X}=-\hbar \mathbf{B}_{0}\left(\gamma_{a} \mathbf{I}_{A z}+\gamma_{X} \mathbf{I}_{X z}\right)+\mathcal{H}_{D, A X}
$$

onde:

$$
\mathcal{H}_{D, A X}=-\frac{\hbar^{2} \gamma_{A} \gamma_{X}}{r_{A X}^{3}}\left(3 \cos ^{2} \theta-1\right) \mathbf{I}_{A z} \mathbf{I}_{X z}
$$

No caso de um monocristal, com um só valor para $\mathbf{r}_{A X}$, a ressonância do núcleo A apresenta-se desdobrada em duas linhas simétricas com relação a $\omega_{0 A}=\gamma_{A} B_{0}$ cujas freqüências são:

$$
\omega= \pm \frac{1}{2} R\left(3 \cos ^{2} \theta-1\right)
$$

isso para o núcleo $\mathrm{A}$, sendo que existe também duas linhas para o núcleo $\mathrm{X}, R=\frac{\hbar_{\mathrm{A}}{ }_{\mathrm{Ax}}}{r_{A X}^{\mathrm{J}}}$ é dita constante de acoplamento dipolar entre os spins A e X. Isso lembra (1.128), discutido anteriormente para o caso de simetria axial, $\eta=0$, que resulta no espectro da figura (1.18) para o núcleo $\mathrm{A}$ e a situaçāo semelhante para o núcleo $\mathrm{X}$. $\mathrm{O}$ espectro total constituído pela sobreposição dos espectros dos estados $m_{X}=+\frac{1}{2}$ e $m_{X}=-\frac{1}{2}$, é apresentado na figura (1.20), sendo o resultado a área sombreada.

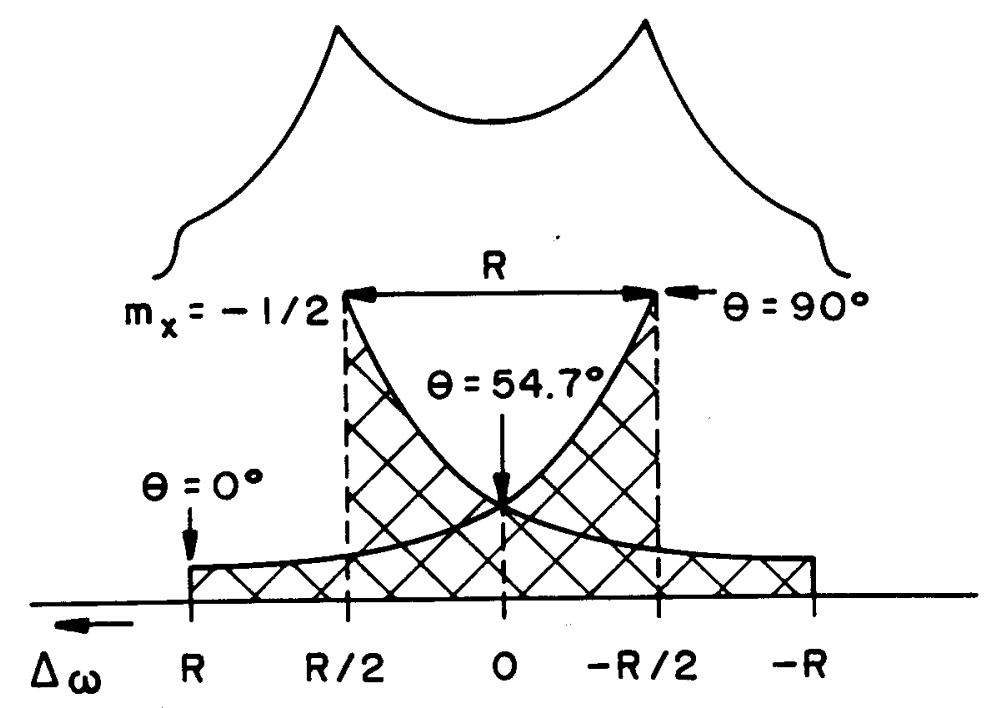

Figura 1.20: Esquema de um espectro de pó, proveniente dum acoplamento dipolar, para $o$ núcleo $A$ num sistema heteronuclear de dois spins $A X$. Indicam-se valores de $\theta$ para $o$ subespectro $m_{X}=+\frac{1}{2}$. 
Observe que este espectro permite obter o valor de $R$ variando-se os valores de $\theta$, conforme fizemos no caso de deslocamento químico, uma vez obtido o valor de $R$ podemos obter o valor de $r$. Essa separação foi obtida anteriormente (1.51) onde analisamos um sistema heteronuclear, a separação $4 \Omega$ que obtivemos nada mais é que $R$, pois $4 \Omega=R$, conforme figuras $(1.20) \mathrm{e}(1.5-\mathrm{b})$.

No caso de um sistema com acoplamento dipolar homonuclear, vimos de (1.72), (1.73), (1.74), (1.75) o aparecimento de dos estados tripleto $|1 M\rangle$ e singleto $|00\rangle$. As possíveis transições correspondem a freqüência de $\omega+3 \Omega$ e $\omega-3 \Omega$, que resulta no dubleto centrado em $\omega$ com separação $6 \Omega$, isto é $\frac{3}{2} D$ conforme figuras $(1.21)$ e $(1.7-b)$.

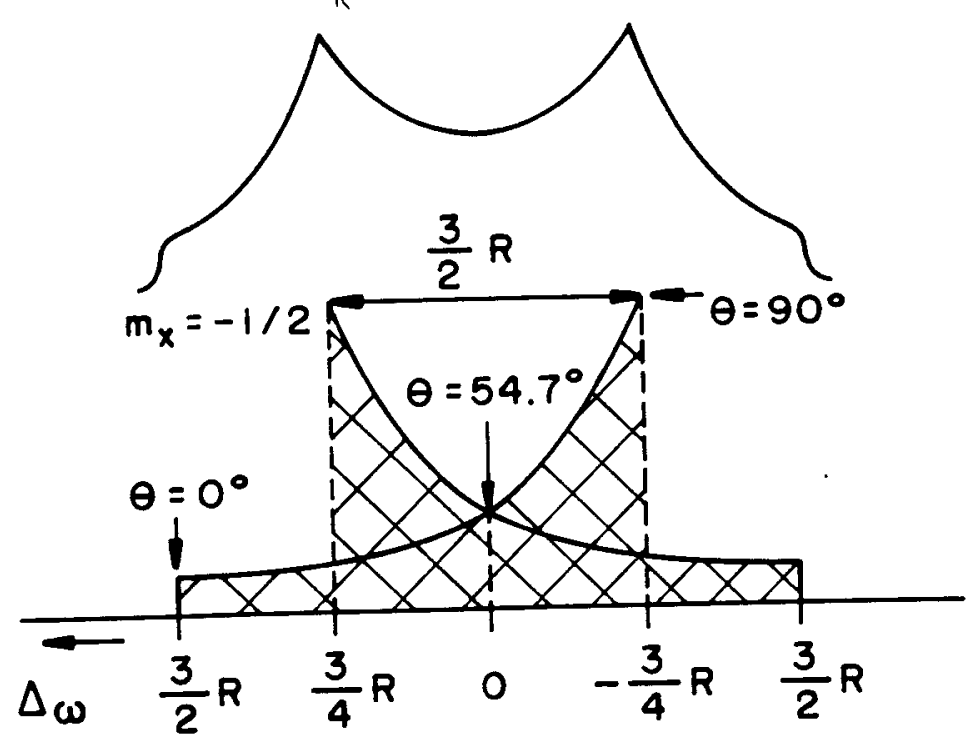

Figura 1.21: Esquema de um espectro de pó, proveniente dum acoplamento dipolar, para $o$ núcleo $A$ num sistema homonuclear de dois spins $A X$. Indicam-se valores de $\theta$ para $o$ subespectro $m_{X}=+\frac{1}{2}$. 
38 CAP'TULO 1. HAMILTONIANO DE SPIN NUCLEAR EM SÓLIDOS 


\section{Bibliografia}

[1] M. E. Rose, Elementary Theory of Angular Momentum, (1967), J. Wiley.

[2] U. Haeberlen, High Resolutions NMR in Solids, (1976), Academic Press.

[3] C. Cohen-Tannoudji, B. Diu, F. Laloë, Mécanique Quantique, vol.2, (1977), Hermann.

[4] C. P. Slichter, Principles of Magnetic Resonance, (1980), Spring-Verlag, 2nd edition.

[5] M. Mehring, Principles of High Resolution NMR in Solids (1983), Spring-Verlag.

[6] N. Chandrakumar, S. Subramanian, Modern Techniques in High Resolution FT-NMR (1987), Spring-Verlag.

[7] V. M. S. Gil, C. F. Geraldes, Ressonância Magnética Nuclear, (1987), F. Calouste Gulbenkian.

[8] R. K. Harris, Nuclear Magnetic Resonance Spectroscopy, A Physicochemical View, (1986), Longman.

[9] R. G. Griffin, in International School of Physics "Enrico Fermi", Societá Italiana di Fisica, Varenna (1986) - 2nd Course.

[10] E. R. Andrew, in Philosophical Transactions of The Royal Society of London - Mathematical and Physical Sciences - Nuclear Magnetic Resonance Spectroscopy in Solids, 299, 621-641, No 1452, (1981). 
BIBLIOGRAFIA 


\section{Capítulo 2}

\section{Rotação da amostra em Torno do Ângulo Mágico}

\subsection{Introdução}

Tratando-se de amostras sólidas, uma característica básica de seu comportamento são os largos espectros de RMN, quando comparados com uma amostra líquida.

Tal diferença, conforme discutimos no capitulo 1, reside nas interações anisotrópicas estáticas a que um núcleo, presente numa amostra sólida, está sujeito. Numa amostra líquida, naturalmente temos os movimentos aleatórios isotrópicos e rápidos dos núcleos que resulta num processo de média dos tensores de segunda ordem responsáveis pelas interações anisotrópicas, removendo-as efetivamente do espectro de RMN.

Dependendo da amostra sólida podemos encontrar tais movimentos nucleares, o suficiente para estreitar o espectro de RMN e, às vezes suficiente o bastante para resolvermos tal espectro. Isso é o caso por exemplo de núcleos $P^{31}$, numa amostra policristalina $P_{4} S_{3}$, numa temperatura de $420 \mathrm{~K}, 26 \mathrm{~K}$ abaixo do seu ponto de fusão, conforme Andrew [5], [9].

Porém, nos casos mais gerais, nāo encontramos esse movimento suficiente para estreitar os espectros de RMN. Nessa situação podemos emular esse movimento impondo-o ao núcleo. Isso foi feito inicialmente girando rapidamente uma amostra sólida, Andrew [1] e Lowe [2]. Poucos anos depois, uma maneira alternativa foi sugerida impondo agora, o movimento do núcleo no espaço de spin, Mansfield $\mathscr{G}$ Ware [3] e Ostroff $\mathscr{G}$ Waugh [4].

Em particular para materiais policristalinos e amorfos, nossos materiais de maior interesse, o estreitamento do espectro só pode ser obtido com a utilização da Técnica de Rotação da amostra em torno do Ângulo Mágico, que remove as fontes anisotrópicas de alargamento de linha.

Discutiremos nesse trabalho, Técnica de Rotação da amostra em torno do Ângulo Mágico para sistemas nucleares de spin $1 / 2$ onde as interações que prevalecem são as interaçōes dipolares e deslocamento químico anisotrópico. 


\subsection{Rotações e Sistemas de Referências}

Inicialmente, analisamos tal efeito sobre a interação dipolar que está sempre presente numa amostra sólida e foi historicamente a primeira a ser considerada e removida. Como vimos, seu Hamiltoniano para todos os pares nucleares $i$ e $k$ no sólido, é dado por:

$$
\mathcal{H}_{D}=-2 \hbar \sum_{i<k} \gamma_{n}^{i} \gamma_{n}^{k} \sum_{m=-2}^{+2} R_{2,-m}^{D, i k} T_{2, m}^{D, i k}
$$

Precisamos agora transformar o termo espacial $R_{2,-m}^{D, i k}$ para cada par de núcleos para o referencial do laboratório em termos de suas componentes num referencial da amostra, este por sua vez girando no referencial do laboratório. Posteriormente, essas componentes do referencial da amostra são expressas em termos de quantidades invariantes $\vartheta_{2 m}^{D, i k}$ do tensor no seu sistema de eixos principais. Isso é feito conforme o procedimento que fizemos no capítulo 1 para o deslocamento químico, onde vimos que essas duas transformações são convenientemente acopladas pelas matrizes de rotação de Wigner $\mathcal{D}_{m^{\prime} m}^{\ell}\left(\Omega^{\prime}\right)$ e $\mathcal{D}_{m^{\prime} m}^{\ell}\left(\Omega^{\prime \prime}\right)$, conforme (1.17) resultando:

$$
\begin{aligned}
R_{2,-m}^{D, i k}(L A B) & =\sum_{m^{\prime}} \mathcal{D}_{m^{\prime},-m}^{(2)}\left(\Omega^{\prime \prime}\right) R_{2,-m}^{D, i k}(A M O) \\
& =\sum_{m^{\prime}} \mathcal{D}_{m^{\prime},-m}^{(2)}\left(\Omega^{\prime \prime}\right) \sum_{m^{\prime \prime}} \mathcal{D}_{m^{\prime \prime}, m^{\prime}}^{(2)}\left(\Omega_{i k}^{\prime}\right) \vartheta_{2 m^{\prime \prime}}^{D, i k}
\end{aligned}
$$

onde:

- $\vartheta_{2 m}^{D, i k}$ são quantidades invariantes sob rotação.

- os ângulos de Euler $\Omega_{i k}^{\prime}=\left(\alpha_{i k}^{\prime}, \beta_{i k}^{\prime}, \gamma_{i k}^{\prime}\right)$ relacionam o referencial da amostra $(\hat{\imath}, \hat{j}, \hat{k})$ arbitrariamente escolhido com o referencial dos eixos principais $(\hat{a}, \hat{b}, \hat{c})$; observe que $\Omega_{i k}^{\prime}$ é diferente para diferentes pares de spin.

- $\Omega "=(\alpha ", \beta ", \gamma ")$ é um conjunto de ângulos de Euler que relaciona o sistema do laboratório $(\hat{x}, \hat{y}, \hat{z})$ com os eixos da amostra $(\hat{\imath}, \hat{j}, \hat{\mathbf{k}})$.

Girando a amostra de $\omega_{\tau}$ em torno de um eixo $\hat{k}$ inclinado po um ângulo arbitrário $\beta "=\theta$ com relação ao campo aplicado $B_{0}$, resulta que $\Omega$ " passa a ser dependente do tempo, conforme figura (2.1).

$$
\Omega "=\Omega "\left(0, \beta ", \omega_{r} t\right) .
$$

Como o campo aplicado excede por várias ordens de magnitude o campo dipolar interno, somente as componentes que sāo paralelas ou anti-paralelas ao campo aplicado são mais importantes quando somados ao campo aplicado. Assim "truncamos" ou restringimo-nos aos "termos seculares", $m=0$, do Hamiltoniano Dipolar.

A contribuição de campo de um núcleo vizinho, seja paralelo ou anti-paralelo ao campo aplicado, depende entre outras coisas da orientação de spin desse núcleo. Normalmente 


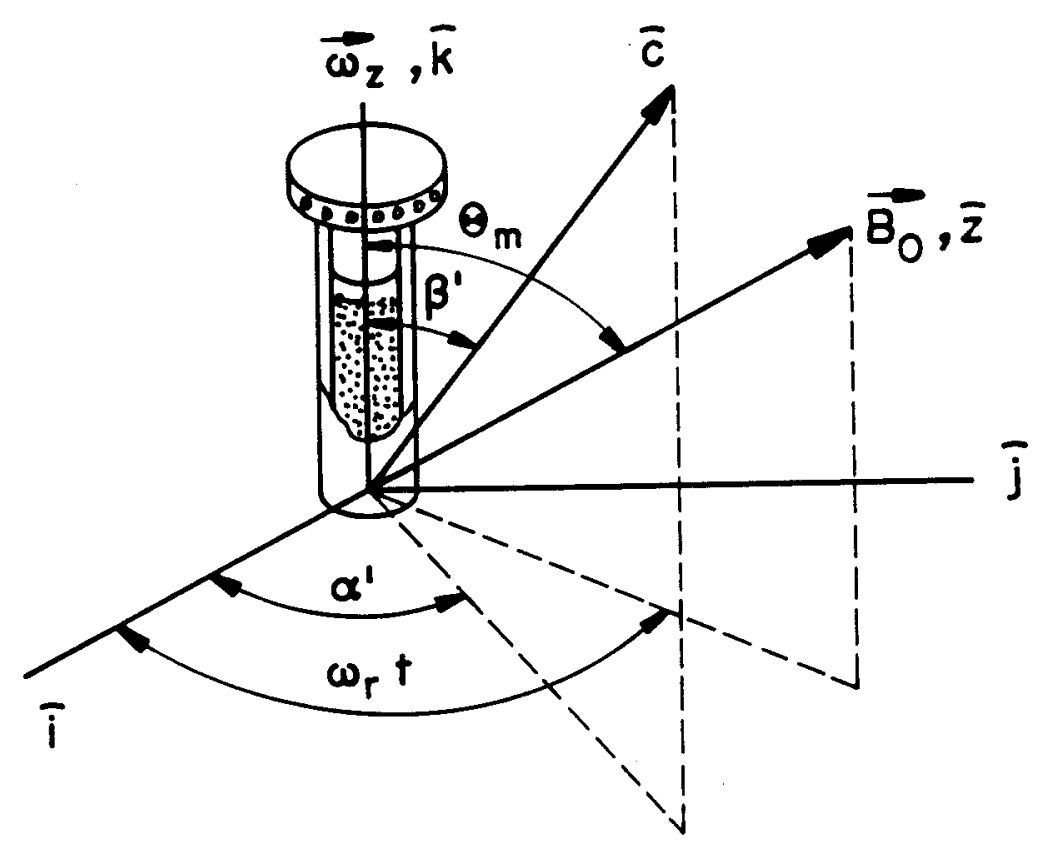

Figura 2.1: Relação entre os sistema de referência da amostra $(\hat{i}, \hat{j}, \hat{k})$ com o sistema de eixos principais $(\hat{a}, \hat{b}, \hat{c})$ e o sistema do campo magnético externo $\mathbf{B}_{\mathbf{0}},(\hat{x}, \hat{y}, \hat{z})$.

os spins são orientados em sua boa parte aleatoriamente. Portanto, o campo dipolar que D spin nuclear "vê" é uma soma de contribuições de sinais que podem ser tanto $(+)$ como $(-)$, resultando que para um conjunto de spins numa amostra macroscópica, existe uma distribuição de magnitudes de campo magnético, que reflete num espectro da amostra com alargamento de linha dipolar.

Assim para os termos seculares, temos:

$$
\mathcal{D}_{00}^{2}\left(0, \beta^{\prime \prime}, \omega_{r} t\right)=\frac{1}{2}\left(3 \cos ^{2} \beta^{\prime \prime}-1\right),
$$

por sua vez, sendo $\vartheta_{2 m^{\prime \prime}}^{D, i k}=0$; para $m^{\prime \prime} \neq 0$, conforme $(1.26),(1.25)$ e $(1.27)$, resulta:

$$
R_{2,0}^{D, i k}=\sqrt{\frac{3}{2}} \frac{1}{r_{i k}^{3}} \mathcal{D}_{00}^{2}\left(0, \beta^{\prime \prime}, \omega_{r} t\right) \mathcal{D}_{00}^{2}\left(\Omega_{i k}^{\prime}\right) .
$$

Se girarmos a amostra segundo um eixo de rotação inclinado de um ângulo $\beta "=\beta_{m}=$ $\arccos \left(\frac{1}{\sqrt{3}}\right)=54^{\circ} 44^{\prime}$ dito Ângulo Mágico, as interações descritas pelo tensor de segunda ordem $\mathcal{D}_{00}^{2}\left(0, \beta ", \omega_{\tau} t\right)$, que usualmente governam as anisotropias dos espectros de RMN, serão mediadas a zero. Isto foi feito experimentalmente e independentemente por Andrew [1] e Lowe [2].

Observe que, se todos os tensores da amostra possuirem simetria axial cilíndrica, quando rodamos a amostra suficientemente rápido, todos os tensores e todos os vetores que conectam dois spin nucleares distintos, estarão girando sobre cones diversos, resultando em média um único eixo de rotação na mesma direção do eixo de rotação da amostra, 
conforme figura (2.2). Eliminamos assim anisotropias não só do tipo interação ${ }^{1}$ dipolar, mas também deslocamentos químicos, que sob rotação espacial transformam-se da mesma maneira que a interação dipolar, $(2.5)$, tornando-se isotró:ico $\left(\mathcal{H}_{D Q}=\omega_{0} I_{0} \sigma\right)$, como de uma amostra em solução.

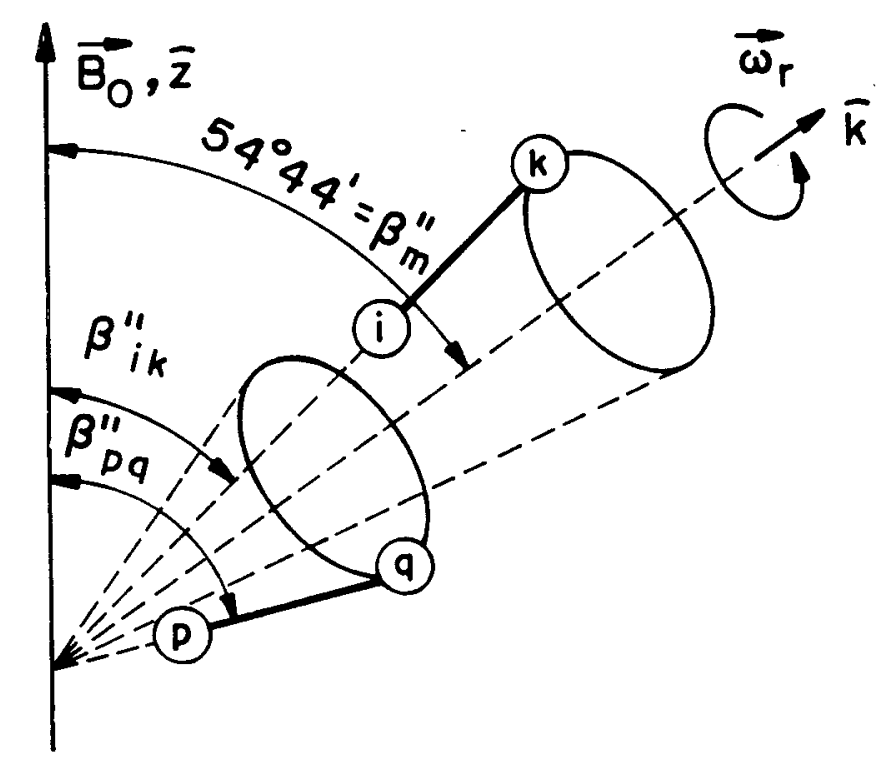

Figura 2.2: Rotação da amostra em torno do ângulo mágico, resultando para os valores médios $\left\langle\beta^{\prime \prime}{ }_{i k}\right\rangle=\left\langle\beta_{p q}\right\rangle=\arccos \left(\frac{1}{\sqrt{3}}\right)$, sendo que $\beta^{\prime \prime}{ }_{i k} \neq \beta^{\prime \prime}{ }_{p q}$.

Porém, para mediar efetivamente todas as interaçōes de spin precisamos girar a amostra numa frequência da ordem das interações entre os spins nucleares. Por exemplo para um acoplamento dipolar de $2.5 \mathrm{kHz}$, precisamos girar a amostra numa freqüência maior que 2500 rotações por segundo, para que possamos nos livrar efetivamente dessa interação.

\subsection{Ecos Rotacionais e Bandas Laterais}

A situação descrita anteriormente nem sempre ocorre, existem situaçōes onde a rotação da amostra é menor que as freqüências das interações presentes, isto é, a rotação não é rápida o suficiente para remover tais interaçoos. Nesses casos surgem os Ecos Rotacionais no domínio do tempo e as Bandas Laterais no domínio da frequiência.

Para entendermos tais fenômenos, consideremos uma interação de spin nuclear dada no referencial do laboratório por:

$$
\mathcal{H}_{\lambda}=C^{\lambda} \sum_{\ell=0}^{2} \sum_{m=-\ell}^{+\ell}(-1)^{m} R_{\ell,-m}^{\lambda}(t) T_{\ell m}^{\lambda}\left(t^{\prime}\right)
$$

\footnotetext{
${ }^{1}$ Podemos citar aqui também a interação quadrupolar, que não é do nosso interesse nesse trabalho.
} 
Observe que as dependências temporais são diferentes: o tempo $t^{\prime}$ em $T_{\ell m}^{\lambda}\left(t^{\prime}\right)$ provém da precessão $\omega_{0}$ do spin no campo magnético estático, enquanto que a dependência $t$ em $R_{\ell,-m}^{\lambda}(t)$ é imposta pela rotação da amostra. Para os termos seculares $(m=0)$, resulta:

$$
\mathcal{H}_{S}=C^{\lambda}\left\{R_{00} T_{00}+R_{10}(t) T_{10}\left(t^{\prime}\right)+R_{20}(t) T_{20}\left(t^{\prime}\right)\right\}
$$

De $(1.8),(1.9)$ e (1.10) vimos que para um tensor simétrico, a componente $R_{10}$ não contribui para interação sendo $R_{\ell, m} \neq 0$ para $\ell=0$ e $\ell=2$. Por sua vez a parte isotrópica $R_{00}=-\frac{1}{\sqrt{3}} \operatorname{Tr}\left\{R_{i j}\right\}$ é invariante por rotação, logo:

$$
\mathcal{H}_{s}=C^{\lambda}\left\{R_{00} T_{00}+R_{20}(t) T_{20}\left(t^{\prime}\right)\right\}
$$

Para $R_{20}(t)$ no referencial do laboratório temos:

$$
R_{20}(t)=\sum_{m^{\prime}=-2}^{+2} \mathcal{D}_{m^{\prime}, 0}^{2}\left(\Omega^{\prime \prime}\right) R_{2 m^{\prime}}(A M O),
$$

onde $\Omega$ " carrega a dependência temporal pois $\Omega "=\left(0, \theta, \omega_{\tau} t\right)$. Utilizando-se das matrizes de rotação de Wigner, resulta:

$$
\begin{aligned}
\mathcal{H}_{s}= & C^{\lambda}\left\{R_{00} T_{00}+T_{20} \sum_{m^{\prime}} \mathcal{D}_{m^{\prime} 0}^{2}\left(0, \theta, \omega_{r} t\right) R_{2 m^{\prime}}(A M O)\right\} \\
= & C^{\lambda}\left\{R_{00}(A M O) T_{00}+T_{20}\left[\frac{1}{2}\left(3 \cos ^{2} \theta-1\right) R_{20}(A M O)\right.\right. \\
& +\sqrt{\frac{3}{8}} \sin 2 \theta\left(R_{21}(A M O) \exp \left(i \omega_{r} t\right)-R_{2,-1}(A M O) \exp \left(-i \omega_{r} t\right)\right) \\
& \left.+\sqrt{\frac{3}{8}} \sin ^{2} \theta\left(R_{22}(A M O) \exp \left(2 i \omega_{r} t\right)+R_{2,-2}(A M O) \exp \left(-2 i \omega_{r} t\right)\right)\right]
\end{aligned}
$$

Sendo que $R_{2 m}(A M O)$ pode ser diagonalizado, devido a uma rotação $\Omega^{\prime}=\left(\alpha^{\prime}, \beta^{\prime}, \gamma^{\prime}\right)$ que leva o sistema da amostra para o sistema de eixos principais, resultando para as componentes do tensor:

$$
R_{2 m}(A M O)=\sum_{m^{\prime}=-2}^{+2} \vartheta_{2 m^{\prime}} \exp \frac{\left(-i m^{\prime} \alpha^{\prime}\right)}{\uparrow !} \mathcal{D}_{m^{\prime}, m}^{2}\left(\beta^{\prime}\right) \frac{\exp \left(-i m \gamma^{\prime}\right)}{4}
$$

onde $\left(\alpha^{\prime}, \beta^{\prime}, \gamma^{\prime}\right)$ são os ângulos de Euler que relacionam os eixos principajs com o sistema dos eixos do rotor (AMO), conforme figuras (2.1) $\mathscr{E}(1.17)$.

Vimos que para o sistema de eixos principais, conforme $(1.8),(1.9)$ e $(1.10)$, resulta:

$$
\begin{aligned}
\vartheta_{2,0} & =\sqrt{\frac{3}{2}}\left(\delta_{c c}-\delta\right)=\sqrt{\frac{3}{2}} \delta \\
\vartheta_{2, \pm 1} & =0 \\
\vartheta_{2, \pm 2} & =\sqrt{\frac{3}{2}}\left(\delta_{a a}-\delta_{b b}\right)=\frac{1}{2} \delta \eta .
\end{aligned}
$$


Utilizando-se agora desse resultado, vem de (2.10);

$$
\begin{aligned}
R_{20}(t)= & \sum_{m}\left\{\sum_{m^{\prime}} \vartheta_{2, m^{\prime}} \exp \left(-i m^{\prime} \alpha^{\prime}\right) \mathcal{D}_{m^{\prime}, m}^{2}\left(\beta^{\prime}\right) \exp \left(-i m \gamma^{\prime}\right)\right\} \\
& \times \exp \left(-i m \omega_{r} t\right) \mathcal{D}_{m^{\prime}, 0}^{2}(\theta)
\end{aligned}
$$

Realizando-se as somatórias e substituindo-se os valores de $\vartheta_{2 m^{\prime}}$, lembrando-se também que a parte isotrópica de $R_{\ell, m}$ é dada por $\frac{1}{\sqrt{3}} \vartheta_{00}=R=\frac{1}{3} \operatorname{Tr} R$, resulta para o Hamiltoniano secular (2.12):

$$
\begin{aligned}
\mathcal{H}_{s}= & C^{\lambda}\left\{R T_{00}+\sqrt{\frac{3}{2}} \delta T_{20}\left[\frac{1}{2}\left(3 \cos ^{2} \theta-1\right)\right]\right. \\
& \times\left[\frac{1}{2}\left(3 \cos ^{2} \beta^{\prime}-1\right)+\frac{\eta}{2} \sin ^{2} \beta^{\prime} \cos 2 \gamma^{\prime}\right]+\sqrt{\frac{3}{2}} \delta T_{20} \Lambda(t),
\end{aligned}
$$

onde:

$$
\Lambda(t)=C_{1} \cos \omega_{r} t+S_{1} \sin \omega_{r} t+C_{2} \cos 2 \omega_{r} t+S_{2} \sin 2 \omega_{r} t
$$

e por sua vez:

$$
\begin{aligned}
C_{1}= & \frac{1}{2} \sin 2 \theta \sin \beta^{\prime}\left[\cos \beta^{\prime}\left(\eta \cos 2 \gamma^{\prime}-3\right) \cos \alpha^{\prime}-\eta \sin 2 \gamma^{\prime} \sin \alpha^{\prime}\right] \\
S_{1}= & \frac{1}{2} \sin 2 \theta \sin \beta^{\prime}\left[\cos \beta^{\prime}\left(3-\eta \cos 2 \gamma^{\prime}\right) \sin \alpha^{\prime}-\eta \sin 2 \gamma^{\prime} \cos \alpha^{\prime}\right] \\
C_{2}= & \frac{1}{2} \sin ^{2} \theta\left\{\left[\frac{3}{2} \sin ^{2} \beta^{\prime}+\frac{\eta}{2} \cos 2 \gamma^{\prime}\left(1+\cos ^{2} \beta^{\prime}\right)\right] \cos 2 \alpha^{\prime}\right. \\
& \left.-\eta \cos \beta^{\prime} \sin 2 \gamma^{\prime} \sin 2 \alpha^{\prime}\right\} \\
S_{2}= & \frac{1}{2} \sin ^{2} \theta\left\{-\left[\frac{3}{2} \sin ^{2} \beta^{\prime}+\frac{\eta}{2} \cos 2 \gamma^{\prime}\left(1+\cos ^{2} \beta^{\prime}\right)\right] \sin 2 \alpha^{\prime}\right. \\
& \left.-\eta \cos \beta^{\prime} \sin 2 \gamma^{\prime} \cos 2 \alpha^{\prime}\right\}
\end{aligned}
$$

Agora considerando-se o deslocamento químico anisotrópico, sendo as componentes de $T_{\ell m}$ dadas por:

$$
\begin{aligned}
& T_{00}=\frac{1}{\sqrt{3}} I_{z} B_{o} \\
& T_{20}=\sqrt{\frac{2}{3}} I_{z} B_{o}
\end{aligned}
$$

de (2.18) resulta para um particular grupo $k$ isocromático de spins nucleares:

$$
\begin{aligned}
\mathcal{H}_{S, D Q}= & \gamma_{k} I_{z} B_{0}\left\{\sigma+\delta_{k}\left[\frac{1}{2}\left(3 \cos ^{2} \theta-1\right)\right]\right. \\
& \left.\times\left[\frac{1}{2}\left(3 \cos ^{2} \beta^{\prime}-1\right)+\frac{\eta_{k}}{2} \sin ^{2} \beta^{\prime} \cos 2 \gamma^{\prime}\right]+\delta_{k} \Lambda_{k}(t)\right\}
\end{aligned}
$$


donde podemos obter a freqüência desse grupo de spin $k$, dado por:

$$
\begin{aligned}
\omega(t)= & \omega_{0}\left\{\sigma+\delta_{k}\left[\frac{1}{2}\left(3 \cos ^{2} \theta-1\right)\right]\right. \\
& \left.\times\left[\frac{1}{2}\left(3 \cos ^{2} \beta^{\prime}-1\right)+\frac{\eta_{k}}{2} \sin ^{2} \beta^{\prime} \cos 2 \gamma^{\prime}\right]+\delta_{k} \Lambda_{k}(t)\right\} .
\end{aligned}
$$

Observe que $\omega(t)$ pode ser separada em duas partes:

$$
\begin{aligned}
& \omega(t)=\bar{\omega}+\omega_{0} \delta_{k} \Lambda_{k}(t), \\
& \bar{\omega}=\omega_{0}\left\{\sigma+\delta_{k}\left[\frac{1}{2}\left(3 \cos ^{2} \theta^{\prime}-1\right)\right] \times\left[\frac{1}{2}\left(3 \cos ^{2} \beta^{\prime}-1\right)+\frac{\eta_{k}}{2} \sin ^{2} \beta^{\prime} \cos 2 \gamma^{\prime}\right]\right\} .
\end{aligned}
$$

Ainda considerando-se a interação de deslocamento químico, numa amostra em pó de maneira que na situação estacionária o espectro de cada espécie $n$, distintas quimicamente tenha o espectro de pó característico, conforme a figura (1.18) $\mathcal{B}(1.19)$. Para cada espécie $n$, temos $k$ grupos de spin isocromáticos, resultando na situação de ângulo mágico:

$$
\begin{aligned}
\mathcal{H}_{s e c, D Q}^{k, n} & =\gamma_{n} B_{0} I_{z n}\left[\sigma_{n}+\delta_{n} \Lambda_{k n}(t)\right] \\
& =\omega_{k n}(t) I_{z n}
\end{aligned}
$$

onde:

$$
\omega_{k n}(t)=\omega_{0}\left\{\sigma_{n}+\delta_{n} \Lambda_{k n}\right\}
$$

sendo:

$$
\begin{aligned}
\sigma_{n} & =\frac{1}{3}\left[\sigma_{a a, n}+\sigma_{b b, n}+\sigma_{c c, n}\right], \\
\delta_{n} & =\sigma_{c c, n}-\sigma_{n} .
\end{aligned}
$$

Se a amostra gira lentamente, isto é $\omega_{r} \ll \omega_{0} \delta_{n}$, o FID seguindo um pulso de $\frac{\pi}{2}$ decai rapidamente no tempo $\sim\left(\omega_{0} \delta_{n}\right)^{-1}$, tal como no caso estacionário. Assim cada grupo $k$, tem sua magnetização inicialmente ao longo do eixo $x$ do referencial que gira, resultando no tempo um deslocamento de $\phi_{k n}(t)$, dado por:

$$
\begin{aligned}
\phi_{k n}(t) & =\int_{0}^{t} \omega_{k n}\left(t^{\prime}\right) d t^{\prime} \\
& =\omega_{0} \sigma_{n} t+\omega_{0} \delta_{n} \int_{0}^{t} \Lambda_{k n}\left(t^{\prime}\right) d t^{\prime} .
\end{aligned}
$$

Por sua vez o vetor magnetização relaxa com $\exp \left(-\frac{t}{T_{2}}\right)$, mas ao mesmo tempo ele processa-se sobre o eixo z com uma freqüência de "offset" $\omega_{k n}$. Resulta que a ponta do vetor descreve um decaimento tipo saca-rolha, conforme figura (2.3). Experimentalmente detectamos a projeção de $M$ sobre os planos $y^{\prime}$ e $x^{\prime}$, que são dadas por:

$$
\begin{aligned}
& M_{x^{\prime}}=M_{0} \exp \left(-\frac{t}{T_{2}}\right) \sin \phi_{k n} t, \\
& M_{y^{\prime}}=M_{0} \exp \left(-\frac{t}{T_{2}}\right) \cos \phi_{k n} t .
\end{aligned}
$$




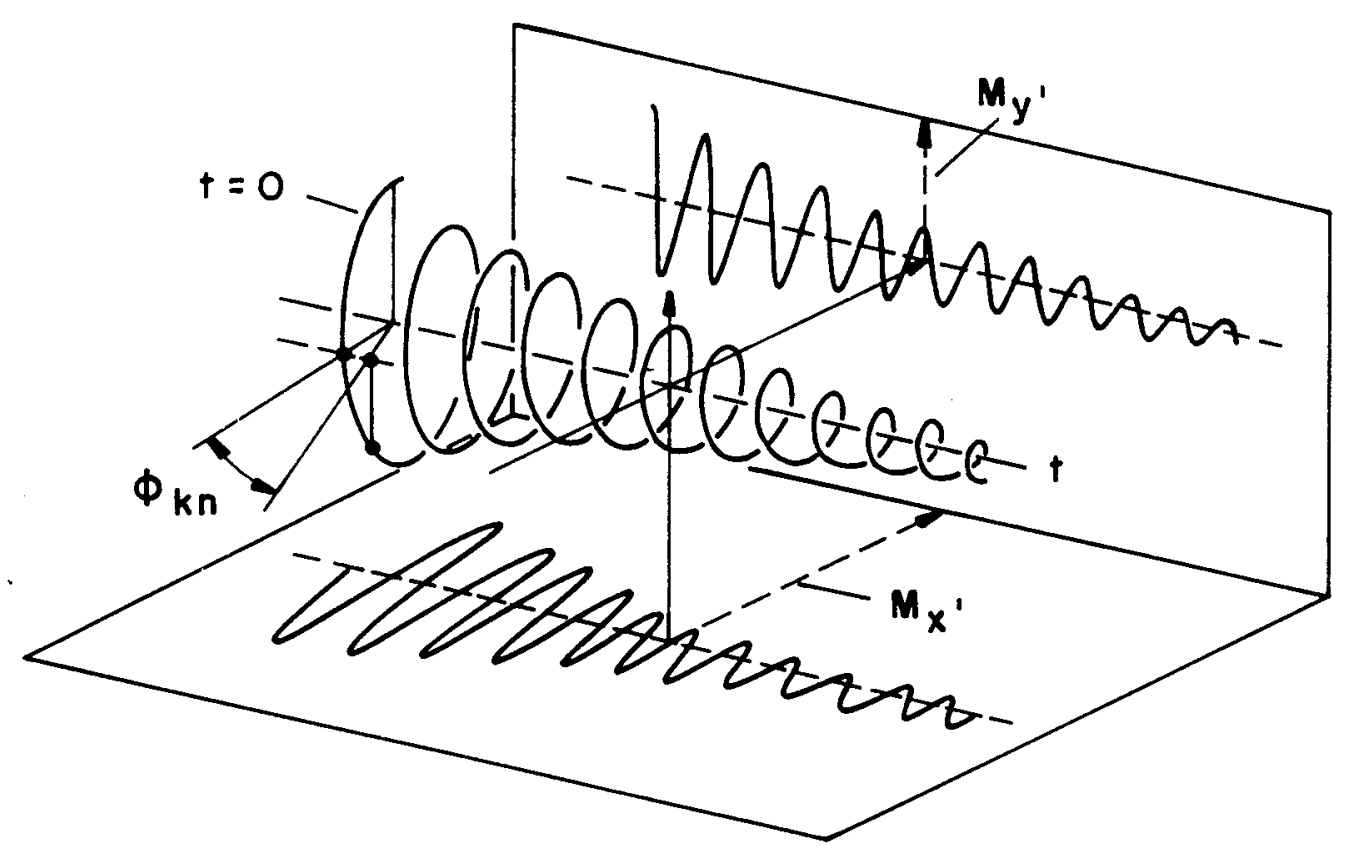

Figura 2.3: Decaimento e precessão da magnetização nuclear, seguido de um pulso, para um determinado grupo isocromático $k$ de spin, pertencente a uma espécie química $n$.

Lembrando que um espectro consiste em mais de uma linha, o FID será a soma de todos esses decaimentos para cada linha, onde teremos $n$ grupos de núcleos com vizinhanças quimicamente distintas e para cada grupo $n$ temos $k$ grupos isocromáticos de spins, representando $k$ linhas distintas, conforme figura (2.4), resultando para o FID:

$$
g(t)=\sum_{n} \sum_{k} p_{n} \exp \left(i \phi_{k n}(t)\right)
$$

onde $p_{n}$ representa a população das espécies $n$ na amostra e exp $\left(i \phi_{k n}(t)\right)$ contém as dependências da magnetização com $\cos \phi_{k n}(t)$ e $\sin \phi_{k n} t$, sendo que o termo $\exp \left(-t / T_{2}\right)$ é desprezível, comparado com t pois $T_{2}$ é muito longo.

Combinando-se (2.39) $\mathcal{G}(2.36)$ resulta:

$$
g(t)=\sum_{n} p_{n} \exp \left(i \omega_{0} \sigma_{n} t\right) \sum_{k} \exp \left[i \omega_{0} \delta_{n} \int_{0}^{t} \Lambda_{k n}\left(t^{\prime}\right) d t^{\prime}\right]
$$

De (2.40) vemos que o fator que precede a soma sobre $k$ representa uma precessão uniforme do azimute no qual os sucessivos ecos são refocados, e é justamente o FID de uma amostra líquida, dado por $g_{I n}(t)$ :

$$
g_{\text {In }}(t)=p_{n} \exp \left(i \omega_{0} \sigma t\right)
$$




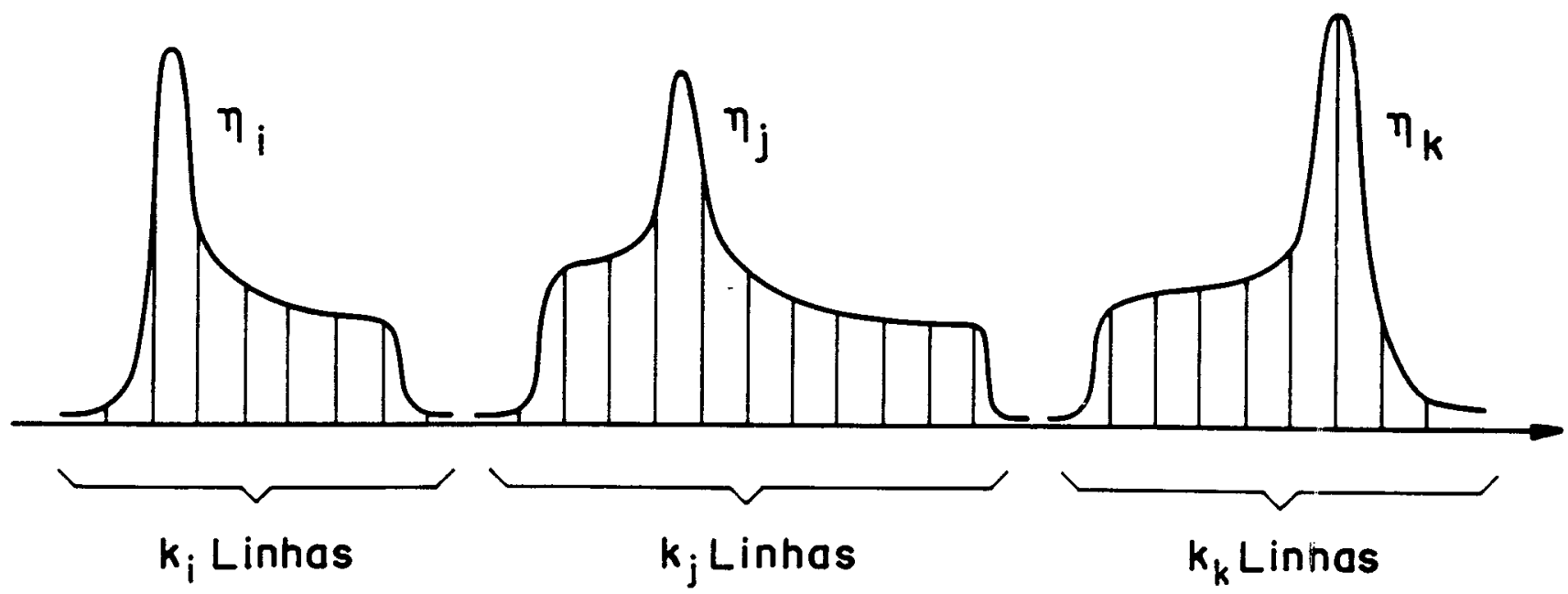

Figura 2.4: Representação de $n$ grupos de spins distintos quimicamente com suas respectivas $k$ linhas isocromáticas.

No que diz respeito a termo somado sobre $k$ e $n$, observe que devido ao fato de $\Lambda_{k n}$ ser puramente oscilatório resulta que a integral se anula para os valores $t=\left(\frac{2 \pi}{\omega_{r}}\right) p$, sendo $p$ inteiro. Essa soma sobre $k$ representa um Trem de Ecos com forma $g_{E n}\left(t-p\left(\frac{2 \pi}{\omega_{r}}\right)\right)$, dado por:

$$
g_{E_{n}}\left(t-p\left(\frac{2 \pi}{\omega_{r}}\right)\right)=\sum_{k} \exp \left(i \omega_{0} \delta_{n} \int_{0}^{t} \Lambda_{k n}\left(t^{\prime}\right) d t^{\prime}\right) .
$$

Agora utilizando-se da função impulso para os vários $p \frac{2 \pi}{\omega_{r}}=t$, dada por:

$$
\Im\left(t-\frac{2 \pi}{\omega_{r}}\right)=\sum_{p=-\infty}^{+\infty} \delta\left(t-p\left(\frac{2 \pi}{\omega_{r}}\right)\right)
$$

e da propriedade de reprodutividade do trem de eco, que é representado pelo produto de convolução de $g_{E} \operatorname{com} \Im\left(t-\frac{2 \pi}{\omega_{r}}\right)$ :

$$
g_{E n}\left(t-p\left(\frac{2 \pi}{\omega_{r}}\right)\right)=\Im\left(t-\frac{2 \pi}{\omega_{r}}\right) \otimes g_{E_{n}}(t),
$$

\footnotetext{
${ }^{2} \mathrm{~A}$ Convolução de duas funções $x(t)$ e $h(t)$ é dada por:

$$
y(t)=x(t) \otimes h(t)=\int_{-\infty}^{+\infty} x(\tau) h(t-\tau) d t
$$

$$
y(t)=x(t) \otimes h(t)=\int_{-\infty}^{+\infty} h(t) x(t-\tau) d t
$$
}

ou

$y(t)$ é dita Convolução de $x(t)$ e $h(t)$. 
50 CAPítulo 2. ROTAÇÃO DA AMOSTRA EM TORNO DO ÁNGULO MÁGICO resulta de (2.44) Ef (2.41):

$$
g(t)=\sum_{n} g_{I n}(t)\left[\Im\left(t-\frac{2 \pi}{\omega_{\tau}}\right) \otimes g_{E n}(t)\right]
$$

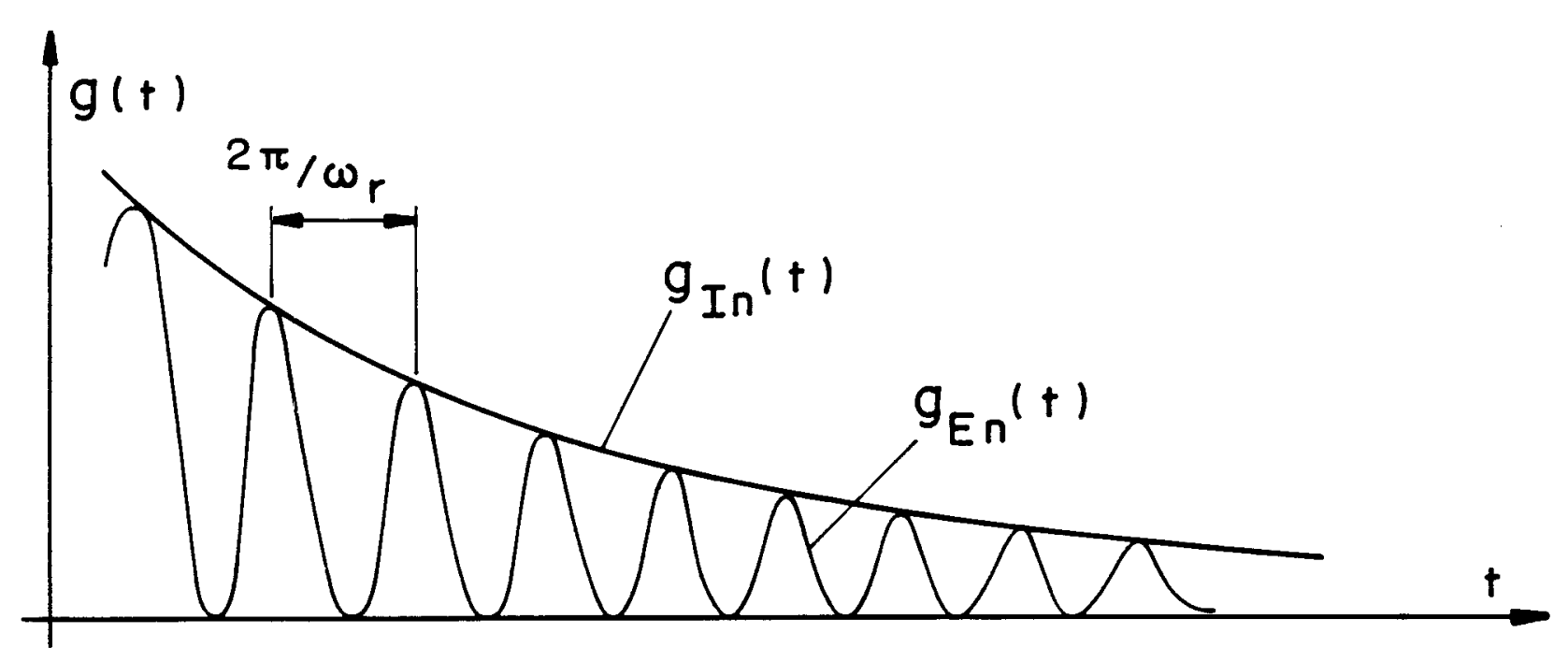

Figura 2.5: Trem de Ecos Rotacionais.

Note que temos para o FID, figura (2.5), uma parte $g_{I n}(t)$ livre de anisotropias do deslocamento químico e a outra parte $\left[\Im \otimes g_{E n}\right]$ responsável pelas anisotropias.

No espaço de freqüência, figura (2.6), resulta da transformada de Fourier em $g(t)$ :

$$
g(\omega)=\omega_{\tau} \sum_{n} g_{I n}(\omega) \otimes\left[\Im\left(\omega-\omega_{r}\right) \times g_{E n}(\omega)\right]
$$


onde utilizamo-nos dos Teoremas de Convolução ${ }^{3}$ e do fato de:

$$
\Im\left(t-\frac{2 \pi}{\omega_{r}}\right) \diamond \Im\left(\omega-\omega_{r}\right) \text {. }
$$

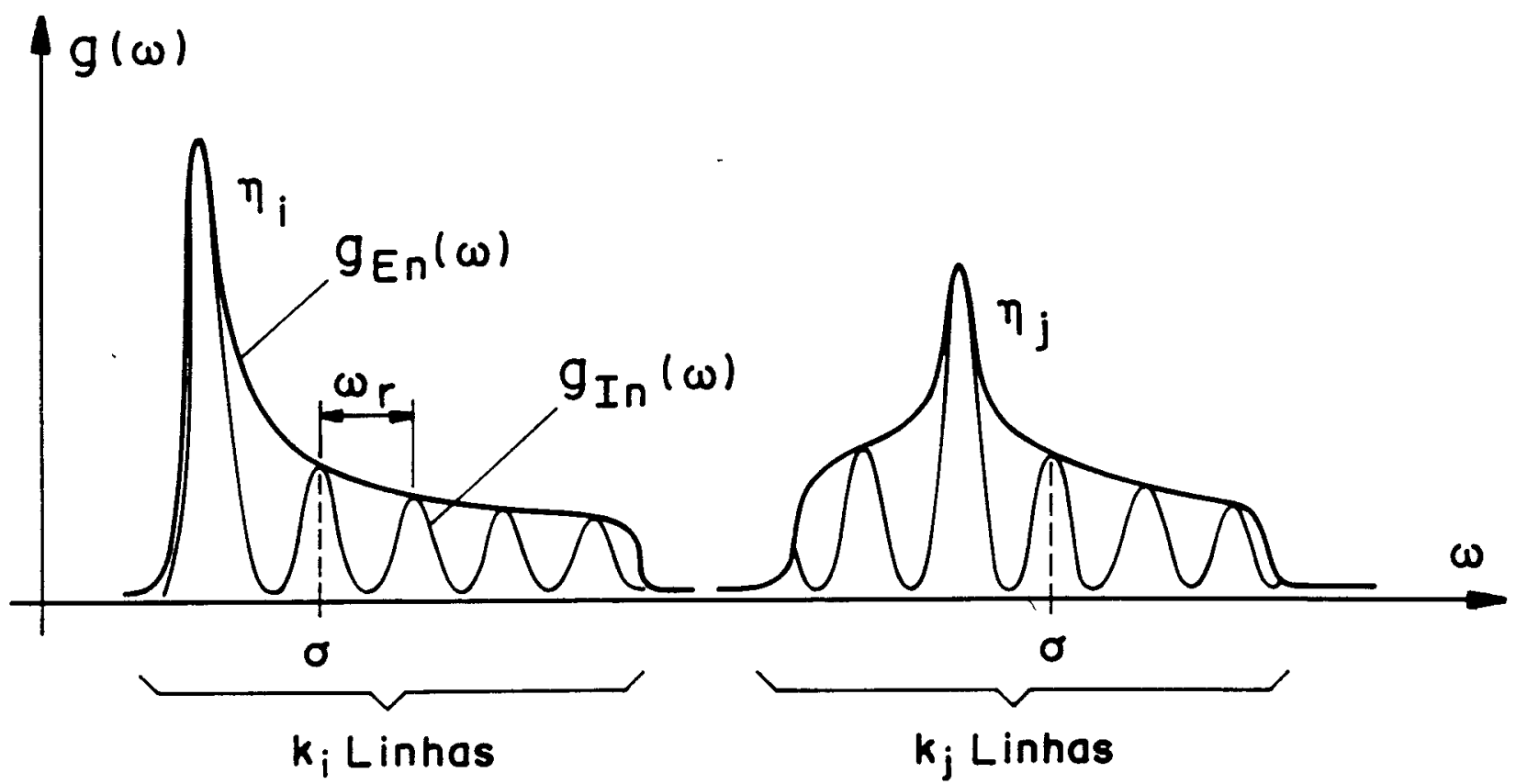

Figura 2.6: Bandas Laterais.

Observe que os fatores entre colchetes representam a transformada de Fourier de um eco rotacional amostrado num intervalo de $\omega_{r}$, isto é, uma sequência de bandas laterais, sendo que cada sequência é uma série de réplicas da n-ésima linha $g_{I n}(\omega)$ no espectro isotrópico.

Teorema 2.3.1 (Teorema da Convolução) Se $h(t)$ e $x(t)$ possuem transformadas de Fourier $H(\omega)$ e $X(\omega)$ respectivamente, então $h(t) \otimes x(t)$ possuem transformada de Fourier $H(\omega) X(\omega)$, resultando para o par de Transformada de Fourier $(\diamond)$ :

$$
h(t) \otimes x(t) \diamond H(\omega) X(\omega) .
$$

Teorema 2.3.2 (Teorema da Convolução da freqüência) Se a transformada de Fourier do produto $h(t) \times x(t)$ é igual à convolução $H(\omega) \otimes X(\omega)$, temos para o par de Transformadas:

$$
h(t) x(t) \diamond H(\omega) \otimes X(\omega) .
$$


52 CAPítULO 2. ROTAÇÃO DA AMOSTRA EM TORNO DO ÂNGULO MÁGICO 


\section{Bibliografia}

[1] E. R. Andrew, A. Bradbury and R. G. Eades, Nature 182, 1659, (195\&) e 183, 1802, (1959).

2Y I. J. Lowe, Phys. Rev. Lett. 2, 285 (1959).

[3] P. Mansfield, D. Ware, Phys. Rev. Lett., 22, 133-135, (1966).

. [4] E. D. Ostroff, J. S. Waugh, Phys. Rev. Lett., 16, 1097-1098, (1966).

c. [5] E. R. Andrew, W. S. Hinshaw, A. Jasinski, Chem. Phys. Lett. 24, 399-401 (1974).

[6] E. R. Andrew, W. S. Hinshaw, M. G. Hutchins and A. Jasinski, Proc. R. Soc. Lond., A364, 553-567, (1978).

[7] M. Matti Maricq, J. S. Waugh, J. Chem. Phys. 70, 3300-3316, (1979).

[8] J. Herfeld, A. E. Berger, J. Chem. Phys. 73, 6021-6030, (1980).

, [9] E. Lippmaa, M. Alla, T. Tuherm, in Magnetic Resonance and Related Phenomena: Proceedings of the XIX Congress Ampere, pp. 113-118, Heidelberg, September 1976.

[10] J. S. Waugh, M. M. Maricq, R. Cantor J. Magn. Res. 29, 183-190, (1978).

[11] R. Hentschel, J. Schlitter, H. Sillescu, H. S. Spiess, J. Chem. Phys. 68, 56-66, (1978).

[12] R. G. Griffin, in International School of Physics "Enrico Fermi", Societá Italiana di Fisica, Varenna (1986) - 2nd Course.

[13] E. R. Andrew, in Philosophical Transactions of The Royal Society of London - Mathematical and Physical Sciences - Nuclear Magnetic Resonance Spectroscopy in Solids, 299, 505-520, No 1452, (1981).

[14] M. Mehring, Principles of High Resolution NMR in Solids (1983), Spring Verlag.

[15] U. Haeberlen, High Resolutions NMR in Solids (1976), Academic Press.

[16] N. Chandrakumar, S. Subramanian, Modern Techniques in High Resolution FT-NMR (1987), Spring-Verlag. 
[17] V. M. S. Gil, C. F. Geraldes, Ressonância Magnética Nuclear, F. Calouste Gulbenkian, (1987).

[18] D. Shaw, Fourier Transform NMR Spectroscopy, 2nd Edition, Elsevier, (1984).

[19] E. O. Brigham, The Fast Fourier Transform, Pretice-Hall (1974). 


\section{Capítulo 3}

\section{Instrumentacão Básica}

\subsection{Introdução}

As técnicas para obtenção de informações sobre as propriedades microscópicas da matéria são vastas. A técnica de Ressonância Magnética Nuclear (RMN) de alta resolução baseada na transformada de Fourier (TF) vem desde o ano $66^{1}$, ocupando um papel importante dentre essas técnicas. Esse método espectroscópico permite-nos obter medidas de pequenas diferenças de energia que refletem em pequenas diferenças na estrutura molecular da amostra.

Separações da ordem de $0.1 \mathrm{~Hz}$ podem ser medidas, correspondendo a diferenças de energia da ordem de $10^{-13} \mathrm{Joule} / \mathrm{Mol}$. Além dessas informaçōes detalhadas da estrutura molecular podemos obter também informações a respeito de tempo de relaxação spin-rede que resulta em informaçōes da cinética e movimento molecular.

A espectroscopia por RMN é constituída basicamente por:

- Magneto, para estabelecer uma condição inicial à nossa amostra, que é um campo magnético $\mathbf{B}_{0}$, o mais estável e homogêneo possível.

- Fonte de radiofrequência para exitação do sistema de spins.

- Desacoplador.

- Sistema de detecção e registro para que possamos adquirir e processar os resultados obtidos.

O diagrama de blocos de nosso espectrômetro está esquematizado na figura 3.1.

\footnotetext{
${ }^{1}$ Surgimento dos espectrometros comerciais, Ernst \& Anderson, [2].
} 


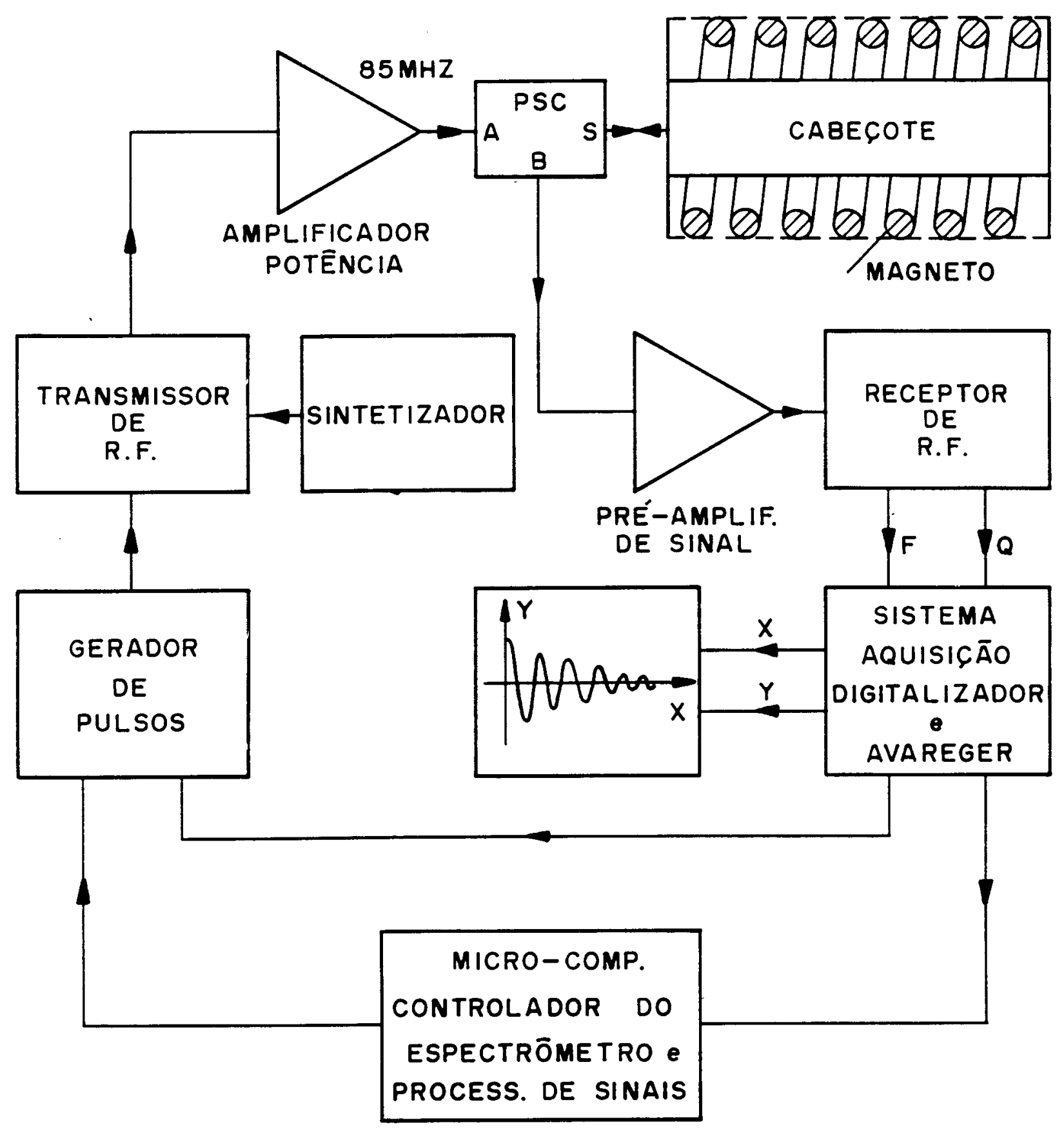

Figura 3.1: Diagrama de bloco do espectrômetro. 


\subsection{O Magneto}

O magneto uo nosso espectrômetro é um magneto supercondutor solenoidal (OXFORD $85 / 310 H R$ ), que possui as seguintes especifições:

- Campo magnético de $2.0 \mathrm{~T}(85 \mathrm{MHz})$.

- Estabilidade de campo de $0.1 \mathrm{ppm} /$ hora.

- homogeneidade de campo até $0.25 \mathrm{ppm}$, isso para uma esfera de diâmetro de $20 \mathrm{~mm}$.

- Sete bobinas de homogeneização de campo supercondutoras, responsáveis pelas ordens de correção:

$$
\mathrm{X}, \mathrm{Y}, \mathrm{Z}, \mathrm{ZX}, \mathrm{ZY}, \mathrm{XY}, \mathrm{e}\left(\mathrm{X}^{2}-Y^{2}\right) \text {. }
$$

- Quinze bobinas de homogeneização de campo à temperatura ambiente, responsáveis pelas ordens de correção:

$$
\begin{aligned}
& Z_{0}, Z_{1}, Z_{2}, Z_{3}, Z_{4} \mathrm{X}, \mathrm{Y}, \\
& \mathrm{ZX}, \mathrm{ZY}, \mathrm{XY}, \mathrm{ZX}, \mathrm{ZY}, \\
& \mathrm{ZXY}, Z\left(X^{2}-Y^{2}\right),\left(X^{2}-Y_{2}\right) .
\end{aligned}
$$

- Diâmetro útil de $310 \mathrm{~mm}$.

- Comprimento útil de $947 \mathrm{~mm}$.

\subsection{Fonte de Radiação}

Utilizamos como fonte de rádio-freqüência um cristal de alta estabilidade, que é parte principal dum sintetizador de RF (WAVETEK - 5120A), que fornece sinal para modulação e referência na deteç̧ão. Este aparelho é capaz de gerar sinais de $500 \mathrm{~Hz}$ a $160 \mathrm{MHz}$, ajustáveis em passos de $0.001 \mathrm{~Hz}$.

Os sinais provenientes deste sintetizador entram no transmissor o qual é capaz de prover pulsos de RF retangulares de curta duração (microsegundos) préviamente programados, controlados pelo gerador de eventos. Tal transmissor também fornece sinais contínuos em fase e quadratura $\left(0^{\circ}, 90^{\circ}\right)$, utilizados como referência no receptor de sinais de RMN.

Os pulsos vindos do transmissor são amplificados por um amplificador de RF ( $A M$ PLIFIER RESEARCH - 200L). Esses, depois de amplificados, passam por um duplexador que resulta numa isolação entre transmissor de RF e o receptor de sinais de RMN. Tal duplexador tem por função dirigir ao ressoador os pulsos de potência de RF, produzidos pelo transmissor (capaz de gerar potência pulsada de $700 \mathrm{~W}$ ), na fase de excitação e por sua vez dirigir os sinais pela amostra no ressoador ao receptor de sinais na fase de recepçāo. 
Esses sinais de RMN gerados pela amostra são amplificados depois de passarem pelo duplexador tornando-se adequados à demodulação no receptor.

\subsection{Recepção do Sinal}

O receptor dos sinais gerados pela amostra é dividido em três fases distintas: amplificação de $R F$, demodulação, fltragèm de áudio (passa-baixas até $300 \mathrm{KHz}$ ), essas duas últimas, duais. O sinal demodulado é dirigido para o sistema de digitalização, promediação e visualização num monitor XY. Posteriormente o sinal pode ser enviado a um microcomputador para ser processado.

\subsection{Sistema de Controle}

Utilizamos para controlar toda essa dinâmica de aquisição e radiação um gerador de eventos, que foi desenvolvido em nosso labotório [1]. Esse por sua vez gera os diferentes intervalos de tempos necessários nas sequências de pulsos que controlam o sincronismo do experimento. Observe que o gerador de eventos controla praticamente todos os componentes de um espectrômetro.

Esse gerador é capaz de produzir pulsos com estreita relação de sincronismo entre si em 8 canais independentes. $O$ número de eventos em uma sequência pode chegar a 16 , podendo qualquer parte da sequência ser repetida até 999 vezes.

Sua programação é feita através do microcomputador por meio de uma linha serial.

\subsection{Cabeçote de RMN}

O cabeçote que utilizamos para espectroscopia de alta resolução em sólidos possui duas partes distintas: sonda de RF e Rotores para rotação da amostra em torno do ângulo mágico

Discutiremos neste capítulo, a sonda de RF, ficando a parte da rotação da amostra, que diz respeito à técnica que implementamos, num capítulo à parte.

A sonda de RF que utilizamos em nosso cabeçote é constituída de uma bobina solenoidal, contendo no seu interior, a amostra para excitação dos núcleos atômicos e posteriormente captar o sinal de RF proveniente da amostra. Aplicamos sobre essa bobina uma corrente alternada com freqüência idêntica a de Larmor e intensidade suficiente o bastante para tirarmos a magnetização da direção ao longo do campo estático, isto é de sua posição de equilíbrio.

Além dessa bobina, o circuito do nosso cabeçote é constituído de capacitores, resultando num circuito ressonante; assim podemos intensificar o valor do campo de RF produzido pela bobina. Sabendo-se que a impedância padrão dos equipamentos de RF é de $50 \Omega$, procuramos estabelecer através do circuito que montamos um casamento de 
impedâncias pois a amostra (carga) que queremos excitar está dentro de um solenóide, isto é, uma indutância.

Tal circuito é representado pela figu: a 3.2, onde vemos um capacitor em série $C_{\boldsymbol{s}}$ e um em paralelo $C_{p}$ com a bobina $\mathrm{L}$. Observe que podemos com a variação de $C_{s}$ controlar a impedância e com $C_{p}$ controlar a sintonia do circuito. Tais capacitâncias são calculadas por:

$$
\begin{aligned}
& C_{s}=\left[\left(\left(Q \omega L-R_{0}\right) R_{0} \omega^{2}\right]^{-\frac{1}{2}}\right. \\
& C_{p}=\frac{Q R_{0} \omega C_{s}-1}{Q R_{0} \omega^{3} C_{s} L}
\end{aligned}
$$

onde a indutância $\mathrm{L}$ e seu fator de qualidade $\mathrm{Q}^{2}$, são determinados experimentalmente por um medidor de fator de qualidade (HEWLETT-PACKARD - 260A), e por sua vez $R_{0}=50 \Omega$ que é a impedância padrão do equipamento e $\omega$, a freqüência de ressonância do circuito, que deve ser igual à freqüência de ressonància dos núcleos - Larmor $\omega=\gamma B_{0}$.

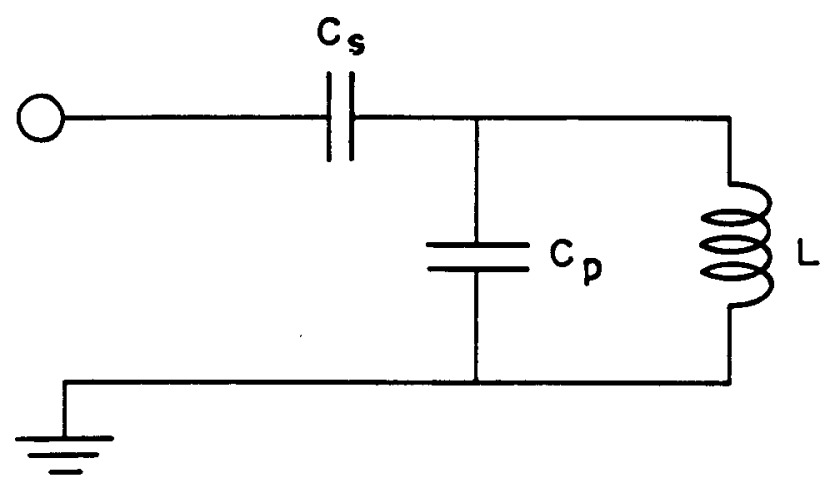

Figura 3.2: Circuito de RF, para exitação dos núcleos atômicos e deteç̧ão de sinal.

Nesse nosso cabeçote utilizamos a mesma bobina de excitação como bobina de deteç̧ão de sinal.

\subsection{Cálculos para a Sonda de RF}

Quando nos propomos a construir cabecotes de RMN, temos que levar em conta vários fatores. Um deles é quanto à dimensão da amostra que será alojada no interior da bobina. Isso vem do fato que o campo de $\mathrm{RF}, \mathbf{B}_{1}$, produzido pela bobina é dado por Clarck [9] como:

\footnotetext{
${ }^{2} Q=\omega \frac{L}{R}$, onde $\mathrm{L}$ e $\mathrm{R}$ são a indutância e a resistência da bobina.
} 


$$
\mathbf{B}_{1} \cong 3\left(\frac{P Q}{V \mu}\right)^{\frac{1}{2}}
$$

onde $\mathrm{P}$ é a potência de RF transmitida em watts ao circuito, $\mathrm{Q}$ é o fator de qualidade do indutor, $\mu$ é a freqüência de Larmor em $\mathrm{MHz}$ e V o volume da bobina em $\mathrm{cm}^{3}$.

Observe que $Q$ pode ser feito grande para minimizar a resistência e maximizar a indutância para uma dada freqüencia de ressonância $\omega$. Isto maximiza $\mathbf{B}_{1}$ durante o pulso e incrementa a sensibilidade do sinal de RMN.

$\mathrm{O}$ volume da amostra $\mathrm{V}$ alojada na bobina deverá ser pequeno para maximizar $\mathbf{B}_{1}$. Porém a relação sinal/ruído $(S / R)$ é proporcional ao número de núcleos da amostra e ao quadrado do fator de preenchimento. Disso resulta a necessidade de uma dimensão adequada para a bobina de acordo com a quantidade média da amostra que se utiliza.

Além desse fator geométrico, precisamos levar em conta que a freqüencia de auto ressonância ${ }^{3}$ da bobina nāo pode ser próxima a freqüência de trabalho de $85 \mathrm{MHz}$, e por sua vez a bobina tem que se adaptar ao nosso sistema de rotação. Estes fatores levam-nos a trabalhar com fio de cobre fino e com uma bobina com poucas voltas.

Para se obter uma bobina com Q grande e boa homogeneidade de campo utilizamo-nos de parâmetros técnicos estabelecidos pela experiência em construção de bobinas:

- Utilizamo-nos de bobinas solenoidais com o comprimento correspondendo a cerca de $70 \%$ do seu diâmetro [4], figura 3.3 .

- A distância entre duas espiras deve ser no mínimo igual ao triplo do raio dos condutores [7\}, figura 3.3.

Para calcular o número de voltas necessárias para uma determinada indutância $\mathrm{L}$, temos de Landee [8]:

$$
L=\frac{4 n^{2} a^{2}}{7 a+8 g}
$$

onde a e g estão representados na figura 3.3. Observe que (3.4) é uma fórmula que ajuda a estimar o número de voltas, porém o número exato tem que ser obtida empiricamente.

$\mathrm{O}$ valores de $\mathrm{Q}, C_{L}$ e $\omega_{T}$ (freqüência de trabalho) são obtidos pelo $Q$-meter, donde obtemos os vários valores de $Q$ em função de $\omega_{T}$, podendo-se estimar o valor de $Q$ para $f_{0}$ e por sua vez, obtemos o valor de L da condição de sintonia:

$$
L C_{L} \omega_{T}^{2}=1
$$

Utilizando-se de (3.1) e (3.2) obtemos os valores de $C_{p}$ e $C_{s}$ conforme está apresentada na tabela 3.1. Para obtermos esses resultados utilizamo-nos dos valores de $Q, L$ e do fato de que nosso espectrômetro possui um campo de 2 tesla, resultando numa freqüência de ressonância para $H^{1}$ de aproximadamente $85.24 \mathrm{MHz}$.

\footnotetext{
${ }^{3}$ freqüência de Auto Ressonância: freqüência na qual a bobina ressoa com sua própria capacitância distribuida.
} 


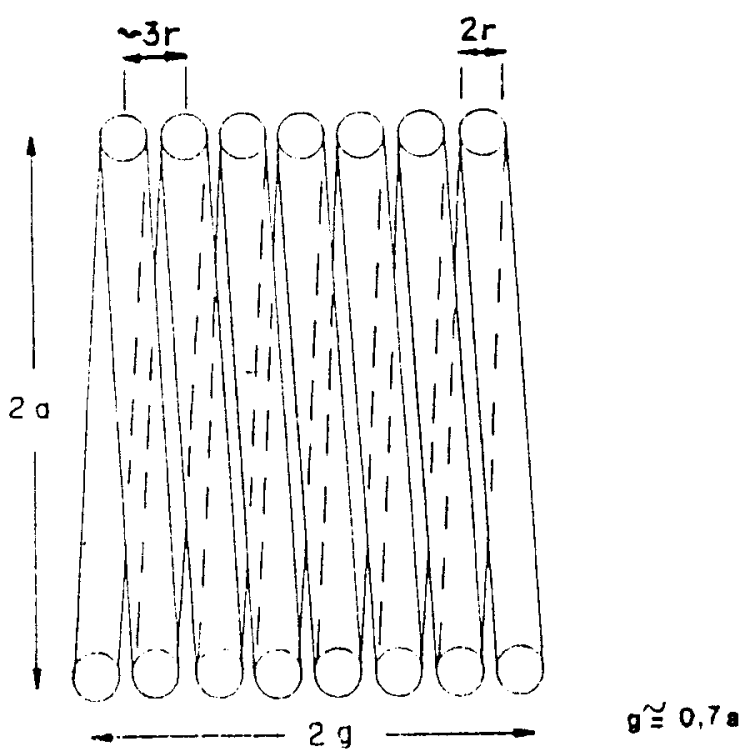

Figura 3.3: Geometria "Otima" para bobinas solenoidais.

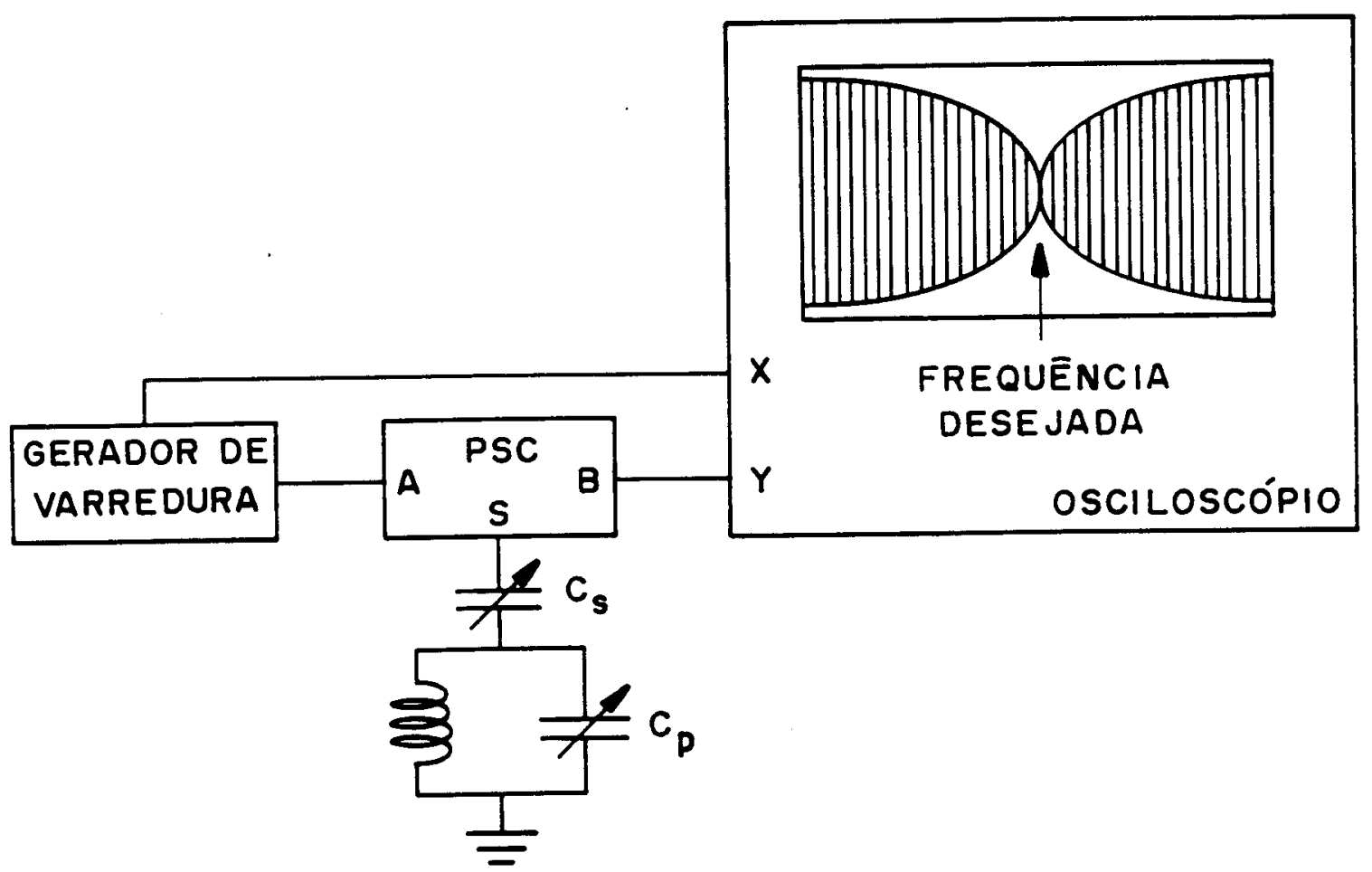

Figura 3.4: Condição de Sintonização. 


\begin{tabular}{|c|c|c|c|c|c|}
\hline Bobina & $\mathrm{Q}$ & $\mathrm{L}[\nu h]$ & $C_{s}[p F]$ & $C_{p}[p F]$ & Capacitor Utilizado \\
\hline 1 & 90.0 & 0.7 & 1.5 & 3.3 & $0.8 \mathrm{pF} \leftrightarrow 10 \mathrm{pF}$ \\
2 & 95.5 & 0.8 & 1.3 & 2.5 & $0.8 \mathrm{pF} \leftrightarrow 10 \mathrm{pF}$ \\
\hline
\end{tabular}

Tabela 3.1: Valores de $C_{s}$ e $C_{p}$ obtidos para nosso cabeçote.

Um outro fator que deve ser levado em conta são os ruídos produzidos pela sonda. A microfonia é um dos ruídos que devem ser evitados, isto é vibraçōes mecânicas da sonda que afetam o sinal. Por isso é necessária uma boa fixação da bobina de RF e de outros componentes, mesmo porque a nossa amostra estará girando, fato que é propício a provocar vibraçōes e também durante o pulso, uma corrente flui na bobina, essa por sua vez imersa no campo magnético externo $\mathbf{B}_{0}$, resultando em grandes forças sobre a bobina causando vibraçōes mecânicas, resultando em ruídos que se evidenciam após aplicação dos pulsos.

Tais vibraçōes podem modular a capacitância existente entre a bobina e a própria estrutura da sonda, resultando numa sintonia degenerada. Ainda devemos estar seguros de que as soldas estão bem feitas. Soldas mal feitas resultam numa degradação do sinal de RMN.

Os equipamentos que fazem parte da sonda de RF, também devem ser protegidos pois no cabeçote de RMN manipulamos potências de RF que podem variar de dezenas à centenas de watts. Para evitar faiscamentos e deteriorização dos pulsos de RF os circuitos de RF devem ser cuidadosamente confeccionados.

Um outro fato ainda que deve ser levado em conta, é a não utilização de materiais que possuam o núcleo que estamos interessados em excitar, na construção do cabeçote e seus componentes. Em nosso cabeçote utilizamo-nos de alumínio na estrutura externa e de suporte, por ser um material não magnético, servindo de blindagem de sinais externos a sonda aterrada adequadamente. Quanto ao suporte para nossa bobina de RF, utilizamonos de TEFLON e DELRIN ${ }^{4}$. Sua construção será discutida detalhadamente no capítulo que descreve a construções de rotores para a técnica de ângulo mágico.

\subsection{Montagem da Sonda de RMN}

Nosso circuito, figura 3.2 , depois de calculado é montado sobre suas bases circulares de alumínio, sendo que o cilindro estabelecido por essas duas bases é separado em quatro partes por duas chapas de alumínio, conforme a figura 3.7 .

Com essa separação estamos aptos a instalar os circuitos de RF, em compartimentos - separados e blindados eletromagneticamente entre si, de modo a evitar o acoplamento mútuo entre cada circuito. No caso estamos interessados somente no núcleo de $H^{1}$ e utilizamo-nos somente de uma parte para instalação do circuito de RF. Porém, a estrutura da sonda fica apta a ser utilizada para futuras instalações de outros circuitos quando

\footnotetext{
${ }^{4}$ DELRIN é nome de marca registrada da E. I. Dupont DeNemours Co. para a resina de poliamida.
} 
quisermos, por exemplo, trabalhar com dupla ressonância.

Essa bases cilíndricas de suporte e blindagem do circuito de RF são adaptadas num tubo dé alumínio de $12 \mathrm{~cm}$ de diâmetro, que também serve de suporte e blindagem geral, figura 3.7. Por sua vez, esse tubo de alumínio possui anéis de acrílico que servem como suporte para sua fixação no magneto; além desses anéis, possui uma flange de alumínio em uma de suas extremidades, que é fixada ao magneto por parafusos metálicos, que atuam também como terra comum entre o cabeçote e o magneto. $\mathrm{Na}$ extremidade oposta possui uma tampa de alumínio que além de blindagem, serve de suporte para "portas laterais", que nada mais são que duas tampas semi-cilíndricas que podem ser retiradas quando for necessária a troca de amostra ou manutenção, figura 3.5; além dessa função, esta tampa de alumínio serve como sustentação de uma barra de fenolite, que é eixo de uma engrenagem diferencial que serve para ajuste do ângulo mágico e também como suport a para o bocal de alimentação de ar comprimido do rotor, figura 3.6.

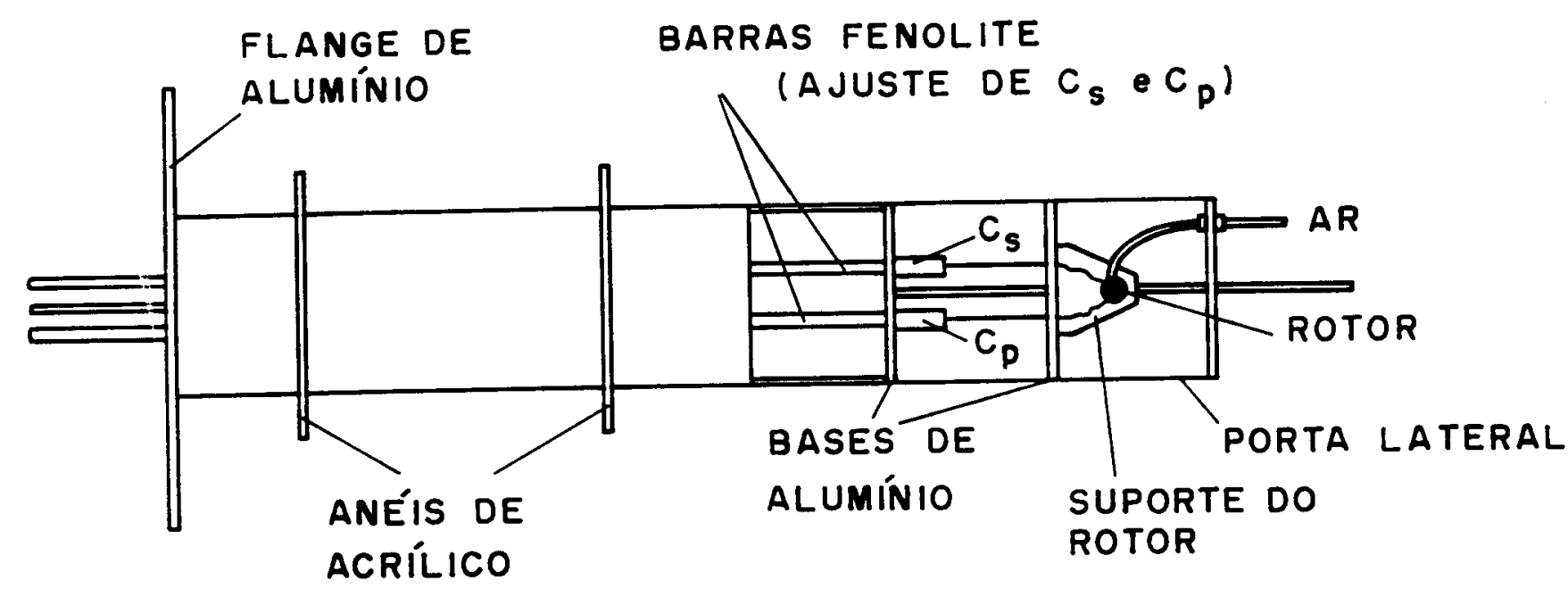

Figura 3.5: Aspecto geral do cabeçote de RMN.

Também utilizamos-nos de barras de fenolite para ajustar os capacitores variáveis, que são fixadas nos próprios capacitores e na flange de fixação de alumínio, figura 3.7. Todas as barras possuem travas de segurança para evitar que os capacitores saiam da sintonia. 


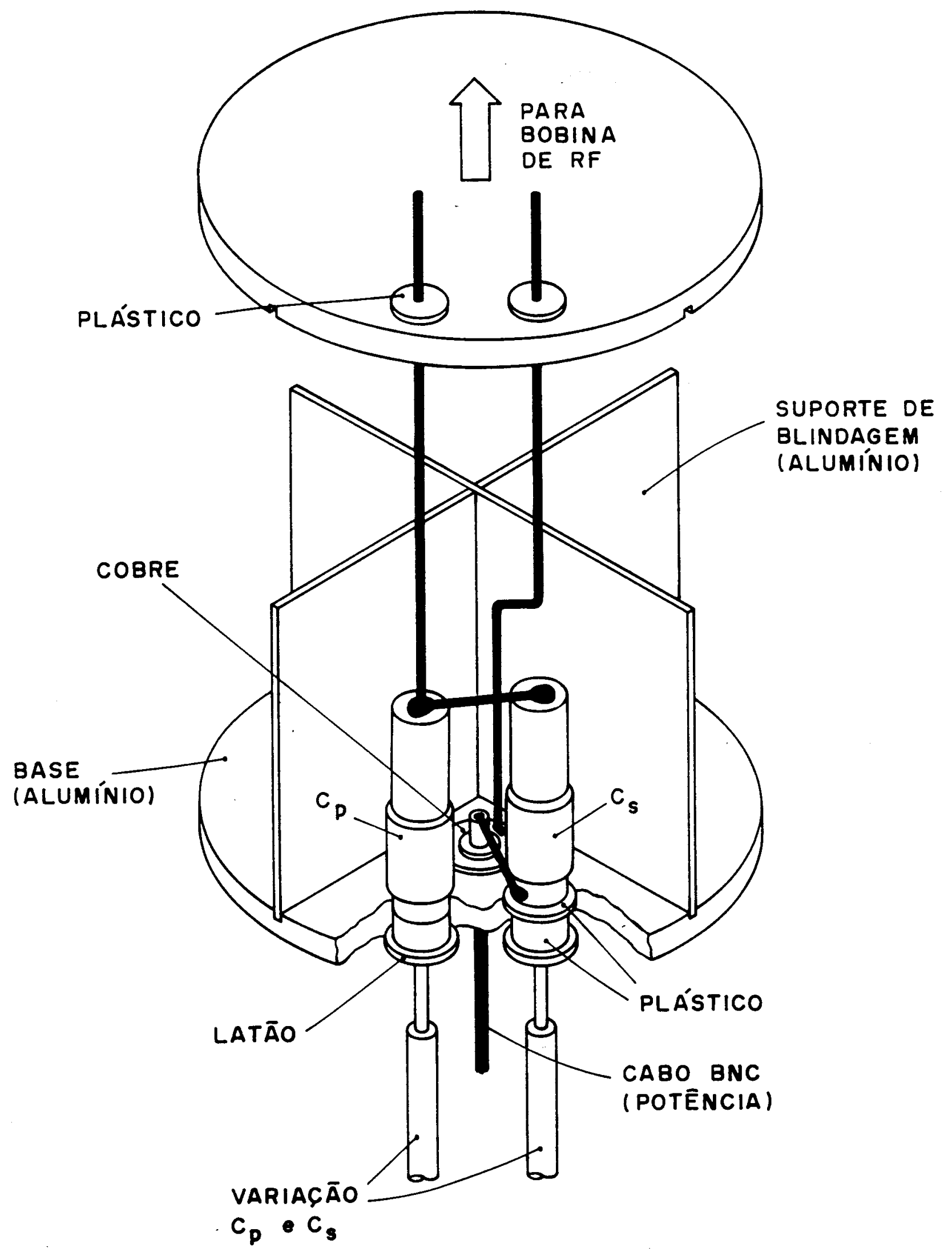

Figura 3.7: Representação dos compartimentos onde montamos nosso cireuito de $R F$. 
CAPITULO 3. INSTRUMENTACÁO BÁSICA 


\section{Bibliografia}

[1] M. S. Veludo de Paiva, Construção de um Gerador de Pulsos Programáveis para experiência de RMN Pulsada - Tese de Mestrado DFCM - IFQSC - LSP (1983).

[2] R. R. Ernst and W. A. Anderson, The Review of Scientific Instruments, 37, number $1,93-102$, January 66.

[3] D. I. Hoult, R. E. Richards, The signal-to-noise Ratio of NMR Experiment, J. Mag. Res. 24, 71-85 (1986).

[4] D. I. Hoult, P. C. Loutebur, Sensitivity of Zegmatografic Experiment Involving Human Samples, J. Mag. Ress., 34, 425-433, (1970).

[5] Manual da Bruker - "High Resolution NMR of Solids".

[6] T. J. Bonagamba, Relatório de período probatório 88/90, Projeto: Espectroscopia de Alta Resolução em Sólidos por RMN.

[7] F. E. Terman, Eletronic and Radio Enginneering.

[8] R. W. Landee, D. C. Davis and A. P. Albrecht, The Electronic Designers' Handbook, MacGraw Hill Book Co., N.York, 1957.

[9] W. G. Clarck, Pulsed Nuclear Resonance Apparatus, Rev. Sci. Instrum. 35, 316-333 (1964).

[10] E. R. Andrew, W. S. Hinshaw, M. G. Hutchins and A. Jasinski, Proc. R. Soc. Lond., A364, 553-567, (1978).

[11] M. Matti Maricq, J. S. Waugh, J. Chem. Phys. 70, 3300-3316, (1979).

[12] J. Herfeld, A. E. Berger, J. Chem. Phys. 73, 6021-6030, (1980).

[13] E. Lippmaa, M. Alla, T. Tuherm, in Magnetic Resonance and Related Phenomena: Proceedings of the XIX Congress Ampere, pp. 113-118, Heidelberg, September 1976.

[14] J. S. Waugh, M. M. Maricq, R. Cantor J. Magn. Res. 29, 183-190, (1978).

[15] R. Hentschel, J. Schlitter, H. Sillescu, H. S. Spiess, J. Chem. Phys. 68, 56-66, (1978).

67 
[16] R. G. Griffin, in International School of Physics "Enrico Fermi", Societá Italiana di Fisica, Varenna (1986) - 2nd Course.

[17] E. R. Andrew, in Philosophical Transactions of The Royal Society of London - Mathematical and Physical Sciences - Nuclear Magnetic Resonance Spectroscopy in Solids, 299, 505-520, No 1452, (1981).

[18] M. Mehring, Principles of High Resolution NMR in Solids (1983), Spring Verlag.

[19] U. Haeberlen, High Resolutions NMR in Solids (1976), Academic Press.

[20] N. Chandrakumar, S. Subramanian, Modern Techniques in High Resolution FT-NMR (1987), Spring-Veriag.

[21] V. M. S. Gil, C. F. Geraldes, Ressonância Magnética Nuclear, F. Calouste Gulbenkian, (1987).

[22] D. Shaw, Fourier Transform NMR Spectroscopy, 2nd Edition, Elsevier, (1984).

[23] E. O. Brigham, The Fast Fourier Transform, Pratice-Hall (1974). 


\section{Capítulo 4}

\section{Sistema de Rotação da Amostra}

\subsection{Introdução}

Quando nos propomos a construir um sistema de rotação para girar nossa amostra para espectroscopia em sólidos por RMN, levamos em conta a necessidade de rotores com operação em alta rotação e alta estabilidade. Tais necessidades leva-nos a utilizarmos rotores lubrificados a ar, isto é Mancais Aerostáticos, pelo seu excelente desempenho devido, ao seu baixo nível de ruído e atrito praticamente nulo.

Esses mancais aerostáticos podem ainda ser utilizados com grande variação de temperatura tornando-se eficientes para experimentos que necessitam de tais variações. Porém, faz-se necessário levar em conta que para tais projetos com mancais aerostáticos, requerse um projeto cuidadoso para evitar-se vibrações devido à compressibilidade do ar; isso impõe extrema precisão e estabilidade de dimensōes limitando-nos na escolha de materiais.

A utilização de mancais lubrificados a óleo não é satisfatório pois um grande fluxo de óleo de baixa viscosidade seria requerida para evitar um calor execessivo na superfície do mancal; além do mais, um sistema de recuperação do óleo é incoveniente levando-se em conta os vínculos de um experimento de RMN.

Historicamente tais rotores utilizados em espectroscopia por RMN, podem ser divididos em dois tipos: os de Andrew [2] - que emprega uma superfície de ar do mancal e da turbina cônicos - e os posteriores que utilizam-se de sistemas cilíndricos com sistema de mancal e propulsão separados. Os sistemas de rotação de Andrew são fáceis de serem fabricados, porém, não é possível de se otimizar conjuntamente a eficiência de propulsão e a estabilidade de tais rotores, principalmente com amostras inhomogêneas em alta rotação.

Por isso, em nosso projeto, utilizamo-nos de rotores com sistema de propulsão e mancal separados, conforme Doty 6 Ellis [1], que possuem duas partes fundamentais: o Mancal de Sustentação e Lubrificação do Rotor e Sistema de Propulsão do Rotor, que iremos discutir separadamente nas próximas seções. 


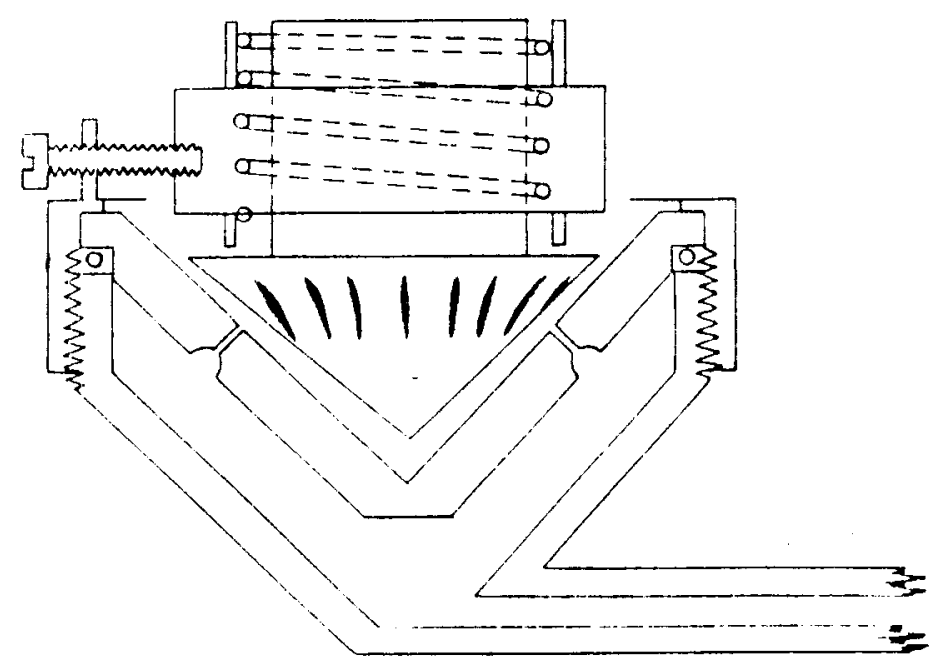

Figura 4.1: Sistema de rotação da amostra de Andrew et al (1969), fonte: [3].

\subsection{Mancais Aerostáticos Radiais}

A característica principal de um mancal é a rigidez da película de fluído que escoa entre as duas superfícies que determinam o mancal, figura (4.2). Essas películas são mantidas numa determinada pressão de sustentação devido a uma fonte de alimentação externa com pressão constante.

Observe que o furo de diâmetro d (restritor) possui um papel fundamental, que é controlar a vazão de ar entre a fonte de alimentação e a folga (h) do mancal, controlando assim sua rigidez. Segundo Powell [4] a rigidez $(K)[N / m]$ dos mancais é dado por:

$$
K \propto \frac{W_{c}}{h}
$$

sendo $W_{c}$ a carga, que é fixa, necessitamos para aumentar a rigidez de nosso mancal, diminuir a folga $(\mathrm{h})$. Observe que a carga $W$ que o mancal deve suportar devido à gravidade e impulsos das aletas propulsoras é insignificante perto da carga radial $W_{\text {r }}$ produzida devido ao empacotamento assimétrico da amostra. Considerando-se uma amostra com uma quantidade de massa desbalanceada $\left(m_{a}\right)$ que encontra-se num raio $r$ e com uma velocidade angular $\omega$, resulta da segunda lei de Newton para $W_{r}$ :

$$
W_{r}=m_{a} \omega^{2} r
$$

Para uma amostra em pó bem empacotada, $m_{a}$ é em torno de $1 \%$ da massa total da amostra, enquanto para um amostra que seja um polímero granular temos que $m_{a}$ é em torno de $10 \%$ da massa total da amostra. Um exemplo, uma amostra de um grama $\left(m_{a}=0.01 \mathrm{~g}\right)$ num raio médio de $4 \mathrm{~mm}$ e com uma velocidade angular de $3.2 \mathrm{kHz}$, resulta numa carga de $16.7 \mathrm{~N} !\left(1.6 \mathrm{~kg}\right.$ para $\left.g=10 \mathrm{~m} / \mathrm{s}^{2}\right)$, observe que $W_{r} \gg W_{c}$. 


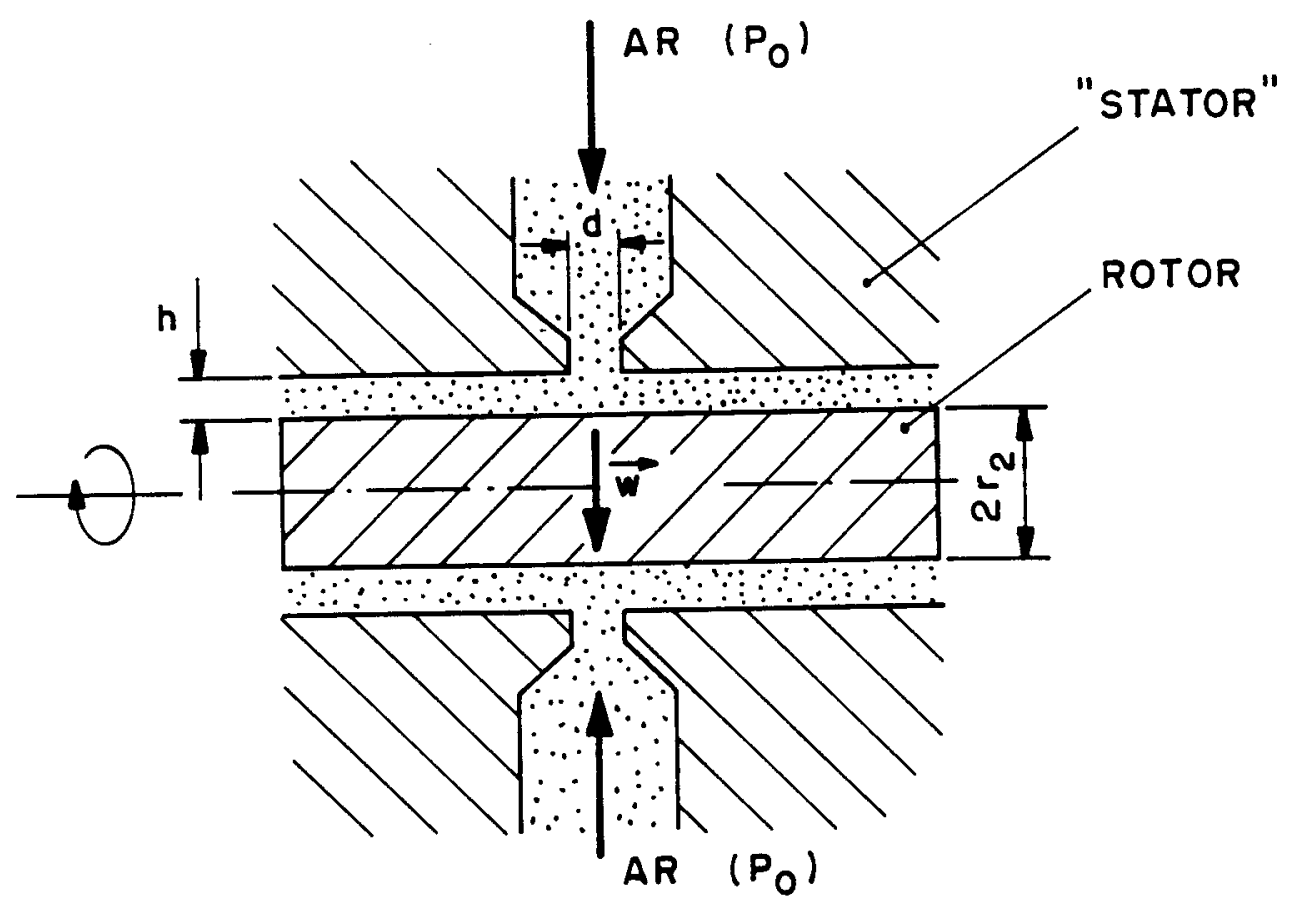

Figura 4.2: Mancal aerostático.

Precisamos entāo dimensionar nosso mancal e por sua vez sua folga (h), levando-se em conta que tais mancais precisam ter uma capacidade pelo menos igual à carga radial $W_{r}$. Segundo Doty \& Ellis [1], uma folga radial ótima (h) é dada por:

$$
h \simeq\left[\frac{7000 \mu^{2} r_{2}}{c^{2} \rho^{2}}\right]^{\frac{1}{3}}
$$

onde $\mu$ é a viscosidade dinâmica do gás, $\mu_{a r}=1.8 \times 10^{-4} d y n s / \mathrm{cm}^{2}$, c é a velocidade do som ${ }^{1}, \rho$ é a densidade do gás, $\rho_{a r}=0.0754 \mathrm{lb} / \mathrm{ft}$ a $20^{\circ} \mathrm{C}$ e $r_{2}$ é o raio do rotor, figura (4.2). Esta quantidade é aproximadamente $2.0 \times 10^{-3} \mathrm{~mm}$ para um rotor de $6.0 \mathrm{~mm}$ de diâmetro com ar numa pressão de $\approx 3.0 \mathrm{~atm}^{2}$. Apesar dessa aproximação para a folga $(\mathrm{h})$, é aconselhável, sempre que possível, trabalhar com a menor folga possível, assim teremos um aumento da rigidez dos mancais.

Um outro fator experimental que devemos levar em conta é o fato da velocidade máxima praticável do rotor ser aproximadamente $0.5 c$, resultando uma freqüência máxima

\footnotetext{
${ }^{1}$ Velocidade do som:
}

$$
c=\left[\frac{\gamma p}{\rho}\right]^{\frac{1}{2}}
$$

onde $p$ é a pressão, $\gamma$ a razão de calor específico do gás, $\gamma=1.41$ para o gás diatômico.

${ }^{2}$ Essas dimensões sāo do nosso projeto. 
$\left(f_{\text {max }}\right)$ dada por:

$$
f_{\max } \simeq 0.08 \frac{c}{r_{2}} .
$$

Para estimarmos o diâmetro do furo de alimentação do mancal $(d)$, assumimos uma pressão de trabalho de $3 \mathrm{~atm}$, um comprimento do furo desprezível e também desprezamos os efeitos inerciais em torno do furo, resultando:

$$
d \sim\left[2.8 \frac{h^{3}}{c}\right]^{\frac{1}{2}} \times 10^{4}
$$

Para o nosso rotor de diâmetro de $6 \mathrm{~mm}$ isso resulta $d \sim 0.01 \mathrm{~mm}$. Nessa aproximação ignoramos as perdas devido a viscosidade e turbulência no e em volta do furo de alimentação e assumimos também um fluxo irrotacional. Levando-se em contz. essas perdas sugere-se o aumento de $10-30 \%$ no raio desse furo.Uma maneira de auxiliar o escoamento de ar nesses furos de alimentação é através de "rebaixos" construidos nas entradas desses furos.

\subsection{Turbina}

No projeto de rotores outro tópico que deve ser levado em conta é o sistema de propulsão dos rotores. Uma maneira ilustrativa do princípio de propulsão do rotor é mostrada na figura (4.3), onde a propulsão é realizada pelo jato de ar em alta velocidade que incide sobre as aletas. Esses jatos de ar são alimentados por uma fonte externa de ar comprimido em alta pressão.

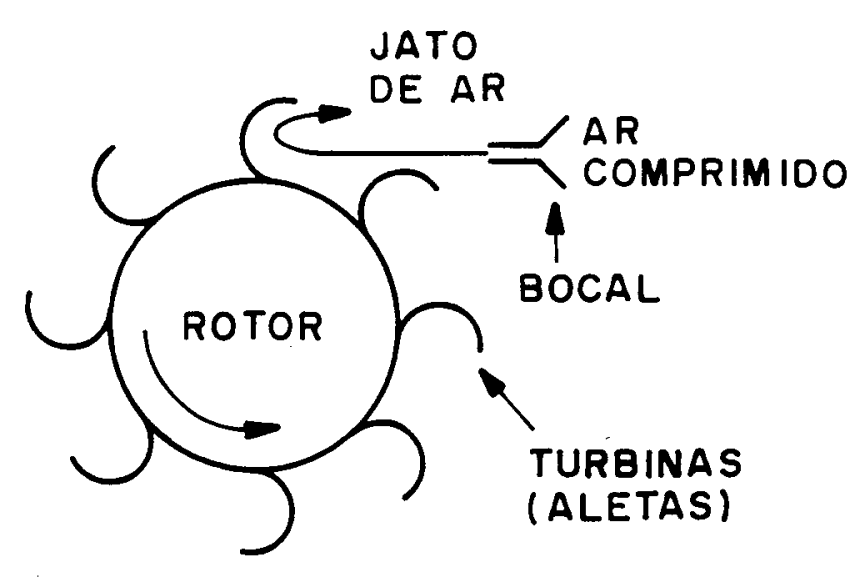

Figura 4.3: Princípio de propulsão do rotor.

Sendo assim faz-se necessário o dimensionamento do sistema de propulsão para melhor eficiência de rotação. Isso foi feito por Doty \& Ellis [1], a partir da experiência adquirida em vários projetos de rotores. Um ponto importante do projeto do sistema de propulsão são os orifícios de alimentação do jato propulsor. Esses deviam possuir um formato 
afunilado evitando-se estrangulamentos na passagem do ar, isto é, tornando o seu caminho o mais suave possível.

Porém, tais afunilamentos sāo muito difíceis de serem feitos na prática, requerendo alta sofistificação de maquinário. Assim, o que procuramos fazer é um furo de alimentação o mais curto possível, evitando-se perdas de velocidades. Para um rotor com velocidade de $0.5 c$ e um jato com velocidade propulsora de c, o raio desse oríficio é estimado de:

$$
r_{j} \simeq 0.02\left[r_{2}^{\frac{2}{3}} \ell\right]^{\frac{1}{2}}
$$

Infelizmente, a experiência mostra-nos que essa estimativa é metade do valor real. Isto sugere outros mecanismos de perda que nāo levamos em conta tal como vibração do rotor e especialmente turbulências no jato e nas aletas do rotor. Para o nosso projeto com um rotor de $6 \mathrm{~mm}$ de diâmetro, resulta para o raio desse oríficio, $0.03 \mathrm{~mm}$. Por sua vez uma profundiade ótima, $h_{a}$ para as aletas, é de 0.9 vezes o diâmetro do jato, resultando $h_{a}=0.54 \mathrm{~mm}$ para o nosso projeto.

\subsection{Seleção dos Materiais}

O projeto de rotores para espectroscopia em sólidos impõe várias restrições no que diz respeito à escolha de materiais para confeccioná-los. A folga para o mancal aerostático é de $\sim \frac{5}{1000}$ do diâmetro do rotor; isso requer alta precisão e para tal precisamos levar em conta as propriedades físicas do material, que para alguns materiais plásticos, cerâmicos e vítreos estão relacionados na Tabela (4.1).

Lembrando que a pressão de esforço $p_{m}$ num cilindro de raio $\mathrm{r}$ e com uma parede fina de espessura é dado por:

$$
p_{m}=T \frac{e}{r}
$$

onde $\mathrm{T}$ é a resistência a tensāo do material.

Por sua vez a tensão radial $\epsilon$, que é o aumento porcentual no raio causada pela pressão centrífuga é dada por:

$$
\epsilon=\frac{p, r}{Y e}
$$

onde $Y$ é o modulo de Young de elasticidade do material. Por sua vez, da equação (4.1) temos que a pressão centrípeta $p$, na superfície do rotor necessária, para suportar o tensor de densidade $\rho$ e raio $r$ numa velocidade angular $\omega$ é dada por:

$$
p_{s}=\frac{1}{2} \omega^{2} r^{2}
$$

Para o caso de um rotor que contenha uma amostra de densidade $\rho_{1}$, raio interno $r_{i}$, raio externo $r_{2}$ e feito de um material com densidade $\rho_{2}$, a pressão total será: 


\begin{tabular}{|c|c|c|c|c|c|c|}
\hline \multirow[b]{2}{*}{ Material } & \multirow{2}{*}{$\begin{array}{c}\text { Resist ência } \\
\text { a Tensão } \\
\text { dyn } / \mathrm{cm}^{2} \times 10^{\circ}\end{array}$} & \multirow{2}{*}{$\begin{array}{c}\text { Modulo de } \\
\text { Young } \\
\text { dyn } / \mathrm{cm}^{2} \times 10^{10} \\
\end{array}$} & \multirow{2}{*}{$\begin{array}{c}\text { Densidade } \\
\mathrm{g} / \mathrm{cm}^{3}\end{array}$} & \multirow{2}{*}{$\begin{array}{c}\text { Constante } \\
\text { Dielétrica } \\
1 \mathrm{MHz} \\
\end{array}$} & \multirow{2}{*}{$\begin{array}{l}\text { Condutividede } \\
\text { Térmice }\end{array}$} & \multirow{2}{*}{$\begin{array}{c}\text { Expansão } \\
\text { Térmica } \\
{ }^{\circ} C^{-1} \times 10^{-6}\end{array}$} \\
\hline & & & & & & \\
\hline$\overline{\text { Delinin }}^{\alpha}$ & 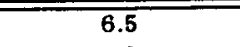 & 3 & 1.4 & 3.7 & 5.5 & 100 \\
\hline Teflon & 1.2 & 0.4 & 2.2 & 2.1 & 5.8 & 120 \\
\hline Nylon & 7 & 2.5 & 1.2 & 3.3 & 6 & 100 \\
\hline $\mathrm{Al}_{2} \mathrm{O}_{3}(99.8 \%)^{6}$ & 28 & 390 & $\overline{3.9}$ & 9 & 600 & 7 \\
\hline BN $(95 \%)^{e}$ & 4.5 & 60 & 2.08 & 4.4 & 600 & 3 \\
\hline Diamante $^{d}$ & 200 & 1000 & 3.5 & 5.5 & 18000 & 2 \\
\hline Macor $^{e}$ & 8 & 64 & 2.52 & 5.8 & 40 & 9 \\
\hline Pirex ${ }^{f}$ & 6 & 70 & 2.32 & 4.65 & 30 & 5 \\
\hline Quartzo & 5 & 72 & 2.2 & 3.9 & 33 & 0.5 \\
\hline Kel-F & 2 & 1.3 & 2.1 & 2.45 & 5 & 60 \\
\hline Kevlar $49^{\circ}$ & 270 & 117 & 1.44 & 5 & 6 & -2 \\
\hline Policarbonato & 6 & 2.5 & 1.2 & 2.8 & 4.5 & 60 \\
\hline Poliestireno & 4 & 3 & 1.05 & 2.5 & 2.5 & 70 \\
\hline Fenólice & 10 & 10 & 1.35 & 5.4 & 4 & 40 \\
\hline PMMA $^{h}$ & 7 & 3 & 1.2 & 2.75 & 4.5 & 80 \\
\hline Polipropileno & 3 & 1.2 & 0.9 & 2.25 & 5 & 110 \\
\hline Vespel SPI ${ }^{i}$ & 8 & 2.5 & 1.4 & 3.5 & 8 & 50 \\
\hline
\end{tabular}

${ }^{a}$ Polioxidometileno - Marca registrada da E. I. DUPONT DeNumours Co.

${ }^{b}$ Material experimental da Coors Ceramics, $99.8 \% \mathrm{Al}_{2} \mathrm{O}_{3}$ (Safira) extrusado.

${ }^{\complement}$ Tipo de HBN prensado a quente da Union Carbide.

dDiamante sintético da Glennel Corporation - As Características de diamante sintético variam muito de fabricante para fabricante.

'Vidro - Cerâmica maquinável da Corning Glass Works, com $55 \%$ de mica sintético fluoretado e $45 \%$ de vid zo borosilicato.

Vorning Glass com resistência até $10^{10} d y n / \mathrm{cm}^{2}$

${ }^{g}$ Fibra Aramida (Poliamida Aromático) da DUPONT.

${ }^{h}$ Acrílico.

i Resina de Poliamida - Marca registrada da E. I. DUPONT DeNumours Co.

Tabela 4.1: Propriedades mecânicas, elétricas e térmicas de alguns materiais plásticos, cerâmicos e vítreos. 


$$
p_{s}=\frac{1}{2} \omega^{2}\left[\rho_{1} r_{i}^{2}+\rho_{2}\left(r_{2}^{2}-r_{i}^{2}\right)\right]
$$

Observe que temos uma expansão de raio do rotor; porém, não podemos pensar em diminuir o raio do rotor sem antes levar em conta a expansão térmica de cada material e que a rigidez do mancal aerostático depende da folga que será influenciada pela diminuição desse raio. Para materiais cerâmicos e vítreos Doty $\&$ Ellis [1] mostrou que a diminuição do raio do rotor não afeta a estabilidade do rotor. Porém, o mesmo não é verdade para materiais plásticos que possuem uma alta expansão térmica e baixo módulo de Young. Portanto o que se faz necessário é uma otimização experimental de cada material utilizado.

\section{4:5 Nosso Rotor}

Em nosso projeto de sistema de rotação da amostra, utilizamo-nos de um sistema baseado num mancal aerostático e num sistema de propulsão idênticos aos discutidos anteriormente.

Apresentamos o projeto do sistema de rotação da amostra desenvolvido no Laboratório de Ressonância Magnética do DFCM-IFQSC-USP, figura (4.4) em três componentes principais:

- Rotor - É o compartimento que contém a amostra. Ele gira através da propulsão de suas aletas, confeccionadas na tampa desse rotor ("end cap rotor").

- "Stator" - A sustentação da amostra e seu recepiente (o rotor) é feita pelo mancal aerostático - que também serve de lubrificante de rotação desse rotor - entre o rotor e a estrutura que contém os bocais de alimentação, o "stator". Esse por sua vez possui duas entradas para alimentação. A primeira (superior) serve para produção de jatos de ar comprimido para a propulsão do rotor e a segunda (inferior) serve para a alimentação do mancal aerostático. O "stator" também serve de suporte para a bobina de RF com acesso ao exterior do sistema via saídas estabelecidas no "housing" que são parafusos que não permitem a saída do ar do compartimento do "housing".

- "Housing" - É um reservatório de ar comprimido á pressão constante para o sistema de mancal aerostático e sistema de propulsão, além de servir como sustentação mecânica do "stator".

A alimentação do ar comprimido para o mancal aerostático e propulsāo é feita através de uma fonte externa, isto é compressores de ar que não usam óleo. Esse sistema de alimentação externa deve possuir um filtro de partículas, para evitar que provoquem danos ao rotor e aletas de propulsão ou entupimentos dos pequenos furos de alimentação. Além disso possui um desumidificador para secar a água contida no ar comprimido. Para tal utilizamo-nos de um circuito de ar conforme a figura (4.5).

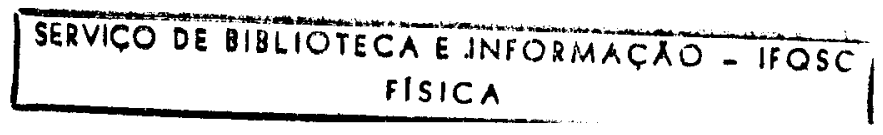




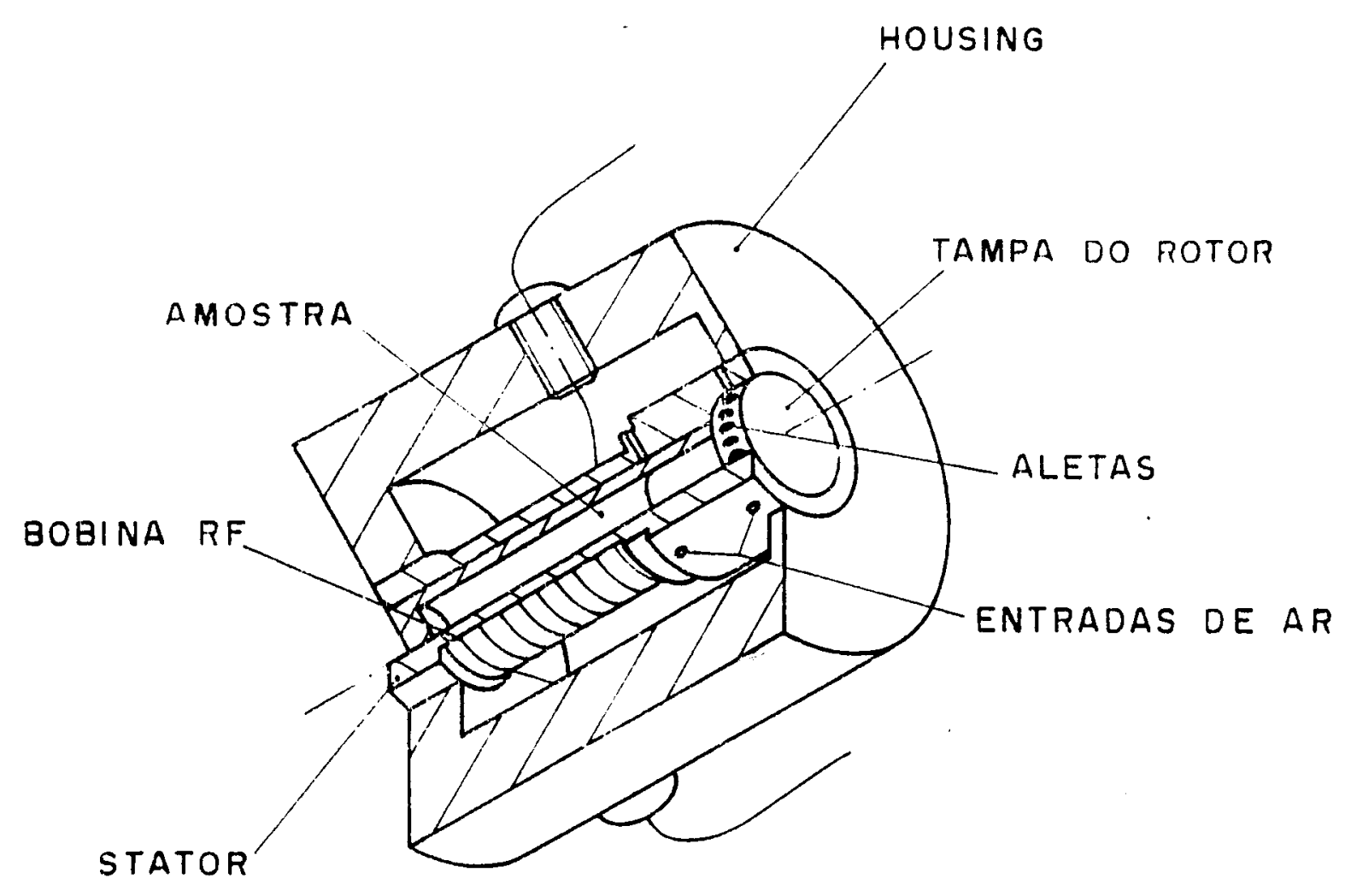

Figura 4.4: Sistema de Rotação da Amostra (LRM-DFCM-IFQSC-USP). 


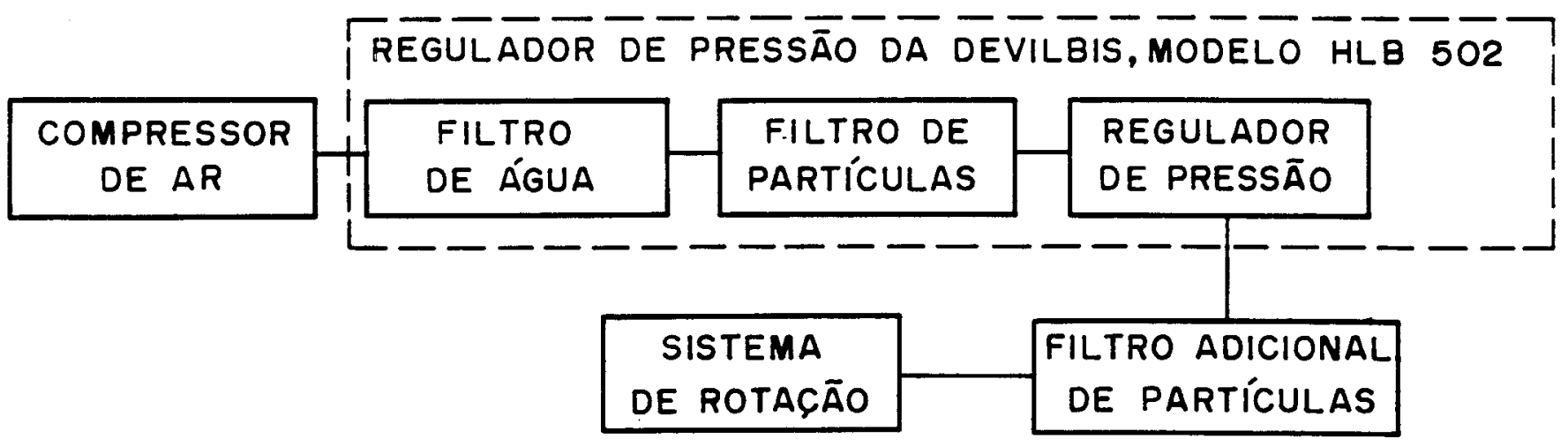

Figura 4.5: Circuito de ar comprimido.

O nosso sistema possui limitações no que diz respeito à escolha de materiais e sua usinagem. Tínhamos a nossa disposição Delrin, Teflon e Nylon, apesar de várias tentativas de importação de Nitrato de Boro (BN) e Macor. Outra limitaçāo foi a confeç̧ão desses rotores, pois contamos com uma estrutura de oficina mecânica que não é adaptada para confecções de alta precisão, com os materiais utilizados, por exemplo precisão da ordem de milésimos de milímetros.

Construímos várias versōes (três) com variaçōes no que diz respeito a:

- Material utilizado para rotor e "stator".

- Dimensões da tampa do rotor com relação ao "stator".

- Número de aletas utilizadas.

Precisamos sempre levar em conta que esses materiais para confecção dos rotores não devem conter os núcleos atômicos que estamos intéressados em medir. Além disso, procuramos escolher um material de baixa dureza para o rotor e um de alta dureza para o "stator", de modo que em caso de algum acidente ficaria mais fácil substituir o rotor do que o "stator".

Para possíveis experimentos com variação da temperatura, podemos utilizarmo-nos do próprio fluido que serve o mancal aerostático para variarmos a temperatura. Por isso a necessidade de materiais para o rotor e "stator" de baixa expansão térmica e por outro lado alta condutividade térmica para suportar grandes variaçōes de temperatura.

Como o "stator" suporta a bobina de RF e o rotor gira dentro do"stator", seus materiais também não devem possuir constantes dielétricas que introduzam capacitâncias 
espúrias no circuito de RF. Sendo altas rotaçōes nosso interesse, também se torna necessário materiais para o rotor com baixa densidade e que suporte altas tensões radiais e também conforme (4.8), alto módulo de Young.

Obtivemos, assim, uma versão final, apresentada no apêndice C, onde utilizamos-nos de Teflon para os rotores e Del-rin para o "stator" e "housing" e tampas do rotor com 13 aletas.

As medidas de freqüência de rotação dos rotores e verificação de sua estabilidade temporal foram feitas, a partir de um fototransístor, conforme o esquema demonstrado na figura (4.6). Na tampa do rotor pintamos uma faixa negra, e nela incidimos luz. O fototransístor, conta o número de incidencias dessa faixa escura, emitindo um sinal oscilante, modulado pela freqüência de repetição dessa faixa. O circuito comparador LM311 recebe esse sinal oscilante, converte-o em pulsos retangulares de $+/-12 \mathrm{~V}$, que são enviados ao osciloscópio e/ou frequencímetro que medem a freqüència de repetição registrada que resulta na freqüência de rotação do rotor.

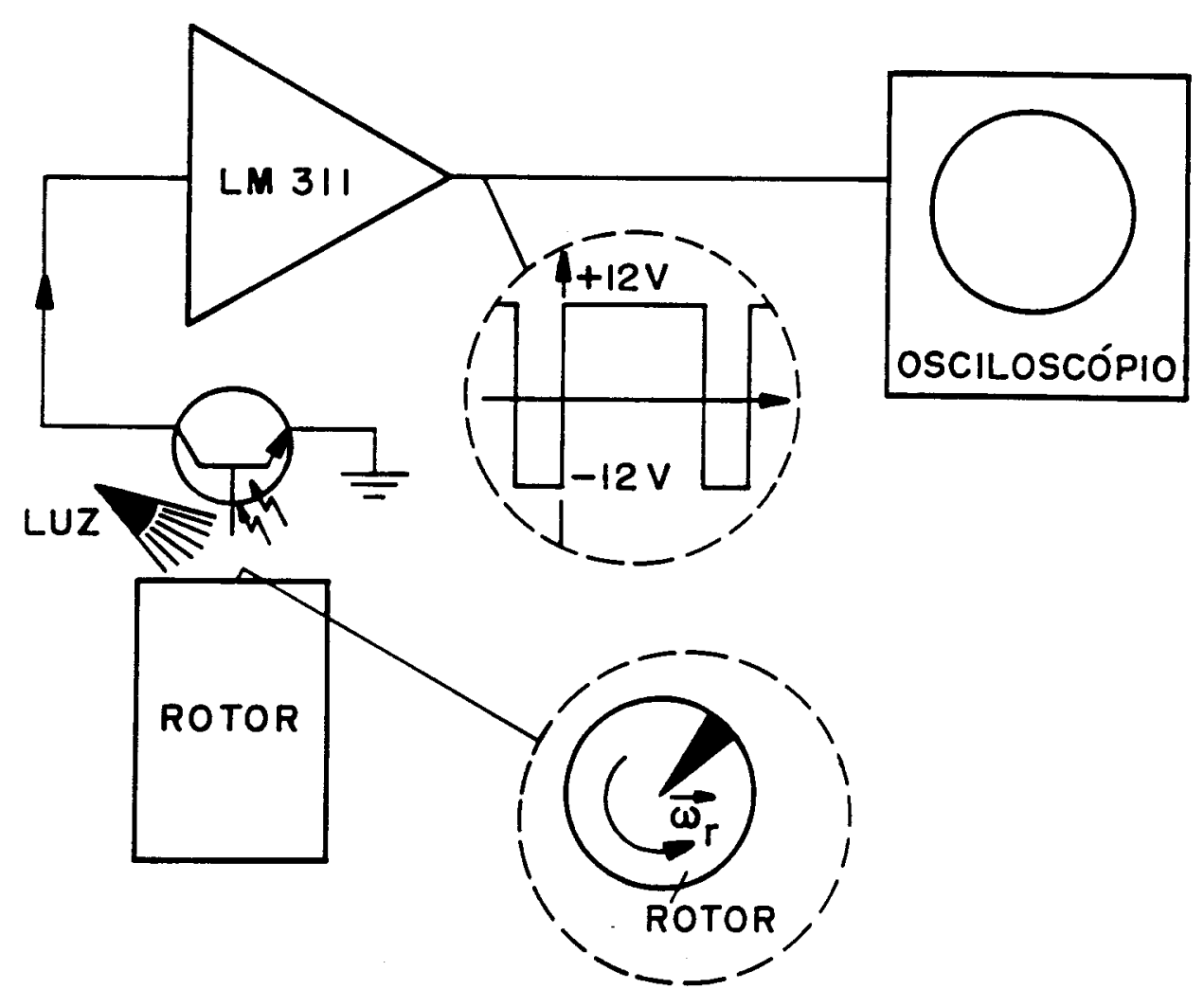

Figura 4.6: Sistema para medida da freqüência do rotor.

No próximo capítulo veremos como medir essa frequiência de rotação da amostra 
através das bandas laterais. 


\section{Bibliografia}

[1] F. David Doty and Paul D. Ellis, Design of High Speed Cilindrical NMR Sample Spinners, Rev. Sci. Instrum. 52(12),1868-1875, (1981).

[2] E. Andrew, L. Farnell, M. Firth, T. Gladhill and I. Roberts, J. Magn. Reson. 1,27 (1969). Uma versão desse projeto menos susceptível a vibrações: V. J. Bartuska end G. E. Maciel, J. Mag. Reso. 42,312 (1891).

[3] E. R. Andrew, in Philosophical Transactions of The Royal Society of London - Mathematical and Physical Sciences - Nuclear Magnetic Resonance Spectroscopy in Solids, 299, 505-520, No 1452, (1981).

[4] J. W. Powell, Design of Aerostatic Bearings, The Machinery Publishing. London (1970).

[5] B. M. Purquerio, Banco de Ensaio para Mancais Aerostáticos Axiais Projeto Construção e Testes Experiementais., Tese de Mestrado - EESC-USP, (1975).

[6] Manual da Bruker - "High Resolution NMR of Solids".

[7] T. J. Bonagamba, Relatório de período probatório 88/90 - IFQSC-USP, Projeto: Espectroscopia de Alta Resolução em Sólidos por RMN.

[8] A. Samoson and A. Pines, Double Rotor for Solid-State NMR, Rev. Sci. Instrum. 60 (10), 1989, (Artigo de Qualificação).

[9] E. R. Andrew, W. S. Hinshaw, M. G. Hutchins and-A. Jasinski, Proc. R. Soc. Lond., A364, 553-567, (1978).

[10] D. F. Wilcock and E. R. Booser, Bearing Design and Application, McGraw-Hill Book Company, Inc. First Edition,(1957).

[11] A. H. Shapiro, The Dynamics and Thermodynamics of Compressible Fluid Flow, Vol. 1, The Ronald Press Company, New York, (1953).

[12] L. Landau et E. Lifchitz, Mécanique des Fluides, Cours de Physique Théorique,Tome VI, Editions Mir, Moscou (1971). 
[13] V. M. S. Gil, C. F. Geraldes, Ressonância Magnética Nuclear, F. Calouste Gulbenkian, (1987). 


\section{Capítulo 5}

\section{Resultados Experimentais}

\subsection{Apresentação}

Nossa intenção nesse trabalho foi o desenvolvimento instrumental da técnica de rotação da amostra em torno do ângulo mágico, para isso construímos e caracterizamos rotores para rotação da amostra, (apêndice $\mathrm{C}$ ).

Porém, para o aprimoramento dessa técnica realizamos vários testes procurando a otimização e estabilidade da rotação da amostra. Para tal, utilizamo-nos de amostras sólidas com núcleos de spin $\frac{1}{2}$ que não apresentassem larguras de linha muito grandes.

A sequência de pulsos indicada na figura (5.1), foi utilizada para a geração dos ecos rotacionais.

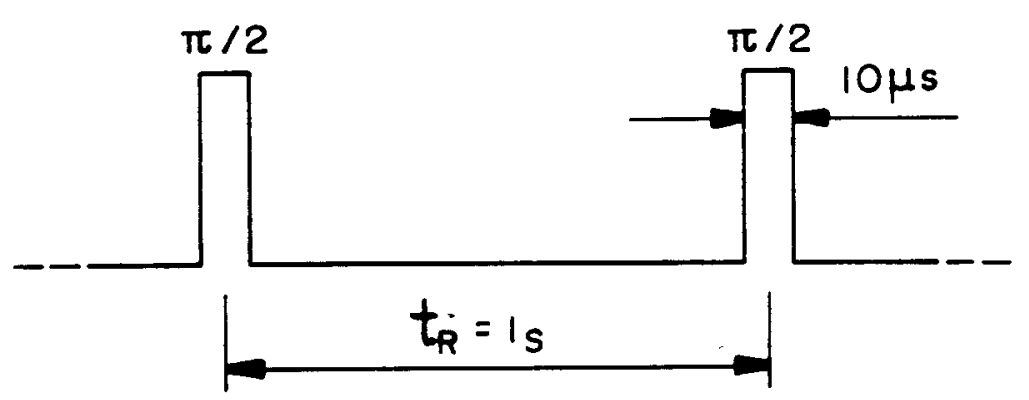

Figura 5.1: Sequência de pulsos utilizada em nosso experimento.

\subsection{Bandas Laterais \& Ecos Rotacionais}

Para determinar a freqüência de rotação da amostra via bandas laterais e/ou ecos rotacionais utilizamo-nos de núcleos de hidrogenio presentes em amostras sólidas onde a 
largura de linha não fosse muito grande. Isto se fez necessário pois o sinal de RMN será muito melhor e compatível com a rotação do rotor, que gira em torno de $500 \mathrm{~Hz}$, determinada a partir do esquema com o fototransístor apresentado no capítulo anterior.

Procuramos satisfazer tais necessidades, utilizando como amostra pó bem fino de semente de soja, empacotado dentro do compartimento para amostra do rotor. Essa amostra apresenta um espectro de $H^{1}$ com largura de linha em torno de de $670 \mathrm{~Hz}$, obtido experimentalmente conforme figura (5.2), onde não utilizamos da rotação da amostra em torno do ângulo mágico.

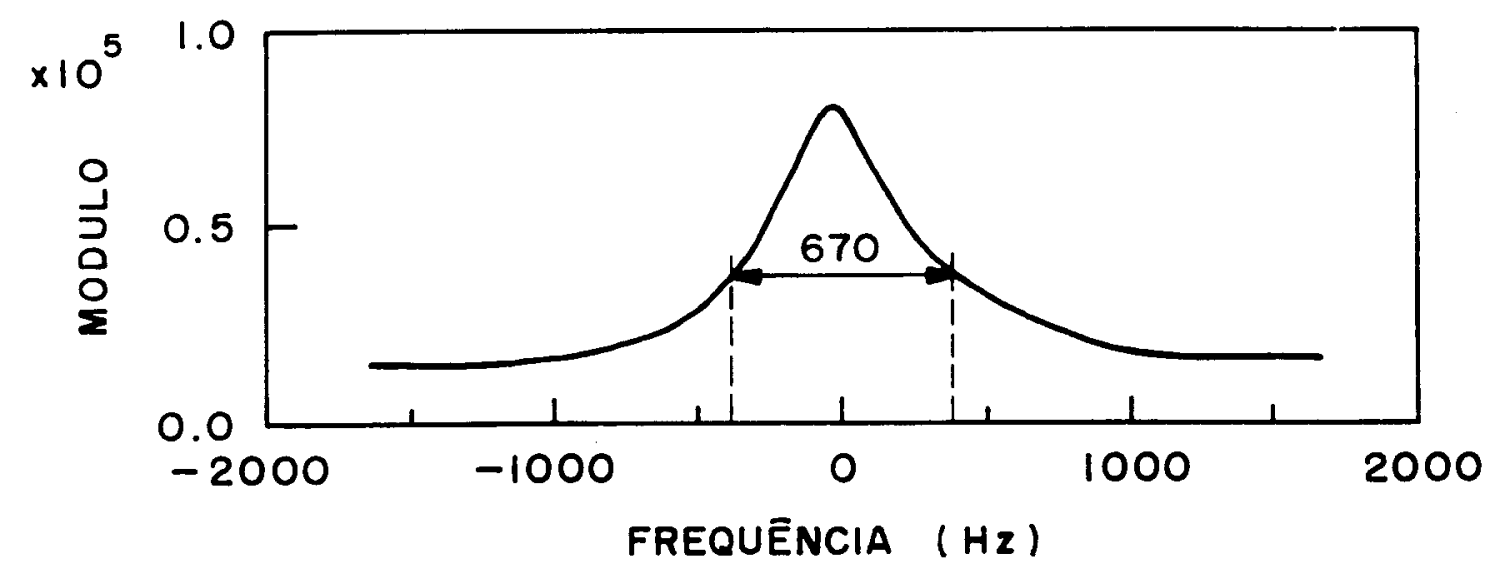

Figura 5.2: Espectro de RMN, para amostra de soja sem RAAM para o núcleo de $H^{1}$.

Utilizando o sistema de rotação, giramos a amostra em torno do ângulo mágico, porém, com freqüência menor que $670 \mathrm{~Hz}$, resultando num sinal de RMN com ecos rotacionais, conforme equação (2.45) \& (figura (5.3), e por sua vez, temos um espectro com bandas laterais, conforme (2.46) \& (figura (5.4).

Do sinal de RMN com ecos rotacionais podemos obter o período de rotação da amostra $T_{r}$, medindo-se a separação entre os ecos rotacionais. Por sua vez, do espectro de RMN com bandas laterais, obtemos a freqüência de rotação da amostra $\nu_{r}$ medindo-se a separação entre as bandas laterais. A partir desse procedimento, obtemos a curva de calibração do sistema de rotação medindo-se $\nu_{r}$ ou $T_{r}$ em função da pressão $\mathrm{P}$ do sistema de ar comprimido que alimenta o sistema de rotação. Isso está mostrado na figura (5.5).

\subsection{Desacoplamento em Alta Potência \& RAAM}

Além da técnica de rotação em torno do ângulo mágico, existe outra técnica que também propicía-nos estreitamento de linhas. Somente depois do desenvolvimento da combinação das duas técnicas simultaneamente em apenas um experimento é que se tornou possível a 

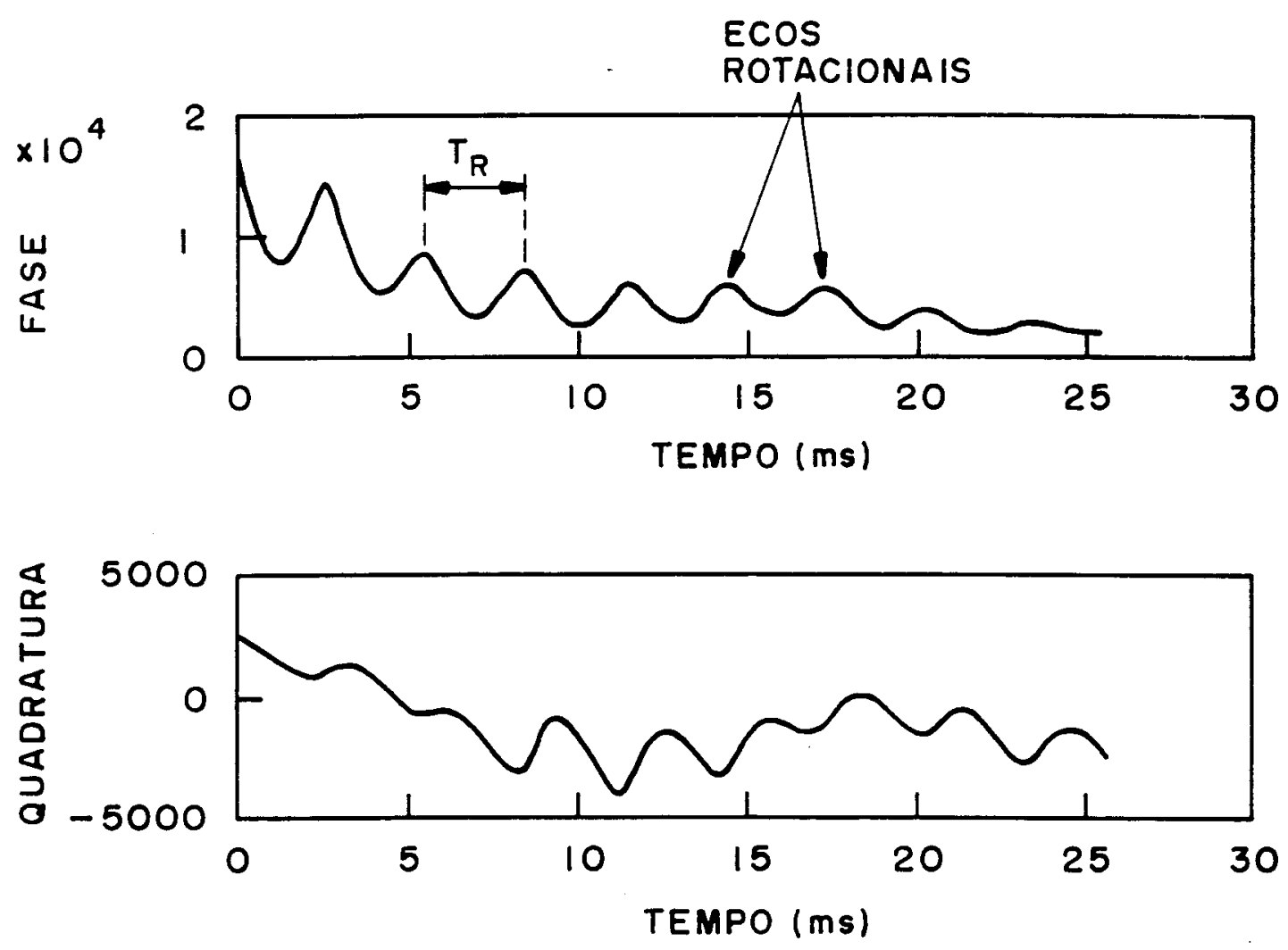

Figura 5.3: Sinal de RMN em fase e quadratura com Ecos Rotacionais após 16 médias, resultando num período de rotação da amostra de soja, $T_{R} \simeq 3 \mathrm{~ms}$. 


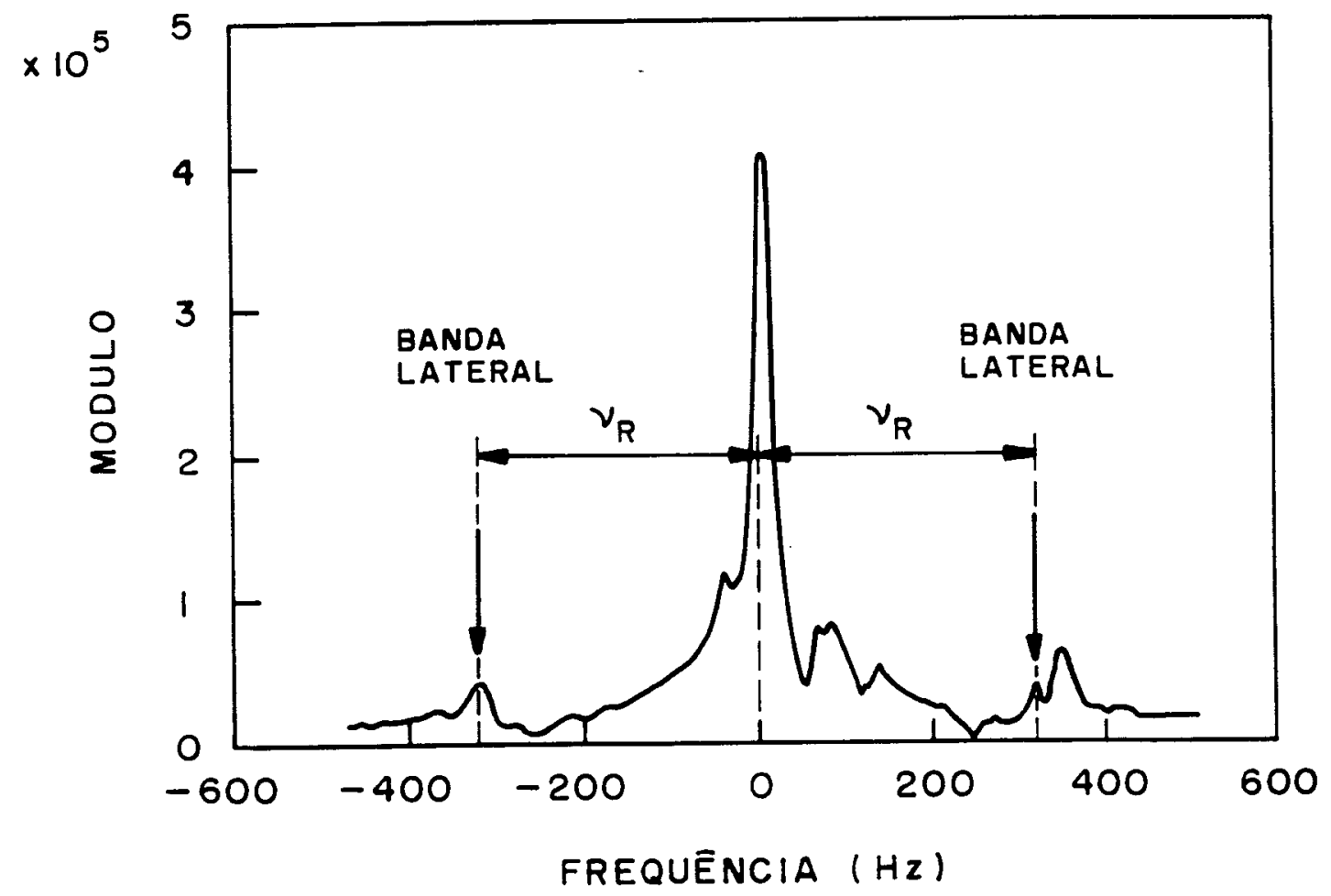

Figura 5.4: Espectro de RMN com Bandas Laterais, após 16 médias, resultando para a freqüência de rotação da amostra de soja, $\nu_{\mathrm{r}} \simeq 323 \mathrm{~Hz}$. 


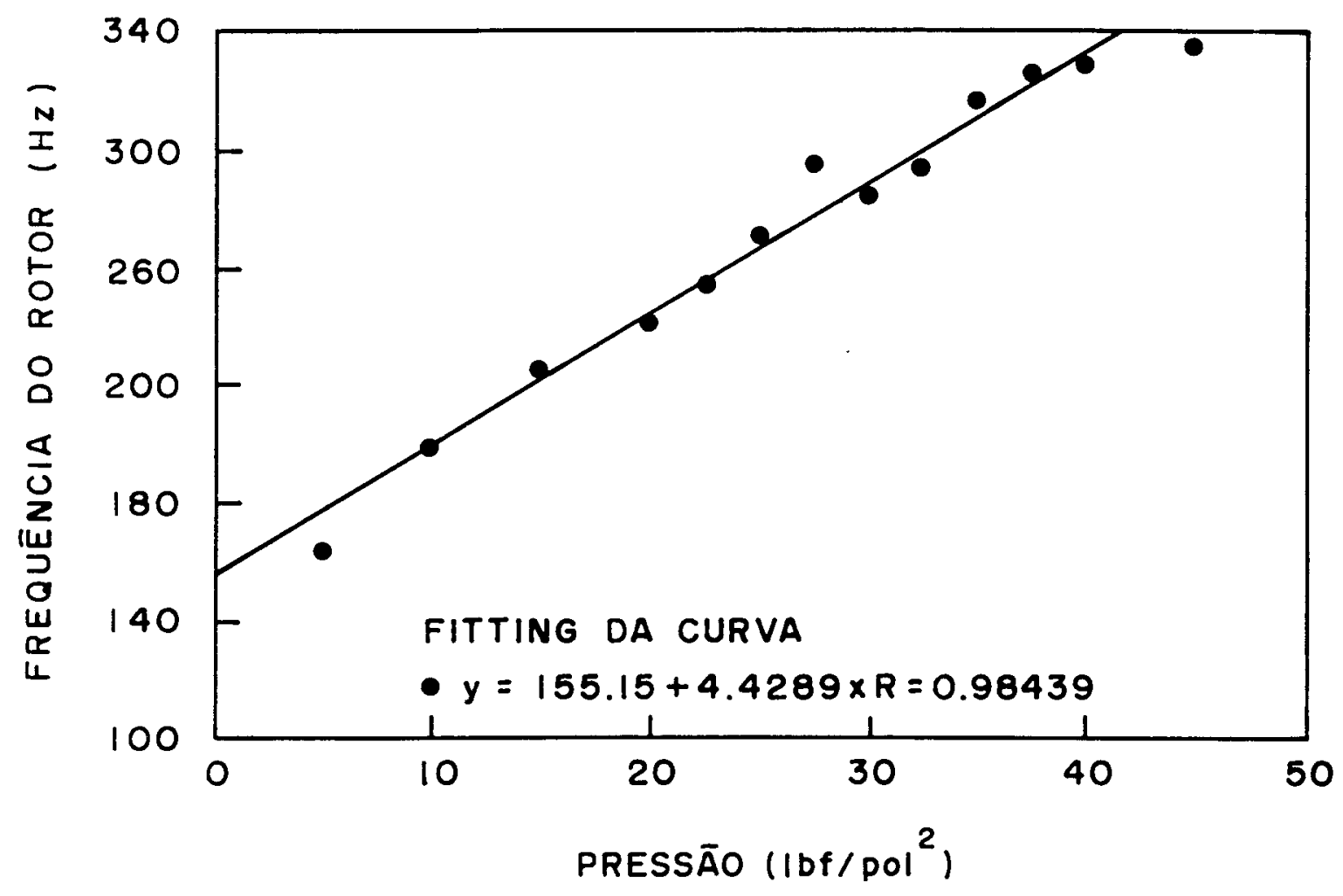

Figura 5.5: Curva de calibração do sistema de rotação da amostra. 
obtenção de espectros de alta resolução em sólidos por RMN em nucleos como, $C^{13}, N^{15}$, $P^{31}$, etc.

Quando trabalhamos com núcleos de $C^{13}$ em amostras sólidas observamos que seus espectros são alargados fundamentalmente pela interação dipolar heteronuclear $(\sim k H z)$. O campo magnético adicional local que surge é devido à presença de núcleos vizinhos mais abundantes como, por exemplo, prótons em sólidos orgânicos. É possível desacoplar os núcleos de $C^{13}$ dos prótons vizinhos. Esse desacoplamento é feito via redução do momento magnético efetivo do próton-pela aplicação de um campo de radiofreqüência (RF) $\mathbf{B}_{2}$ que mantém o momento de dipolo magnético $(\mu)$, do mesmo em alta rotação, figura (5.6). Observe que o valor médio de $\mu$ torna-se nulo, $\langle\mu\rangle=0$.

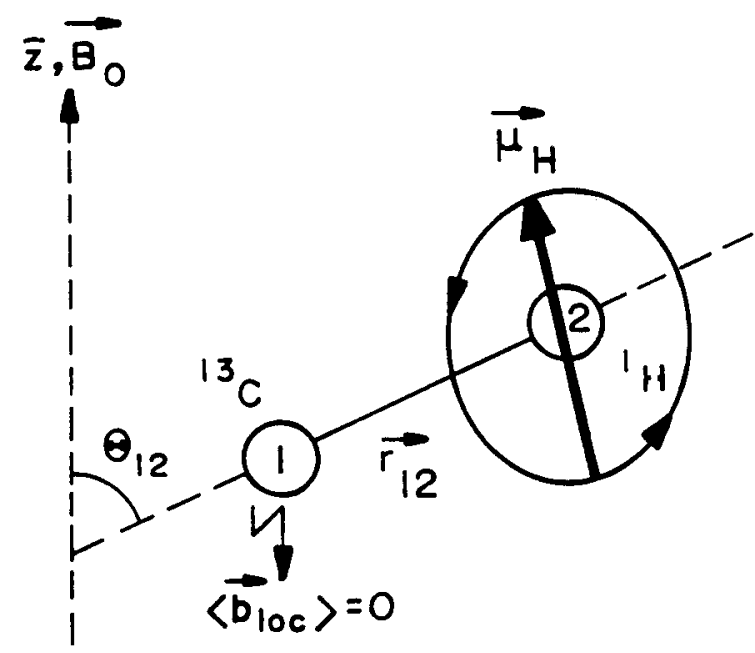

Figura 5.6: Efeito do desacoplamento. Devidoa rápida rotação do momento magnético $(\mu)$, do núcleo $H^{1}$ o campo local $\left(b_{l o c}\right)$, é reduzido a zero, em média para o $C^{13}$.

Para o nosso experimento, utilizamo-nos de amostras sólidas de adamantano, com estrutura química apresentada na figura (5.7), onde observamos a existência de dois grupos de $C^{13}$ com vizinhança diferentes, 6 grupos $\mathrm{CH}_{2}$ e 4 grupos $\mathrm{CH}$. Eliminando-se as anisotropias de interação dipolar e deslocamento químico anisotrópico através de RAAM e DAP, teremos um espectro com duas linhas bem distintas entre si, definidas pelo deslocamento químico isotrópico distinto para cada grupo $\left(\mathrm{CH} \& \mathrm{CH}_{2}\right)$ com relação de intensidades de 6:4. Caso contrário seria muito difícil observar o sinal, pois vimos que a largura de linha dipolar é da ordem de $\mathrm{kHz}$.

Como a anisotropia de deslocamento químico é pequena para o adamantano, somente a utilização de desacopalmento em alta potência, bastaria para obter o espectro de deslocamento químico resolvido como mostramos na figura (5.8)-(a) Porém se utilizarmos também da técnica RAAM observamos um melhor estreitamento da linha. Isso devido a eliminação da pequena anisotropia de deslocamento químico e a contribuição da RAAM na eliminação da anisotropia devido a interação dipolar, vide figura (5.7)-(b). 


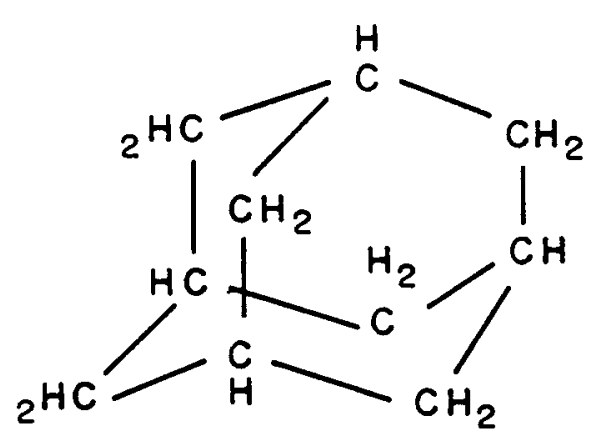

Figura 5.7: Estrutura química do Adamantano.

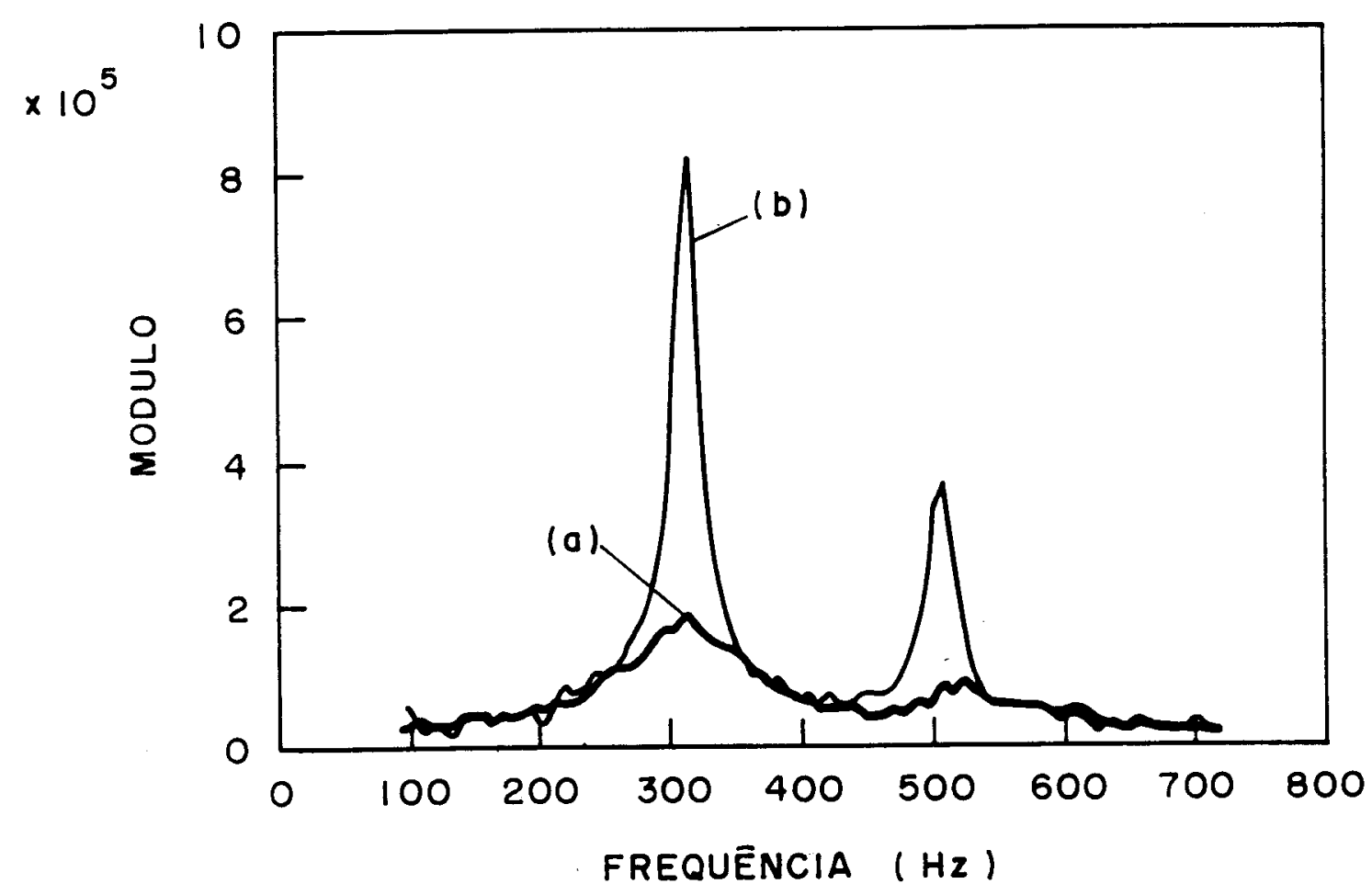

Figura 5.8: Espectro do Adamantano após 50 médias, sendo (a) apenas com DAP e (b) com $D A P$ e $R A A M$. 
CAPíTULO 5. RESULTADOS EXPERIMENTAIS 


\section{Conclusão}

A implementação e desenvolvimento de instrumentação da Técnica de Rotação da Amostra em torno do Ângulo Mágico, foi concluída - depois de três versões - com a construção de rotores com mancais aerostáticos de alta rotação, conforme apêndice C.

Obtivemos uma rotação baixa devido fundamentalmente aos seguintes fatores:

- Falta de material adequado para sua execução, ao invés de Delrin e Teflon, deveríamos ter trabalhado, por exemplo, com Macor e NB. Isso não foi possível, mesmo após várias tentativas de importação.

- Nesses projetos estivemos envolvidos com alta precisão da ordem de milésimos de milímetros, porém o maquinário que tinhamos a disposição não correspondeu à precisão requerida. Por exemplo, para confecção de furos da ordem de milésimos de milímetros, utiliza-se de laser e mesmo a confecção de superfícies de alta precisão envolve a necessidade de retificadoras e tornos mecânicos ultra-precisos.

Porém, a experiência adquirida na técnica RAAM foi eficiente, tornando possível a espectroscopia de alta resolução em amostras sólidas orgânicas, com núcleos de $C^{13}$ e $H^{1}$, com bandas estáticas da ordem de $500-1000 \mathrm{~Hz}$, que foi a faixa de rotação do nosso rotor. Exemplos de núcleos de $H^{1}$ em soja e $C^{13}$ em adamantano são apresentados nos nossos resultados experimentais.

O grupo de Ressonância Magnética - DFCM-IFQSC-USP está atualmente estabelecendo cooperação para posterior desenvolvimento do projeto de RAAM com um grupo do Departamento de Engenharia Mecânica da EESC-USP, especializado em Engenharia de Mancais Aerostáticos. 


\section{Apêndice A}

\section{Rotações}

\section{A.1 Introdução}

Estaremos aqui interessados na conexão entre momentum angular de um sistema e as propriedades de transformação desse sistema sujeito a rotação. Estas transformaçōes são expressas em termos do que acontece com a função de onda do sistema quando sıjeito a rotação. Para tal consideramos a rotação de coordenadas desse sistema de um ângulo $\theta \mathrm{em}$ torno de um eixo dado pela direção $\hat{n}$, um vetor unitário. A função de onda $\psi$ no sistema original é relacionada com a função de onda $\psi^{\prime}$ no sistema rodado pela transformação unitária ${ }^{1}$ :

$$
\begin{aligned}
\psi^{\prime} & \equiv \mathcal{R}(\hat{\mathbf{n}}, \theta) \psi, \\
\mathcal{R}(\hat{\mathbf{n}}, \theta) & =e^{-\boldsymbol{i} \boldsymbol{S}(\mathbf{n}, \theta)},
\end{aligned}
$$

sendo que $S(\hat{\mathbf{n}}, \theta) \rightarrow 0$ para $\theta \rightarrow 0$, resultando em $\mathcal{R}(\hat{\mathbf{n}}, \theta) \rightarrow 1$.

A transformação unitária $\mathcal{R}(\hat{\mathbf{n}}, \theta)$ depende de três ângulos: dois para definir a direção de $\hat{n}$ e o ângulo $\theta$ que define a magnitude da rotação. Considerando-se transformaçōes unitárias infinitesimais, isto é, desprezando os termos de ordem maior que um, para a expansāo de $\mathcal{S}(\hat{\mathbf{n}}, \theta)$, resulta:

$$
\mathcal{R}(\hat{\mathbf{n}}, \theta) \psi=(1-i \mathcal{S}(\hat{\mathbf{n}}, \theta)) \psi
$$

Definimos os operadores momento angular em termos de propriedades de transformaçōes da função de onda sujeita a rotaçōes do sistema de coordenadas. Observe que essas propriedades são independentes da magnitude do ângulo $\theta$ de rotação.

Para rotações em torno dos eixos $x, y$ e $z$ resulta:

$$
\begin{aligned}
& \mathcal{R} \psi-\psi=-i \theta \mathbf{J}_{z} \psi \\
& \mathcal{R} \psi-\psi=-i \theta \mathbf{J}_{y} \psi \\
& \mathcal{R} \psi-\psi=-i \theta \mathbf{J}_{x} \psi
\end{aligned}
$$

\footnotetext{
${ }^{1}$ Transformação unitária é uma transformação homogênea linear que preserva os comprimentos e ângulos envolvidos, isto é o produto escalar permanece invariante sob tal rotação.
} 
Os três operadores Hermitianos $\mathbf{J}_{x}, \mathbf{J}_{y}$ e $\mathbf{J}_{z}$ são operadores para as três componentes cartesianas do momentum angular. Agora para um eixo de rotação arbitrário $\hat{n}$ temos:

$$
\mathcal{R} \psi-\psi=-i \theta(\hat{\mathbf{n}} \cdot \mathbf{J}) \psi
$$

sendo $\theta$ o ângulo de rotação que de (A.3) resulta, $S=(\hat{\mathbf{n}} \cdot \mathbf{J}) \theta$, sendo o momentum angular J definido pela equação:

$$
\mathcal{R}(\hat{\mathbf{n}}, \dot{\theta}) \psi=e^{-i \theta(\hat{\mathbf{n}} \cdot \mathbf{J})} \psi .
$$

Assim, o momentum angular determina a propriedade de transformação de um sistema sujeito a rotação de suas coordenadas e inversamente, o operador $\mathbf{J}$ do momentum angular pode ser determinado pelas propriedades de rotação do sistema.

\section{A.2 Matriz dos Operadores Rotação}

A função de onda $\psi_{i j}$ que diagonaliza $\mathbf{J}^{2}$ e uma componente do momentum angular $\mathbf{J}$, com autovalores $j(j+1)$ e $m$ respectivamente, pressupoẽ a escolha de um eixo de quantização, pois $m$ é autovalor da componente do momentum angular sobre esse eixo. Desde que $\mathbf{J}^{2}$ comuta com o operador rotação:

$$
\left[\mathbf{J}^{2}, e^{(-i \theta \hat{\mathbf{n}} \cdot \mathbf{J})}\right]=\sum_{v} \frac{1}{v !}(-i \theta)^{v}\left[\mathbf{J}^{2},(\hat{\mathbf{n}} \cdot \mathbf{J})^{v}\right]=0,
$$

resulta que $\mathbf{J}^{2}$ comuta com cada elemento da série que expande $e^{(-i \theta \mathbf{n} \cdot \mathbf{J})}$. Sendo a função rodada (A.8) autofunção de $\mathbf{J}^{2}$ com autovalor invariante $j(j+1)$, temos:

$$
\left.\mathbf{J}^{2} e^{(-i \theta \mathbf{n} \cdot \mathbf{J})} \psi_{j m}=j(j+1) e^{(-i \theta \hat{\mathbf{n}} \cdot \mathbf{J}}\right) \psi_{j m} .
$$

Isso expressa o fato de o momentum angular total $\mathbf{J}$ não se importar com a qual direção rodamos ou mesmo com qual sistema de coordenadas estamos referindo-nos.

Por sua vez essa mesma função rodada não diagonaliza $\mathbf{J}_{z}$, resultando numa superposição de autofunções $\psi_{j m^{\prime}}$ com diferentes projeções de número quântico $m^{\prime}$ mas com o mesmo momentum angular total $j$ :

$$
\mathcal{R} \psi_{j m}=\sum_{m^{\prime}}\left\langle j m^{\prime}\left|e^{(-i \theta \hat{\mathbf{n}} \cdot \mathbf{J})}\right| j m\right) \psi_{j m^{\prime}}
$$

Resulta assim para a rotação em torno do eixo de quantização, $\hat{\mathbf{n}} \cdot \mathbf{J}=J_{z}$ :

$$
\left\langle j m^{\prime}\left|e^{(-i \theta \hat{\mathbf{n}} \cdot \mathbf{J})}\right| j, m\right\rangle=\delta_{m, m^{\prime}} e^{-i m \theta},
$$

observe que o número quântico projetado permanece o mesmo, mudando somente a fase de acordo $\operatorname{com} \mathcal{R} \psi_{j m}=e^{-i m \theta} \psi_{j m}$.

No caso geral precisamos calcular os elementos da matriz que representa o operador rotação, $\mathcal{R} \psi_{j m}=e^{(-i \theta \hat{\mathbf{n}} \cdot \mathbf{J})}$, dado por, $\left\langle j m^{\prime}\left|e^{(-i \theta \hat{\mathbf{n}} \cdot \mathbf{J})}\right| j m\right\rangle$. Vimos que é necessário três 
parâmetros para especificar a rotação, isto é as três componentes do vetor $(\theta \hat{n})$, onde $\theta$ é a magnitude e $\hat{n}$ é a direção da rotação dada pelos ângulos de Euler, os quais são denominados por $\alpha, \beta$ e $\gamma$, que são definidos por três passos sobre o sistema originaı $x, y$ e $z$ assim descritos:

1. Rotação de $\alpha$ sobre $z$, definindo $x^{\prime}, y^{\prime}, z^{\prime}$, fig. 1(a).

2. Rotação de $\beta$ sobre $y^{\prime}$, definindo $x^{\prime \prime}, y^{\prime \prime}, z^{\prime \prime}$, fig. 1(b).

3. Rotação de $\gamma$ sobre $z^{\prime \prime}$, definindo $x^{\prime \prime \prime}, y^{\prime \prime \prime}, z^{\prime \prime \prime}$, fig. 1(c).

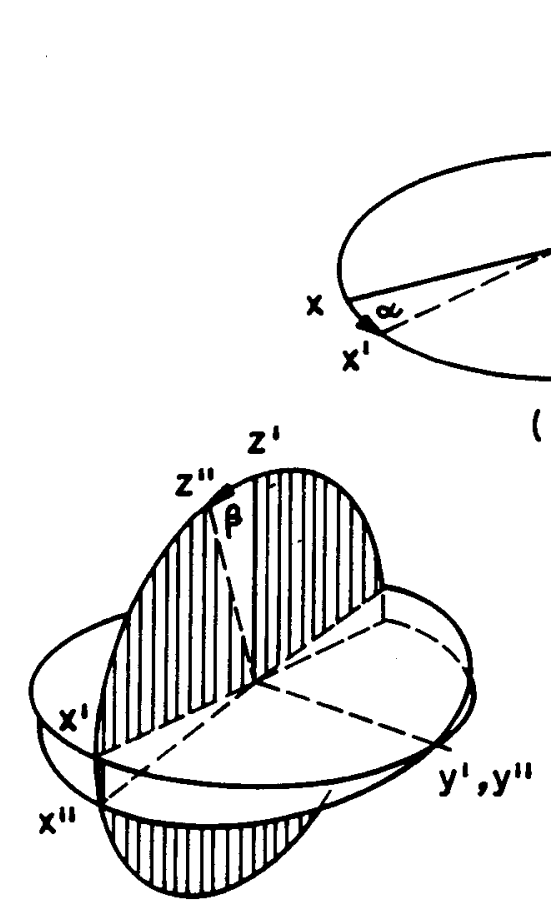

(b)

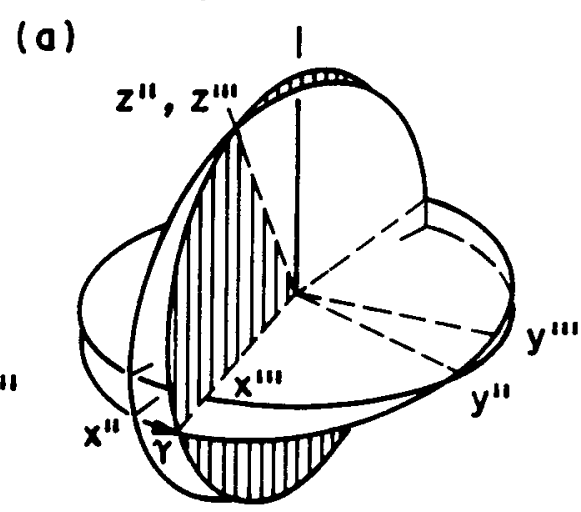

(c)

Figura A.1: Ângulos de Euler $\alpha, \beta$ e $\gamma$ e as três rotacões que carregam o sistema de coordenadas iniciais $(x, y, z)$ para o sistema final $\left(x^{\prime \prime \prime}, y^{\prime \prime \prime}, z^{\prime \prime \prime}\right)$.

Resultando:

$$
e^{-i \boldsymbol{\theta}(\mathbf{n} \cdot \mathbf{J})}=\mathcal{R}_{\gamma} \mathcal{R}_{\boldsymbol{\beta}} \boldsymbol{R}_{\boldsymbol{\alpha}}=e^{-i \boldsymbol{\gamma} \mathbf{J}_{z^{\prime \prime}}} e^{-i \beta \mathbf{J}_{y^{\prime}}} e^{-i \alpha \mathbf{J} \mathbf{z}},
$$

onde $\mathbf{J}_{y^{\prime}}$ e $\mathbf{J}_{z^{\prime \prime}}$ são as componentes de $\mathbf{J}$ ao longo de $y^{\prime}$ e $z^{\prime \prime}$.

Observe que $\mathcal{R}_{\boldsymbol{\beta}}=e^{-i \beta \mathbf{J}_{y^{\prime}}}$ é a transformação de $e^{-i \alpha J_{z}}$ que carrega o eixo $y$ para o eixo $y^{\prime}$, sob uma prévia rotação $R_{\alpha}=e^{-i \alpha J_{x}}$. Lembrando-se de que uma transformação unitária $\mathcal{U}$ transforma um operador $\Omega$ em $\Omega^{\prime}=U \Omega U$, isto é, $e^{-i \beta \mathbf{J}_{y^{\prime}}}=e^{-i \alpha \mathbf{J}_{z}} e^{-i \beta \mathbf{J}_{y}} e^{i \alpha \mathbf{J}_{z}}$. Por sua vez $\mathcal{R}_{\gamma}=e^{-i \gamma \mathbf{J}_{z^{\prime \prime}}}$ é a transformação de $e^{-i \gamma \mathbf{J}_{z^{\prime}}}$ sob rotação $\mathcal{R}_{\beta}=e^{-i \beta \mathbf{J}_{y^{\prime}}}$ carregando $z^{\prime}$ em $z^{\prime \prime}$, vindo $\mathcal{R}_{\gamma}=e^{-i \gamma \mathbf{J}_{z^{\prime \prime}}}=e^{-i \beta \mathbf{J}_{y^{\prime}}} e^{-i \gamma \mathbf{J}_{z^{\prime}}} e^{i \beta \mathbf{J}_{y}}$. 
Substituindo $\mathcal{R}_{\gamma}$ e $\mathcal{R}_{\beta}$ em (A.13), resulta finalmente:

$$
\mathcal{R}=e^{-i \boldsymbol{\alpha} \mathbf{J}_{z}} e^{-i \beta \mathbf{J}_{\mathbf{y}}} e^{-i \boldsymbol{\gamma} \mathbf{J}_{z}},
$$

essa equação expressa o fato de que a rotação $R$ é realizada segundo tres ângulos de Euler sucessivas, conforme os items 1,2 e 3 definido anteriormente. Observe que os eixos de rotações são eixos de diferentes sistemas de coordenadas obtidos pelas rotações sucessivas. Porém essas rotações podem ser levadas num mesmo sistema de coordenadas se as ordens de rotações forem invertidas, isto é, primeiro uma rotação de $\gamma$ é feita sobre o eixo $z$, depois uma rotação $\beta$ é feita sobre o eixo $y$ e finalmente, uma rotação $\alpha$ é feita sobre o eixo $z$ :

$$
R \leftarrow \underbrace{e^{-i \alpha J_{z}}}_{\text {sobre } z} \leftarrow \underbrace{e^{-i \beta J_{y}}}_{\text {sobre y }} \leftarrow \underbrace{e^{-i \gamma J-z}}_{\text {sobre } z} .
$$

Podemos agora calcular a depêndencia que o operador rotação possui de $\alpha, \beta$ e $\gamma$ seguido a convenção de Wigner, (A.11), definimos a matriz $\mathcal{D}_{m^{\prime}, m}^{(j)}(\alpha \beta \gamma)$ :

$$
\mathcal{R} \psi_{j m}=\sum_{m^{\prime}} \mathcal{D}_{m^{\prime}, m}^{(j)}(\alpha \beta \gamma) \psi_{j m^{\prime}}
$$

Por sua vez de (A.11) e (A.14) vem:

$$
\mathcal{D}_{m^{\prime}, m}^{(j)}(\alpha \beta \gamma)=e^{-i \alpha m^{\prime}}\left\langle j m^{\prime}\left|e^{-i \beta \mathbf{J}_{y}}\right| j m\right) e^{-i \gamma m}
$$

que são os elementos da matriz de Wigner dos operadores de rotação.

\section{A.3 Determinação das Matrizes de Rotação}

Vimos que quando rodamos um sistema de coordenadas segundo os ângulos de Euler $\mathcal{R}(\alpha \beta \gamma)$, cada autofunção do operador momentum angular transforma-se segundo $\mathcal{D}_{m^{\prime}, m}^{(j)}(\alpha \beta \gamma)$. Por sua vez utilizando-se da série inversa de Clebsh-Gordon ${ }^{2}$ dada por:

$$
\begin{aligned}
\mathcal{D}_{\mu, m}^{(j)}= & \sum_{\mu_{1}} \sum_{m_{1}} C\left(j_{1} j_{2} j ; m_{1}, m-m_{1}\right) C\left(j_{1} j_{2} j ; \mu_{1}, \mu-\mu_{1}\right) \\
& \times \mathcal{D}_{\mu_{1}, m_{1}}^{\left(j_{1}\right)} \mathcal{D}_{\mu-\mu_{1}, m-m_{1}}^{\left(j_{2}\right)} \text { sendo } \mu=\mu_{1}+\mu_{2},
\end{aligned}
$$

poderemos construir as matrizes- $\mathcal{D}^{(j)}$ com elementos $\mathcal{D}_{\mu, m}^{(j)}$ para um momentum angular arbitrário $j$ para os quais os momentos angulares $j_{1}$ e $j_{2}$ satisfaçam:

$$
\Delta\left(j_{1}, j_{2}\right) \Rightarrow\left|j_{1}-j_{2}\right| \leq j \leq j_{1}+j_{2} \quad \text { (Regra do Triângulo) }
$$

\footnotetext{
${ }^{2}$ pag. 58 da referência [1]
} 
Inicialmente determinamos $\mathcal{D}_{m^{\prime}, m}^{(1)}$ a partir das propriedades de transformaçōes sob rotaçōes das autofunçōes de um sistema com momentum angular $j=1$, para tal utilizamosnos dos harmônicos esféricos $Y_{1, m}, m=0,1,-1$, que são autofunções do momentum angular orbital para $\ell=1$. A razão para essa escolha é que $Y_{1, m}$, que da a posição de um ponto $P$ sobre a esfera unitária, possui propriedades de transformações do sistema de coordenadas cartesianas $(x, y, z)$ para o sistema de coordenadas esférico $(r, \theta, \varphi)$, dado por:

$$
Y_{1, m}(\theta, \varphi)=\left(\frac{3}{4 \pi}\right)^{\frac{1}{2}} \frac{1}{r}\left(\begin{array}{cl}
-\frac{1}{\sqrt{2}}(x+i y), & m=1 \\
z, & m=0 \\
\frac{1}{\sqrt{2}}(x-i y), & m=-1
\end{array}\right) .
$$

Em particular, o vetor do ponto $P$ nas bases esféricas será:

$$
\mathbf{r}_{s}=\left(\begin{array}{c}
-\frac{1}{\sqrt{2}}(x+i y) \\
z \\
\frac{1}{\sqrt{2}}(x-i y)
\end{array}\right) .
$$

Se o sistema de coordenadas é rodado através dos ângulos de Euler $(\alpha, \beta, \gamma)$, as coordenadas $(x, y, z)$ passam para $\left(x^{\prime}, y^{\prime}, z^{\prime}\right)$, sendo conectados por $\mathcal{M}(\alpha, \beta, \gamma)$ onde $\mathbf{r}^{\prime}=\mathcal{M} \mathbf{r}$ e por sua vez o vetor $\mathbf{r}_{s}^{\prime}$ para o ponto $P$, será:

$$
\mathbf{r}_{s}^{\prime}=\left(\begin{array}{c}
-\frac{1}{\sqrt{2}}\left(x^{\prime}+i y^{\prime}\right) \\
z^{\prime} \\
\frac{1}{\sqrt{2}}\left(x^{\prime}-i y^{\prime}\right)
\end{array}\right) .
$$

Observe que de (A.21) e (A.22) resulta $\mathbf{r}_{s}^{\prime}=\mathcal{M}, \mathbf{r}_{s}$. Agora se multiplicarmos $\mathbf{r}_{s}^{\prime}$ e $\mathbf{r}_{s}$ pelo invariante $\left(\frac{3}{4 \pi}\right)^{\frac{1}{2}} \frac{1}{\tau}$, temos as propriedades de transformaçōes dos esféricos harmônicos de primeira ordem:

$$
Y_{1 m}\left(\theta^{\prime}, \varphi^{\prime}\right)=\sum_{m^{\prime}}\left(\mathcal{M}_{s}\right)_{m m^{\prime}} Y_{l m^{\prime}}(\theta, \varphi)
$$

Porém da definição de $\mathcal{D}_{m m^{\prime}}^{(j)}$ na equação $(\mathrm{A} .16 \overline{)}$ e da definição de funçōes rodadas (A.1) resulta:

$$
Y_{1 m}\left(\theta^{\prime}, \varphi^{\prime}\right)=\sum_{m^{\prime}} \mathcal{D}_{m m^{\prime}}^{1} Y_{l m^{\prime}}(-\theta, \varphi)
$$

A comparação desses dois resultados leva-nos à conclusão de que a matriz rotação para $j=1$ é a transposta de $\mathcal{M}_{s}$. Em termos de elementos ista significa que:

$$
\mathcal{D}_{m m^{\prime}}^{(1)}=\left(\mathcal{M}_{s}\right)_{m m^{\prime}}
$$

Para encontrar $\mathcal{M}_{s}$, primeiro encontramos $\mathcal{M}(\alpha \beta \gamma)$ que é o produto das três rotaçōes de Euler, $\mathcal{M}(\alpha \beta \gamma)=\mathcal{M}_{\alpha} \mathcal{M}_{\beta} \mathcal{M}_{\gamma}$, onde:

1. $\mathcal{M}(\alpha)$ - Rotação de $\alpha$ sobre $z$.

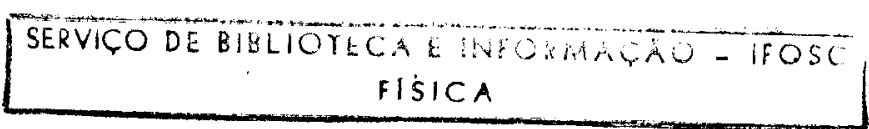


2. $\mathcal{M}(\beta)$ - Rotação de $\beta$ sobre o novo eixo $y$.

3. $\mathcal{M}(\gamma)$ - Rotação de $\gamma$ sobre o eixo $z$ final.

Calculando-se essas rotaçōes, resulta:

$\mathcal{M}(\alpha \beta \gamma)=\left(\begin{array}{ccc}\cos \alpha \cos \beta-\sin \alpha \sin \gamma & \sin \alpha \cos \beta \cos \gamma+\cos \alpha \sin \gamma & -\sin \beta \cos \gamma \\ -\cos \alpha \cos \beta \sin \gamma-\sin \alpha \cos \gamma & -\sin \alpha \cos \beta \sin \gamma+\cos \alpha \cos \gamma & \sin \beta \sin \gamma \\ \cos \alpha \sin \beta & \sin \alpha \sin \beta & \cos \beta\end{array}\right)$

A transformação unitária $\mathcal{U}$ que leva das bases cartesianas para as bases esféricas transforma o operador rotaçāo de maneira usual, pois é ela que carrega $\mathbf{r}$ em $\mathbf{r}_{\mathbf{a}}, \mathbf{r}_{\mathbf{a}}=\mathcal{U}_{\mathbf{r}}$ e $\mathbf{r}^{\prime}$ em $\mathbf{r}_{s}^{\prime}, \mathbf{r}_{s}^{\prime}=\mathcal{U} \mathbf{r}^{\prime}$. Lembrando-se de (A.21) e (A.22), onde vimos que $\mathbf{r}^{\prime}=: \mathcal{M} \vec{r}$, resulta:

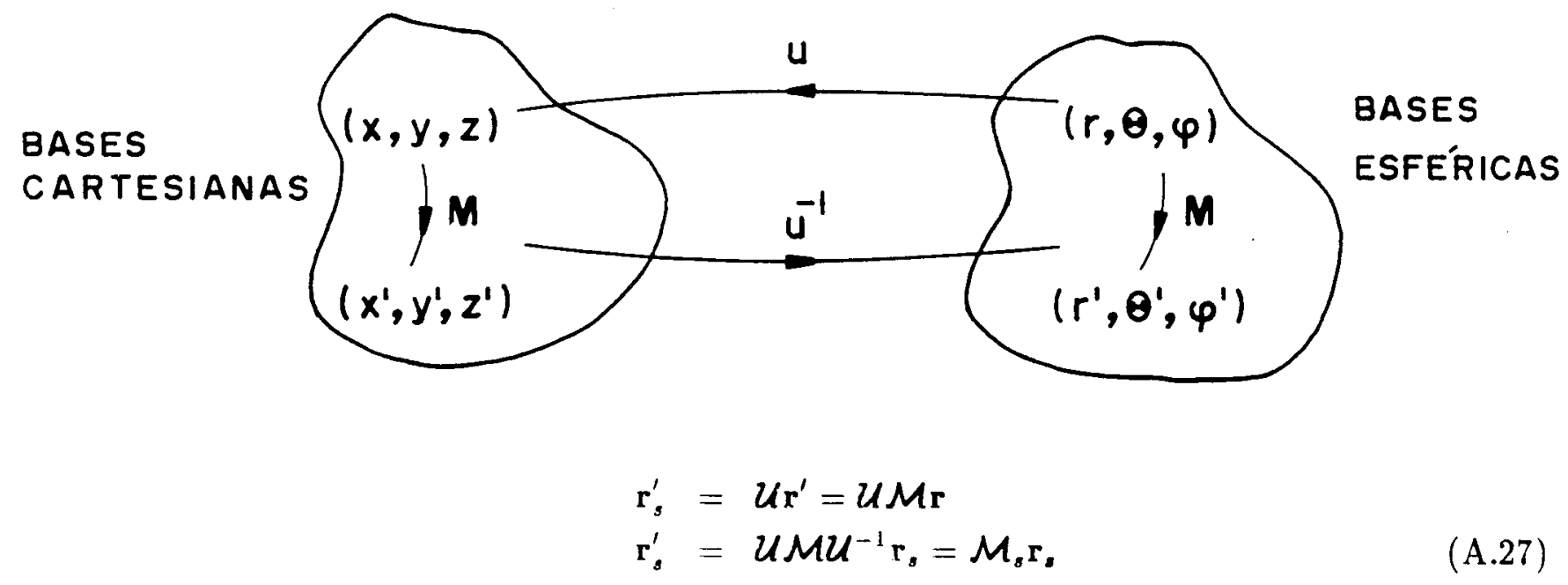

obviamente:

$$
\mathcal{M}_{s}=\mathcal{U} \mathcal{M U}^{-1}
$$

Falta ainda determinar $\mathcal{U}$. Para tal definimos de (A.20) as bases esféricas para um vetor $\mathbf{T}$ com três componesites $T_{m}(m=1,0,-1)$ :

$$
\mathbf{T}=\left(\begin{array}{l}
T_{1} \\
T_{0} \\
T_{-1}
\end{array}\right)=\left(\begin{array}{c}
-\frac{1}{\sqrt{2}}\left(T_{x}+i T_{y}\right) \\
T_{z} \\
+\frac{1}{\sqrt{2}}\left(T_{x}-i T_{y}\right)
\end{array}\right)=\mathcal{U}\left(\begin{array}{l}
T_{x} \\
T_{y} \\
T_{z}
\end{array}\right)
$$

Assim como fizemos para $\mathcal{M}$, calculamos $\mathcal{U}$ em três passos, abc $=\mathcal{U}$. sabendo-se (A.29), resulta:

$$
\mathbf{a}\left(\begin{array}{c}
x \\
y \\
z
\end{array}\right)=\left(\begin{array}{c}
x \\
i y \\
z
\end{array}\right) \Rightarrow \mathbf{a}=\left(\begin{array}{ccc}
1 & 0 & 0 \\
0 & i & 0 \\
0 & 0 & 1
\end{array}\right)
$$




$$
\begin{aligned}
\mathbf{b}\left(\begin{array}{c}
x \\
i y \\
z
\end{array}\right) & =\left(\begin{array}{c}
-\frac{1}{\sqrt{2}}(x+i y) \\
+\frac{1}{\sqrt{2}}(x-i y) \\
z
\end{array}\right) \Rightarrow \mathbf{b}=\frac{1}{\sqrt{2}}\left(\begin{array}{ccc}
-1 & -1 & 0 \\
1 & -1 & 0 \\
0 & 0 & \sqrt{2}
\end{array}\right) \\
\mathbf{c}\left(\begin{array}{c}
-\frac{1}{\sqrt{2}}(x+i y) \\
+\frac{1}{\sqrt{2}}(x-i y) \\
z
\end{array}\right) & =\left(\begin{array}{c}
-\frac{1}{\sqrt{2}}(x+i y) \\
z \\
+\frac{1}{\sqrt{2}}(x-i y)
\end{array}\right) \Rightarrow \mathbf{c}=\left(\begin{array}{lll}
1 & 0 & 0 \\
0 & 0 & 1 \\
0 & 0 & 1
\end{array}\right)
\end{aligned}
$$

De (A.30), (A.31), (A.32), resulta:

$$
U=\frac{1}{\sqrt{2}}\left(\begin{array}{ccc}
-1 & -i & 0 \\
0 & 0 & \sqrt{2} \\
1 & -i & 0
\end{array}\right)
$$

De $\mathcal{M}_{s}=U \mathcal{M U}^{-1}$ temos

$$
\mathcal{M}_{\boldsymbol{s}}(\alpha \beta \gamma)=\left(\begin{array}{ccc}
e^{-i \alpha} \frac{1+\cos \beta}{2} e^{-i \gamma} & \frac{\sin \beta}{\sqrt{2}} e^{-i \gamma} & e^{i \alpha} \frac{1-\cos \beta}{2} e^{-i \gamma} \\
\frac{\sin \beta}{\sqrt{2}} e^{-i \gamma} & \cos \beta & \frac{\sin \beta}{\sqrt{2}} e^{i \gamma} \\
e^{-i \alpha \frac{1-\cos \beta}{2}} e^{i \gamma} & -\frac{\sin \theta}{\sqrt{2}} e^{i \gamma} & e^{i \alpha} \frac{1+\cos \beta}{2} e^{i \gamma}
\end{array}\right) .
$$

A matriz rotação $\mathcal{D}^{(1)}(\alpha \beta \gamma)$, de (A.25) será

$$
\mathcal{D}^{(1)}(\alpha \beta \gamma)=\left(\begin{array}{ccc}
e^{-i \alpha \frac{1+\cos \beta}{2} e^{-i \gamma}} & -e^{-i \gamma} \frac{\sin \beta}{\sqrt{2}} & e^{-i \alpha \frac{1-\cos \beta}{2}} e^{i \gamma} \\
\frac{\sin \beta}{\sqrt{2}} e^{-i \gamma} & \cos \beta & -\frac{\sin \beta}{\sqrt{2}} e^{-i \gamma} \\
e^{i \alpha \frac{1-\cos \beta}{2} e^{-i \gamma}} & e^{i \alpha \frac{\sin \beta}{\sqrt{2}}} & e^{i \alpha \frac{1+\cos \beta}{2} e^{i \gamma}}
\end{array}\right) .
$$

A partir da Série Inversa de Clebsh-Gordon, (A.18), podemos construir as matrizes de rotação $D^{(j)}(\alpha \beta \gamma)$ para um momentum angular arbitrário $j$ que satisfaça a regra do triângulo. Resultando assim, por exemplo para $\mathcal{D}_{m^{\prime} m}^{2}(\alpha, \beta, \gamma)$, conforme apresentado na figura (A.1). 


\begin{tabular}{|c|r|r|}
\hline $\mathcal{D}_{m^{\prime} m}^{(2)}$ & $m=-1$ & $m=-2$ \\
\hline$m^{\prime}=2$ & $\frac{1-\cos \beta}{2} \sin \beta e^{i(2 \gamma-\alpha)}$ & $\frac{(1-\cos \beta)^{2}}{4} e^{2 i(\gamma-\alpha)}$ \\
\hline$m^{\prime}=1$ & {$\left[\frac{1+\cos \beta}{2}-\cos ^{2} \beta\right] e^{i(\gamma-\alpha)}$} & $\frac{1-\cos \beta}{2} \sin \beta e^{i(\gamma-2 \alpha)}$ \\
\hline$m^{\prime}=0$ & $\sqrt{\frac{3}{8}} \sin 2 \beta e^{-i \alpha}$ & $\sqrt{\frac{3}{8}} \sin ^{2} \beta e^{-2 i \alpha}$ \\
\hline$m=-1$ & {$\left[\cos ^{2} \beta-\frac{1-\cos \beta}{2}\right] e^{-i(\alpha+\gamma)}$} & $\frac{1+\cos \beta}{2} \sin \beta e^{-i(2 \alpha+\gamma)}$ \\
\hline$m^{\prime}=-2$ & $-\frac{1+\cos \beta}{2} \sin \beta e^{-i(2 \gamma+\alpha)}$ & $\frac{(1+\cos \beta)^{2}}{4} e^{-2 i(\gamma+\alpha)}$ \\
\hline
\end{tabular}

\begin{tabular}{|c|r|r|r|}
\hline $\mathcal{D}_{m^{\prime} m}^{(2)}$ & $m=2$ & $m=1$ & $m=0$ \\
\hline$m^{\prime}=2$ & $\frac{(1+\cos \beta)^{2}}{4} e^{2 i(\alpha+\gamma)}$ & $\frac{1+\cos \beta}{2} \sin \beta e^{i(2 \gamma+\alpha)}$ & $\sqrt{\frac{3}{8} \sin ^{2} \beta e^{2 i \gamma}}$ \\
\hline$m^{\prime}=1$ & $-\frac{1+\cos \beta}{2} \sin \beta e^{i(\gamma+2 \alpha)}$ & {$\left[\cos ^{2} \beta-\frac{1-\cos \beta}{2}\right] e^{i(\alpha+\gamma)}$} & $\sqrt{\frac{3}{8}} \sin 2 \beta e^{i \alpha}$ \\
\hline$m^{\prime}=0$ & $\sqrt{\frac{3}{8}} \sin ^{2} \beta e^{2 i \gamma}$ & $-\sqrt{\frac{3}{8}} \sin 2 \beta e^{i \alpha}$ & $\frac{3 \cos ^{2} \beta-1}{2}$ \\
\hline$m=-1$ & $-\frac{1-\cos \beta}{2} \sin \beta e^{i(2 \alpha-\gamma)}$ & {$\left[\frac{1+\cos \beta}{2}-\cos ^{2} \beta\right] e^{i(\alpha-\gamma)}$} & $-\sqrt{\frac{3}{8}} \sin ^{2} \beta e^{-i \gamma}$ \\
\hline$m^{\prime}=-2$ & $\frac{(1-\cos \beta)^{2}}{4} e^{2 i(\alpha-\gamma)}$ & $-\frac{1-\cos \beta}{2} \sin \beta e^{i(\alpha-2 \gamma)}$ & $\sqrt{\frac{3}{8}} \sin ^{2} \beta e^{-2 i \gamma}$ \\
\hline
\end{tabular}

Tabela A.1: Elementos da Matriz de Rotação de Wigner, $\mathcal{D}_{m^{\prime} m}^{(2)}(\alpha, \beta, \gamma)$. 


\section{Bibliografia}

[1] M. E. Rose, Elementary Theory of Angular Momentum, (1967), J. Wiley.

[2] M. Tinkham, Group Theory and Quantum Mechanics, (1964), MacGraw-Hill.

[3] R. L. Cook, F. C. De Lucia, Am.J.Phys., 39,1433 (1971).

[4] G. Goertzel, Phy.Rev.,70,897 (1946). 
BIBLIOGRAFIA 


\section{Apêndice B}

\section{Tensores Irredutiveis}

\section{B.1 Introdução}

Em nossos sistemas sempre iremos encontrar interaçōes onde a dependência de spin e coordenadas transformam-se como um tensor de segunda ordem. Como estaremos envolvidos com rotações dos Hamiltonianos de interação, nada mais conveniente que expressar esses tensores em bases esféricas.

Nessa representação esférica o operador tensor esférico de ordem $j$, é definido como um conjunto de $2 j+1$ funções $T_{j m}(m=-j,-j+1, \ldots, j)$ que transforma-se de acordo com a representação irredutível $\mathcal{D}_{m^{\prime} m}^{(j)}$, dada por:

$$
T_{j m}^{\prime}=\mathcal{R}(\alpha \beta \gamma) T_{j m} \mathcal{R}^{-1}(\alpha \beta \gamma)=\sum_{m^{\prime}=-j}^{+j} \mathcal{D}_{m^{\prime} m}^{(j)}(\alpha \beta \gamma) T_{j m^{\prime}}
$$

O operador $\mathcal{R}=e^{-i \theta \hat{n} \cdot f}$ é o operador rotação introduzido na parte A desse apêndice, sendo $\mathcal{D}_{m, m}^{(j)}(\alpha \beta \gamma)$ os elementos desse operador na representação $j m$.

Um tensor de ordem $j$ é construído a partir de dois tensores de ordem $j_{1}$ e $j_{2}$, contanto que seja satisfeita $\triangle\left(j, j_{1}, j_{2}\right)$ (Regra do Triângulo) e o número quântico projetado $m$ some-se algebricamente $\left(m=m_{1}+m_{2}\right)$, resultando:

$$
T_{j m}=\sum_{m_{1}} C\left(j_{1} j_{2} j ; m_{1}, m-m_{1}\right) V_{j_{1} m_{1}} U_{j_{2} m-m_{1}}
$$

observe que a partir de tensores $j_{1}$ e $j_{2}$ de ordem um podemos construir tensores de ordem $j$ superiores, através de (B.2).

Rose [1] provou que $T_{j m}$, definido como (B.1), é um tensor irredutível de ordem $j$ se $V_{j_{1} m_{1}}$ e $U_{j_{2} m-m_{1}}$ são componentes dum tensor irredutível de ordem $j_{1}$ e $j_{2}$ respectivamente. Quanto a ordem de um tensor resultante, essa varia de $j=\left|j_{1}+j_{2}\right|$ a $j=\left|j_{1}-j_{2}\right|$ em passos inteiros (geralmente de um a um). Disso resulta um fato importante para nossos estudos, que é a possibilidade de $j_{1}=j_{2}$, tornando-se possível construir um invariante, isto é um tensor de ordem zero. De (B.2) com $j=0, j_{1}=j_{2}$ temos: 


$$
T_{00}=\sum_{m_{1}} C\left(j_{1} j_{2} 0 ; m_{1},-m_{1}\right) V_{j_{1} m_{1}} U_{j_{2},-m_{1}}
$$

sendo $C\left(j_{1} j_{2} 0 ; m_{1},-m_{1}\right)=(-1)^{j_{1}-m_{1}}\left(2 j_{1}+1\right)^{\frac{1}{2}}$.

Observe que o termo $(-1)^{j_{1}}\left(2 j_{1}+1\right)^{\frac{1}{2}}$ pode se retirado para fora da soma e ser absorvido por $T_{00}$, resultando:

$$
\Im_{j}=\sum_{m}(-1)^{m} V_{j m} U_{j,-m}
$$

que é um invariante. Usaremos-nos desse fato quando tratarmos dos nossos Hamiltonianos de interação.

\section{B.2 Componentes de um Tensor Irredutível $\ell=2$}

É conveniente agora expressarmos as componentes de um tensor irredutível de segunda ordem tanto em coordenadas cartesianas como esféricas. Para tal iniciamos com um tensor de ordem um, designado por $\mathbf{T}_{1}$, onde suas componentes esféricas $T_{1 m}(m=1,0,-1)$ são dadas por:

$$
\begin{aligned}
T_{1 m} & =\hat{\mathbf{e}}_{1 m} \cdot \mathbf{T} \\
& =\hat{\mathbf{e}}_{1 m} \cdot\left(T_{x} \hat{\mathbf{x}}+T_{y} \hat{\mathbf{y}}+T_{z} \hat{\mathbf{z}}\right)
\end{aligned}
$$

Vimos que para um tensor de ordem um, suas bases são dadas pelo harmônico esférico de ordem um, conforme (A.20), resultando para as bases esféricas $\hat{\mathbf{e}}_{1 m}$ :

$$
\begin{aligned}
\hat{\mathbf{e}}_{10} & =\hat{\mathbf{e}}_{z}, \\
\hat{\mathbf{e}}_{1 \pm 1} & =\mp \frac{\hat{\mathbf{e}}_{x}-i \hat{\mathbf{e}}_{y}}{\sqrt{2}} \equiv \frac{\hat{\mathbf{e}}_{ \pm}}{\sqrt{2}}
\end{aligned}
$$

onde $\hat{\mathbf{e}}_{ \pm}$são vetores unitário de rotação sendo \pm a direção dessa rotação.

Resulta assim para as componentes de um tensor irredutível de ordem um (B.5) em termos das componentes cartesianas:

$$
\begin{aligned}
T_{01} & =T_{z} \\
T_{1 \pm 1} & =\mp\left(\frac{1}{\sqrt{2}}\left(T_{x} \pm i T_{y}\right) .\right.
\end{aligned}
$$

Agora para um tensor irredutível de ordem dois $(j=2)$, com $j_{1}=j_{2}=1$, obtemos-o com a contração desses dois tensores de ordem um, $V_{1} \& U_{1}$, conforme (B.2), resultando:

$$
T_{j m}=\sum_{m_{1}} C\left(11 j ; m_{1}, m-m_{1}\right) V_{1 m_{1}} U_{1 m-m_{1}}
$$


substituindo os coeficientes de Clabsh-Gordon resulta para os elementos:

$$
\begin{aligned}
T_{00} & =\frac{1}{\sqrt{3}}\left(U_{11} V_{1-1}-U_{10} V_{10}+U_{1-1} V_{11}\right) \\
T_{11} & =\frac{1}{\sqrt{2}}\left(U_{11} V_{10}-U_{10} V_{11}\right) \\
T_{10} & =\frac{1}{\sqrt{2}}\left(U_{11} V_{1-1}-U_{1-1} V_{11}\right) \\
T_{1-1} & =\frac{1}{\sqrt{2}}\left(-U_{1-1} V_{10}+U_{10} V_{1-1}\right) \\
T_{22} & =U_{11} V_{11}, \\
T_{21} & =\frac{1}{\sqrt{2}}\left(U_{11} V_{10}+U_{10} V_{11}\right) \\
T_{20} & =\frac{1}{\sqrt{6}}\left(2 U_{10} V_{10}+U_{11} V_{1-1}+U_{1-1} V_{11}\right), \\
T_{2-1} & =\frac{1}{\sqrt{2}}\left(U_{1-1} V_{10}+U_{10} V_{1-1}\right), \\
T_{2-2} & =U_{1-1} V_{1-1} .
\end{aligned}
$$

Agora utilizando-se de (B.8), resulta para $T_{j m}$ em termos das coordenadas cartesianas:

$$
\begin{aligned}
T_{00} & =-\frac{1}{3}\left(T_{x x}+T_{y y}+T_{z z}\right), \\
T_{10} & =-\frac{i}{\sqrt{2}}\left(T_{x y}-T_{y x}\right), \\
T_{1 \pm 1} & =-\frac{1}{2}\left[T_{z x}-T_{x z} \pm i\left(T_{z y}-T_{y z}\right)\right], \\
T_{20} & =\frac{1}{\sqrt{6}}\left[3 T_{z z}-\left(T_{x x}+T_{y y}+T_{z z}\right)\right], \\
T_{2 \pm 1} & =\mp \frac{1}{2}\left[T_{x z}+T_{z x} \pm i\left(T_{y z}+T_{z y}\right)\right], \\
T_{2 \pm 2} & =\frac{1}{2}\left[T_{x x}-T_{y y} \pm i\left(T_{x y}+T_{y x}\right)\right] .
\end{aligned}
$$

Observe que um tensor cartesiano de segunda ordem é decomposto em três tensores irredutíveis de ordem 0,1 e 2 :

$$
\mathbf{T}=\mathbf{T}^{0}+\mathbf{T}^{1}+\mathbf{T}^{2}
$$

É aparente também de (B.9) que num caso de tensor cartesiano simétrico e de traço nulo, somente o tensor irredutível de ordem 2 não se anulam. 


\section{Bibliografia}

[1] M. E. Rose, Elementary Theory of Angular Momentum, (1967), J. Wiley.

[2] M. Tinkham, Group Theory and Quantum Mechanics, (1964), McGraw-Hill.

[3] R. L. Cook, F. C. De Lucia, Am.J.Phys., 39, 1433 (1971). 
BIBLIOGRAFIA 


\section{Apêndice $\mathrm{C}$}

\section{Projeto do Sistema de Rotação da Amostra}

Neste apêndice estão reunidos os desenhos do projeto do sistema de rotação da amostra desenvolvido na oficina do DFCM-IFQSC-USP, na seguinte ordem:

1. Aspecto geral da montagem do sistema de rotação sobre o suporte de sustentação. Também são apresentados o sistema de engrenagem para o posicionamento do rotor no ângulo mágico e detalhe em perspectiva do sistema de rotação (detalhe $\mathrm{A}$ ).

2. Vista de elevação e planta em corte (AB) do sistema de rotação.

3. Apresentação do Detalhe A do item 2.

4. Detalhes e dimensões do "stator", com a bobina de RF posicionada.

5. Detalhes e dimensōes do rotor e sua tampa ("end caps") com aletas.

6. Detalhes e dimensões do "housing".

7. Detalhes e dimensões do suporte do sistema de rotação. 
CIRCUITO DE

ACOPLAMENTO

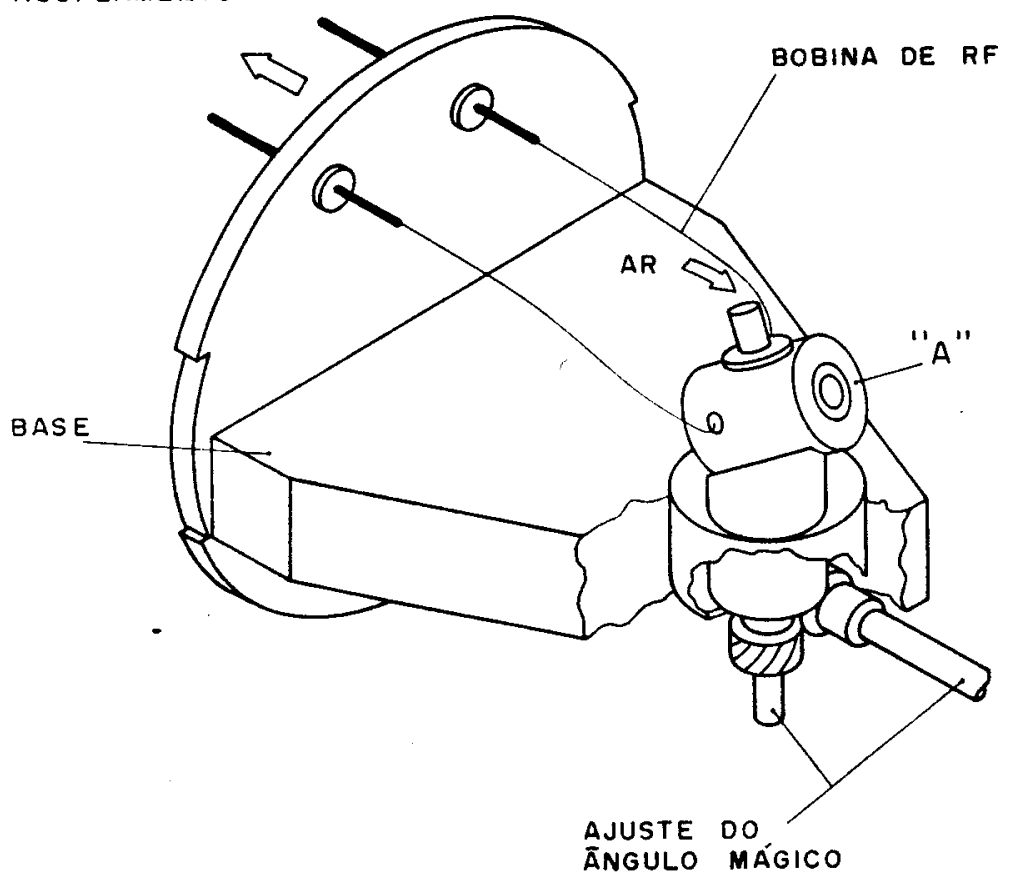

ÁNGULO MÁGICO
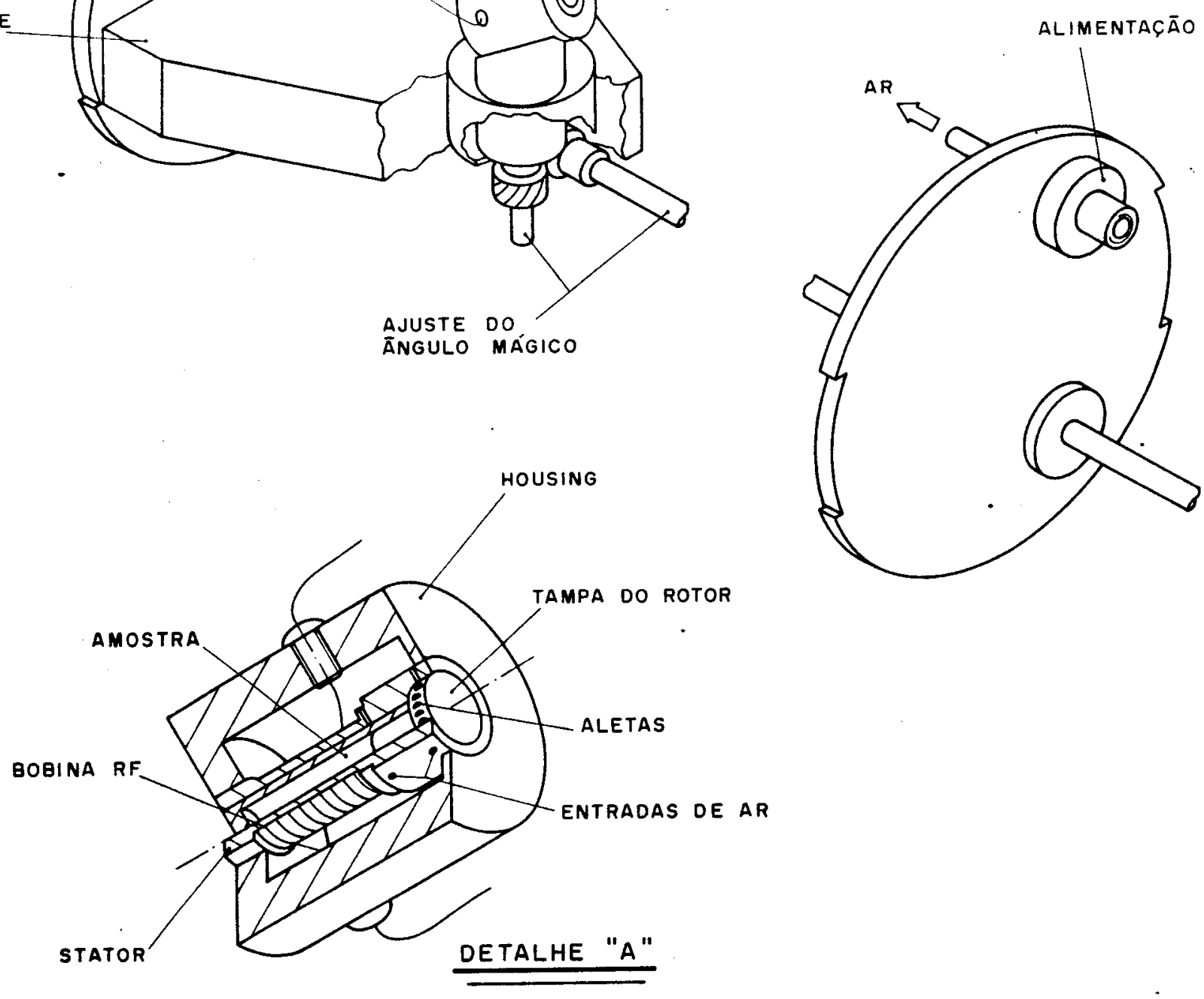

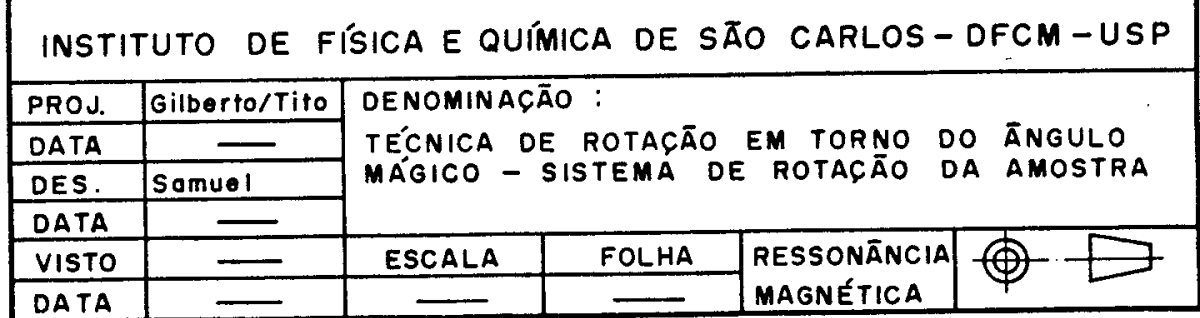




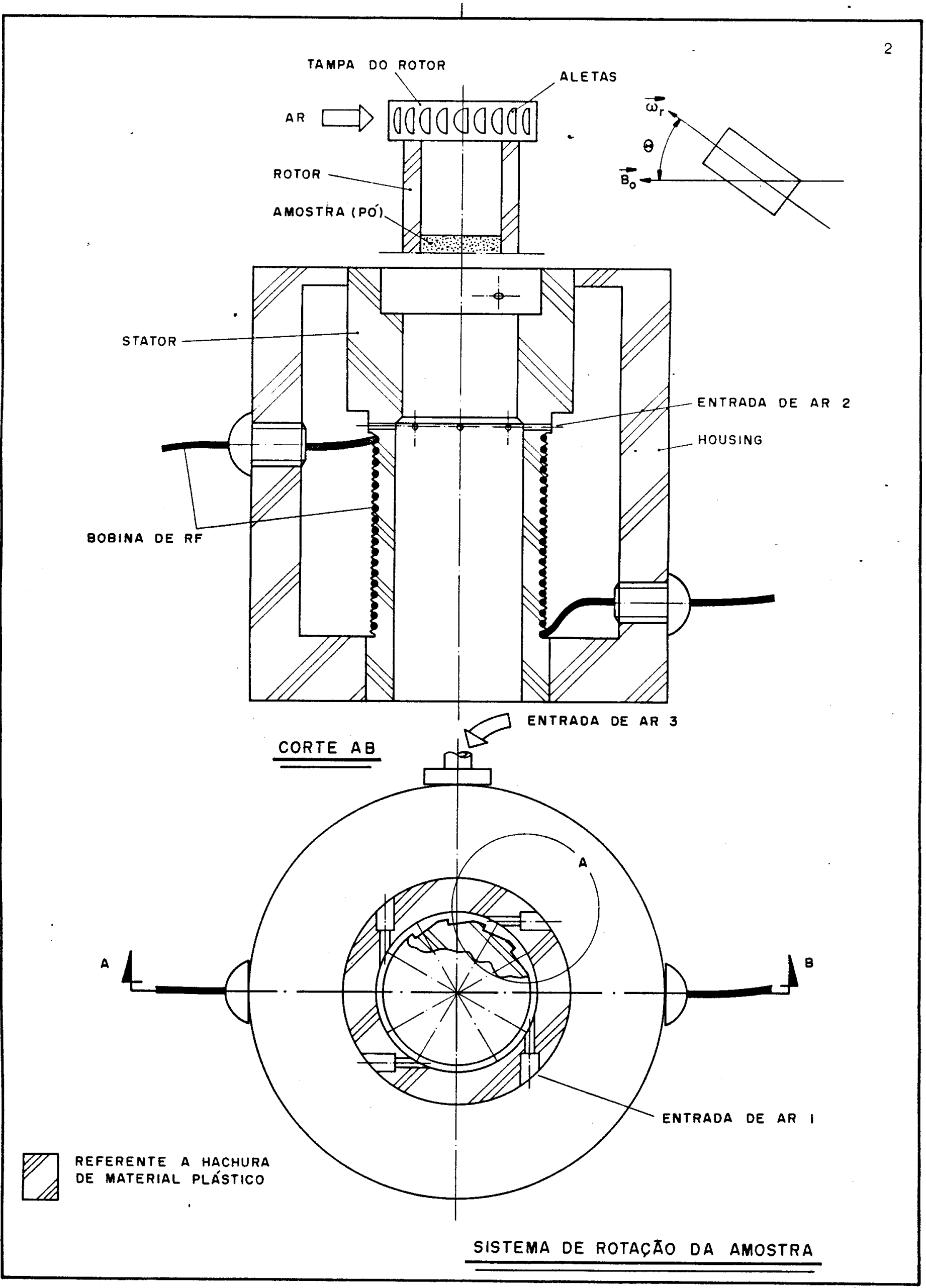




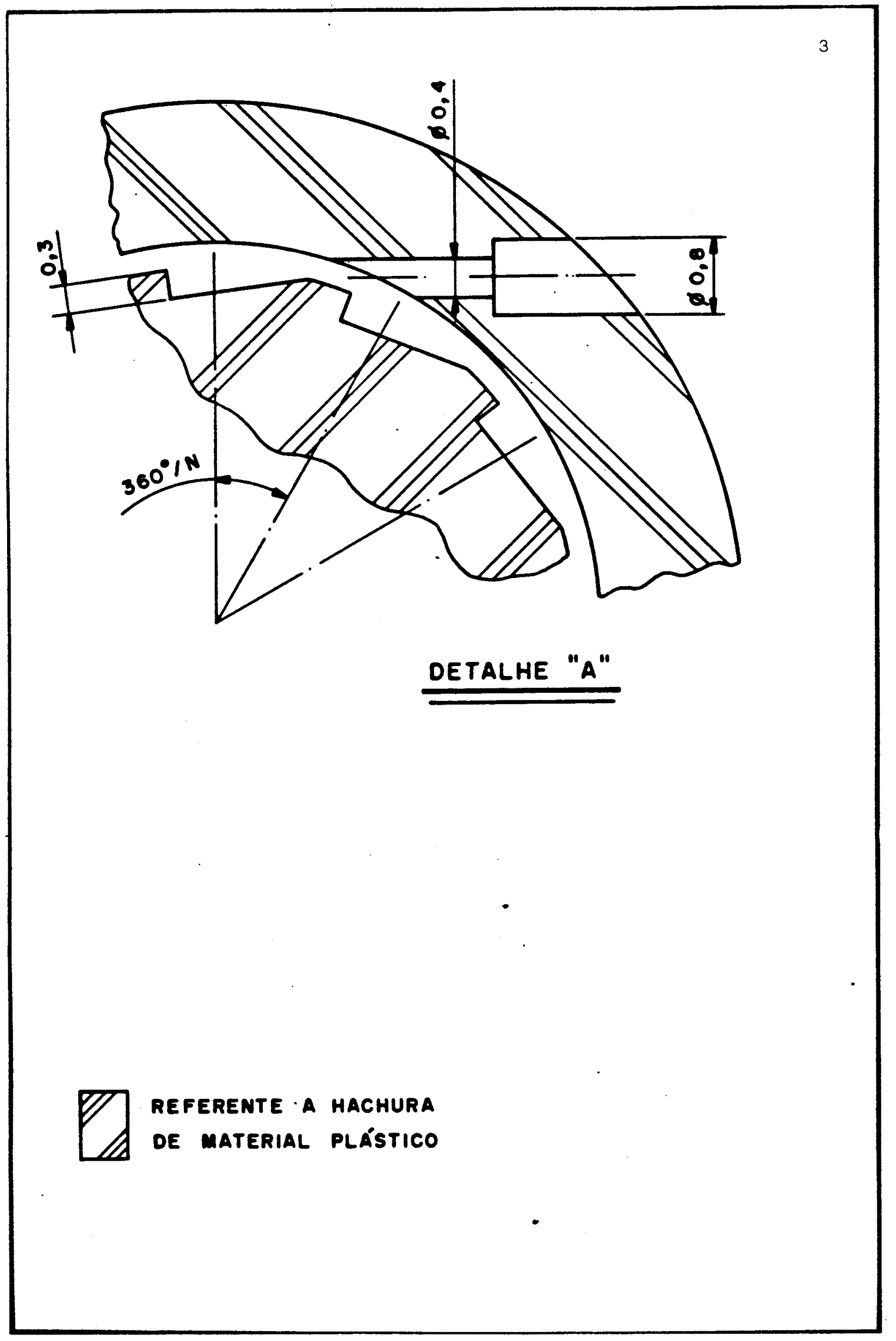




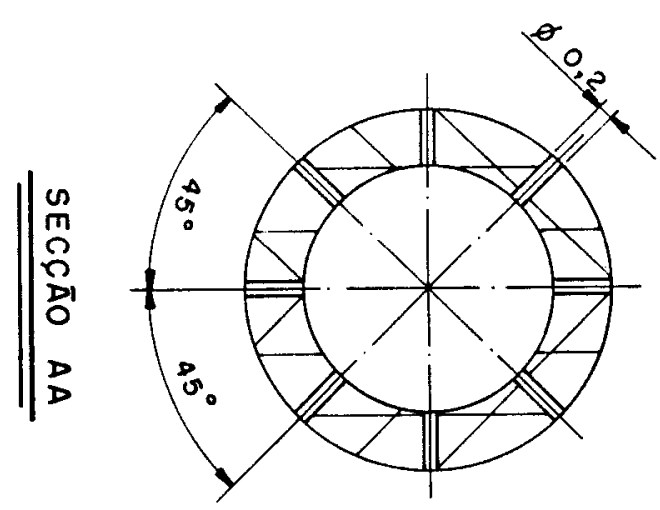

$\|^{0}=$
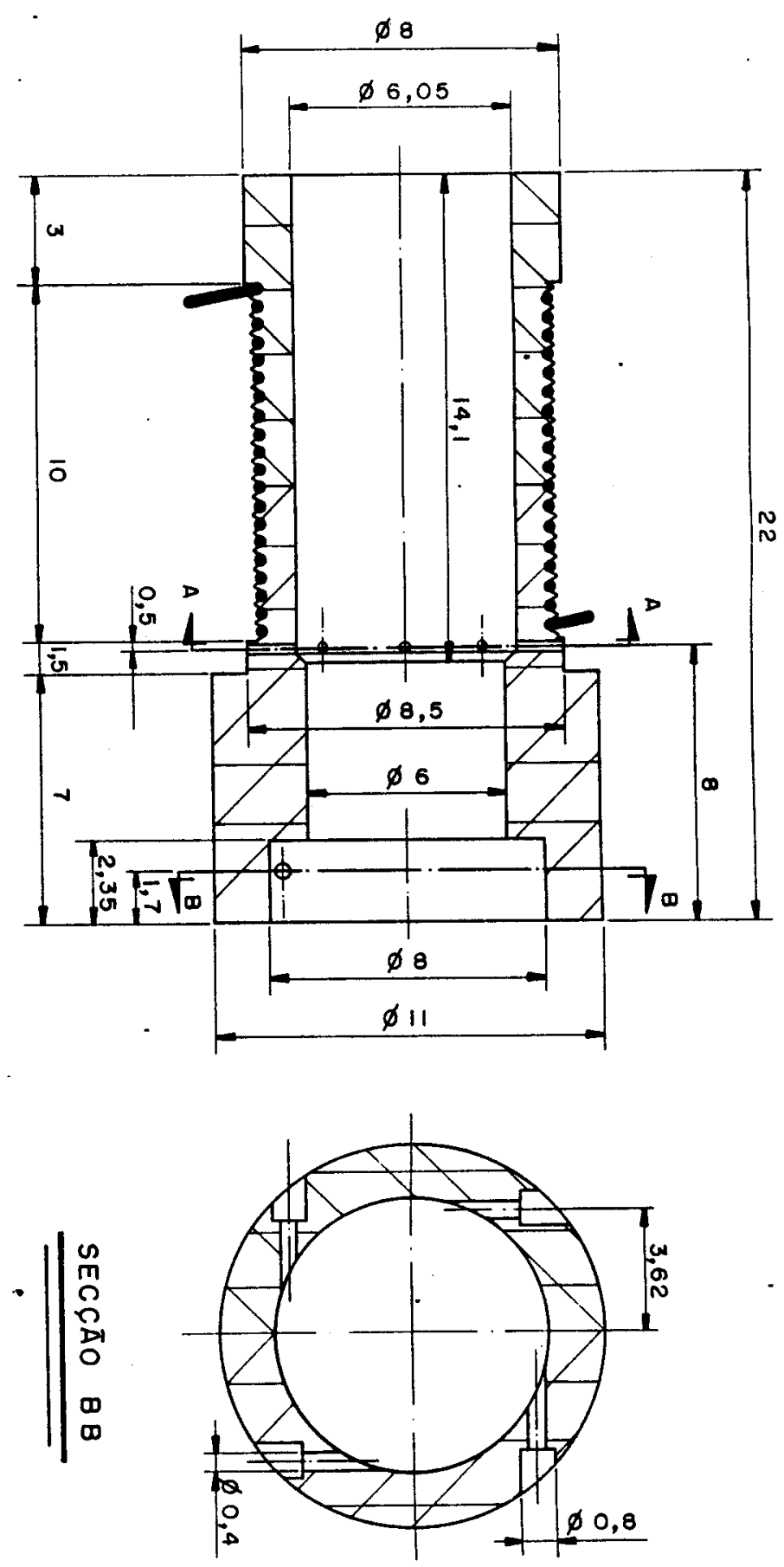
N aletas

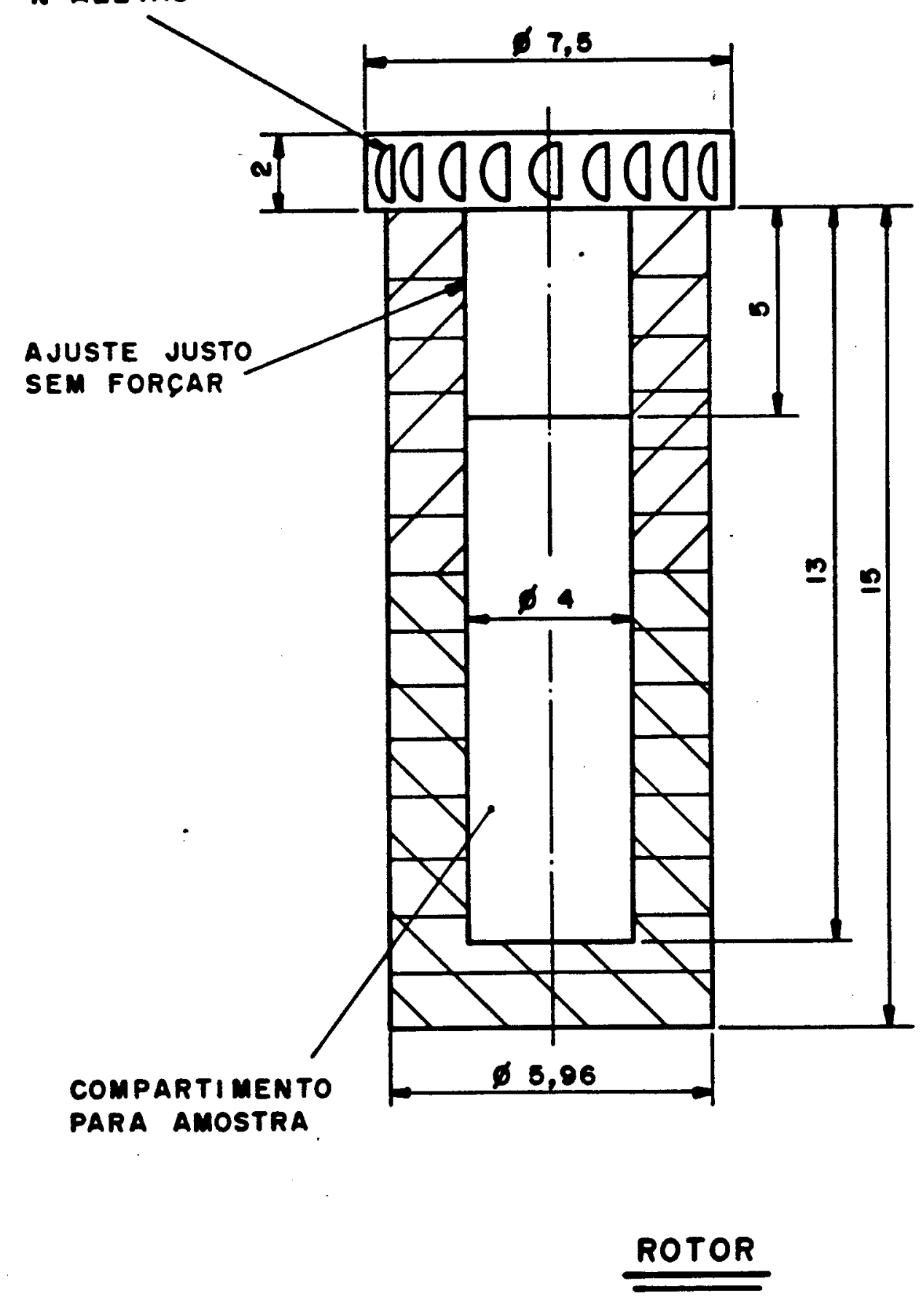




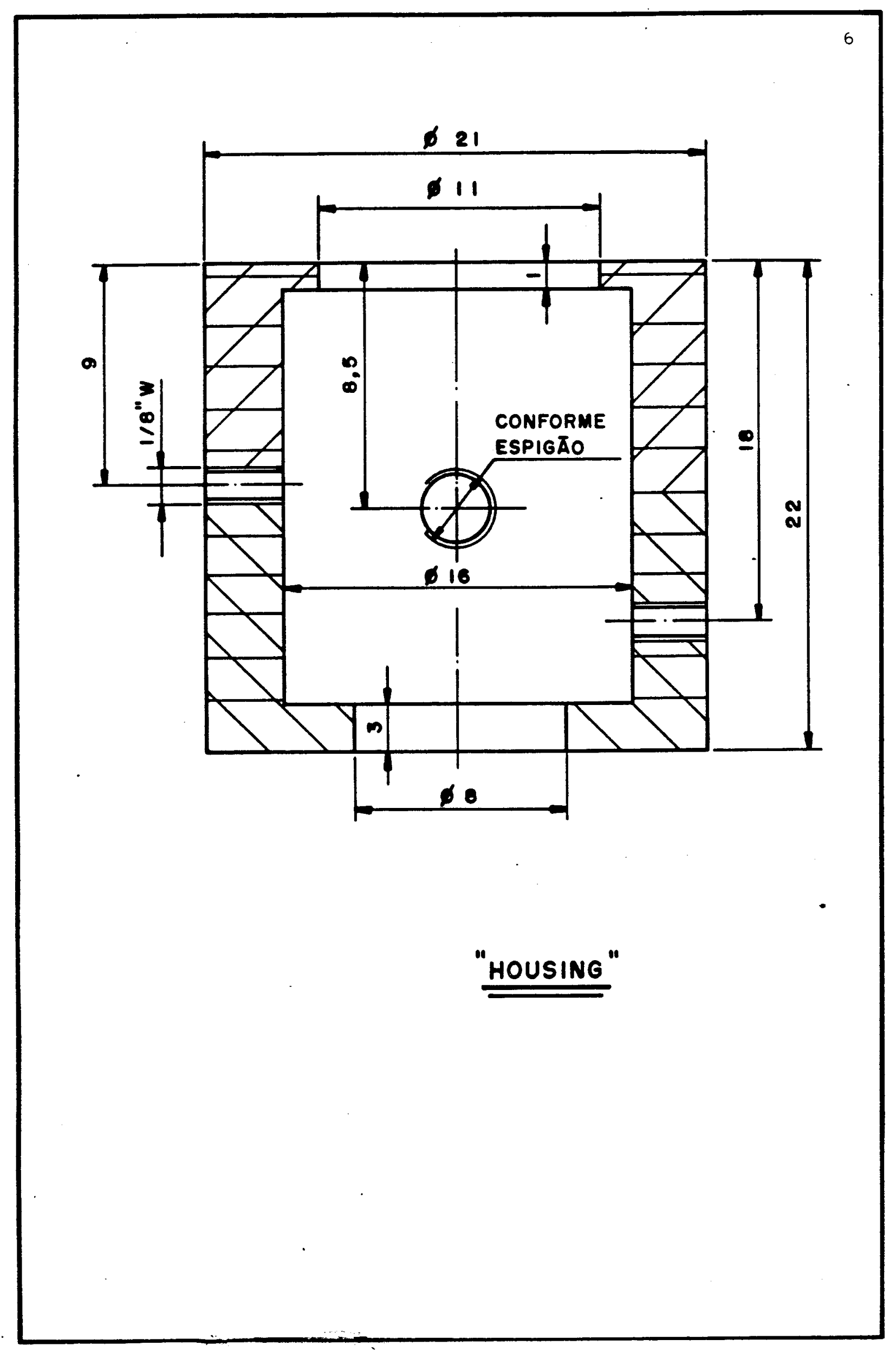

SERVICO DE BIULIOUECA E INFORMACXO-IFOSC fISICA 


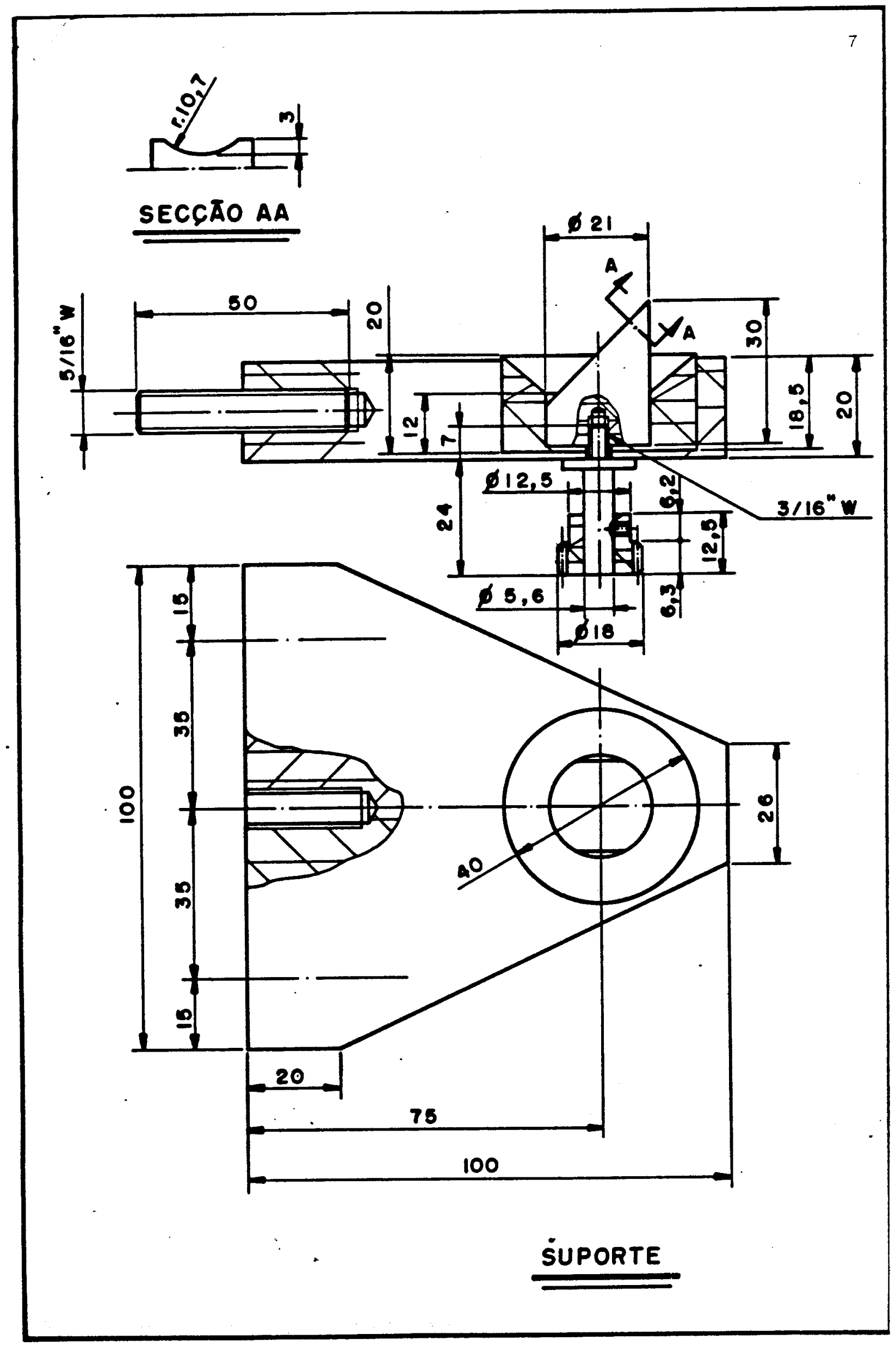

